Cochrane Database of Systematic Reviews

\title{
Parent training interventions for Attention Deficit Hyperactivity Disorder (ADHD) in children aged 5 to 18 years (Review)
}

Zwi M, Jones H, Thorgaard C, York A, Dennis JA

Zwi M, Jones H, Thorgaard C, York A, Dennis JA.

Parent training interventions for Attention Deficit Hyperactivity Disorder (ADHD) in children aged 5 to 18 years.

Cochrane Database of Systematic Reviews 2011, Issue 12. Art. No.: CD003018.

DOI: 10.1002/14651858.CD003018.pub3.

www.cochranelibrary.com

Parent training interventions for Attention Deficit Hyperactivity Disorder (ADHD) in children aged 5 to 18 years (Review) Copyright @ 2011 The Cochrane Collaboration. Published by John Wiley \& Sons, Ltd. 


\section{TABLE OF CONTENTS}

HEADER

ABSTRACT

PLAIN LANGUAGE SUMMARY

BACKGROUND

OBJECTIVES

METHODS

RESULTS

Figure 1.

Figure 2.

DISCUSSION

AUTHORS' CONCLUSIONS

ACKNOWLEDGEMENTS

REFERENCES

CHARACTERISTICS OF STUDIES

DATA AND ANALYSES

Analysis 1.1. Comparison 1 Parent training versus control, Outcome 11 Child's ADHD behaviour (home setting) CPRS-

R.S.

Analysis 1.2. Comparison 1 Parent training versus control, Outcome 2 2a Externalising.

Analysis 1.3. Comparison 1 Parent training versus control, Outcome $32 \mathrm{~b}$ Internalising.

Analysis 1.4. Comparison 1 Parent training versus control, Outcome 47 Parenting stress - PSI - parent domain.

Analysis 1.5. Comparison 1 Parent training versus control, Outcome 57 Parenting stress PSI - child domain. . . APPENDICES

HISTORY

CONTRIBUTIONS OF AUTHORS

DECLARATIONS OF INTEREST

SOURCES OF SUPPORT

DIFFERENCES BETWEEN PROTOCOL AND REVIEW

NOTES

INDEX TERMS 


\title{
[Intervention Review]
}

\section{Parent training interventions for Attention Deficit Hyperactivity Disorder (ADHD) in children aged 5 to 18 years}

\author{
Morris Zwi ${ }^{1}$, Hannah Jones ${ }^{2}$, Camilla Thorgaard ${ }^{3}$, Ann York ${ }^{1}$, Jane A Dennis ${ }^{4}$ \\ ${ }^{1}$ Richmond Royal Hospital, South West London \& St George's NHS Mental Health Trust, Richmond, UK. ${ }^{2}$ Cochrane Schizophrenia \\ Group, University of Nottingham, Nottingham, UK. ${ }^{3}$ Kraftens Bekæmpelse, Strandboulevarden 49, København $\varnothing$, Denmark. ${ }^{4} \mathrm{c} / \mathrm{o}$ \\ Developmental, Psychosocial and Learning Problems Group, Queen's University, Belfast, UK
}

Contact address: Morris Zwi, Richmond Royal Hospital, South West London \& St George's NHS Mental Health Trust, Kew Foot Road, Richmond, Surrey, TW9 2TE, UK. mzwi@btinternet.com, morris.zwi@swlstg-tr.nhs.uk.

Editorial group: Cochrane Developmental, Psychosocial and Learning Problems Group.

Publication status and date: New, published in Issue 12, 2011.

Citation: Zwi M, Jones H, Thorgaard C, York A, Dennis JA. Parent training interventions for Attention Deficit Hyperactivity Disorder (ADHD) in children aged 5 to 18 years. Cochrane Database of Systematic Reviews 2011, Issue 12. Art. No.: CD003018. DOI: 10.1002/14651858.CD003018.pub3.

Copyright (C) 2011 The Cochrane Collaboration. Published by John Wiley \& Sons, Ltd.

\begin{abstract}
A B S T R A C T
Background

Attention Deficit Hyperactivity Disorder (ADHD) is a neurodevelopmental disorder characterised by high levels of inattention, hyperactivity and impulsivity that are present before the age of seven years, seen in a range of situations, inconsistent with the child's developmental level and causing social or academic impairment. Parent training programmes are psychosocial interventions aimed at training parents in techniques to enable them to manage their children's challenging behaviour.
\end{abstract}

\section{Objectives}

To determine whether parent training interventions are effective in reducing ADHD symptoms and associated problems in children aged between five and eigtheen years with a diagnosis of ADHD, compared to controls with no parent training intervention.

\section{Search methods}

We searched the following electronic databases (for all available years until September 2010): CENTRAL (2010, Issue 3), MEDLINE (1950 to 10 September 2010), EMBASE (1980 to 2010 Week 36), CINAHL (1937 to 13 September 2010), PsycINFO (1806 to September Week 1 2010), Dissertation Abstracts International (14 September 2010) and the metaRegister of Controlled Trials (14 September 2010). We contacted experts in the field to ask for details of unpublished or ongoing research.

\section{Selection criteria}

Randomised (including quasi-randomised) studies comparing parent training with no treatment, a waiting list or treatment as usual (adjunctive or otherwise). We included studies if ADHD was the main focus of the trial and participants were over five years old and had a clinical diagnosis of ADHD or hyperkinetic disorder that was made by a specialist using the operationalised diagnostic criteria of the DSM-III/DSM-IV or ICD-10. We only included trials that reported at least one child outcome.

\section{Data collection and analysis}

Four authors were involved in screening abstracts and at least 2 authors looked independently at each one. We reviewed a total of 12,691 studies and assessed five as eligible for inclusion. We extracted data and assessed the risk of bias in the five included trials. Opportunities for meta-analysis were limited and most data that we have reported are based on single studies.

Parent training interventions for Attention Deficit Hyperactivity Disorder (ADHD) in children aged 5 to 18 years (Review)

Copyright () 20 I I The Cochrane Collaboration. Published by John Wiley \& Sons, Ltd. 


\section{Main results}

We found five studies including 284 participants that met the inclusion criteria, all of which compared parent training with de facto treatment as usual (TAU). One study included a nondirective parent support group as a second control arm.

Four studies targeted children's behaviour problems and one assessed changes in parenting skills.

Of the four studies targeting children's behaviour, two focused on behaviour at home and two focused on behaviour at school. The two studies focusing on behaviour at home had different findings: one found no difference between parent training and treatment as usual, whilst the other reported statistically significant results for parent training versus control. The two studies of behaviour at school also had different findings: one study found no difference between groups, whilst the other reported positive results for parent training when ADHD was not comorbid with oppositional defiant disorder. In this latter study, outcomes were better for girls and for children on medication.

We assessed the risk of bias in most of the studies as unclear at best and often as high. Information on randomisation and allocation concealment did not appear in any study report. Inevitably, blinding of participants or personnel was impossible for this intervention; likewise, blinding of outcome assessors (who were most often the parents who had delivered the intervention) was impossible.

We were only able to conduct meta-analysis for two outcomes: child 'externalising' behaviour (a measure of rulebreaking, oppositional behaviour or aggression) and child 'internalising' behaviour (for example, withdrawal and anxiety). Meta-analysis of three studies ( $\mathrm{n}=$ 190) providing data on externalising behaviour produced results that fell short of statistical significance (SMD - $0.32 ; 95 \%$ CI -0.83 to $\left.0.18, \mathrm{I}^{2}=60 \%\right)$. A meta-analysis of two studies $(\mathrm{n}=142)$ for internalising behaviour gave significant results in the parent training groups (SMD - $0.48 ; 95 \%$ CI -0.84 to $-0.13, \mathrm{I}^{2}=9 \%$ ). Data from a third study likely to have contributed to this outcome were missing, and we have some concerns about selective outcome reporting bias.

Individual study results for child behaviour outcomes were mixed. Positive results on an inventory of child behaviour problems were reported for one small study $(\mathrm{n}=24)$ with the caveat that results were only positive when parent training was delivered to individuals and not groups. In another study $(n=62)$, positive effects (once results were adjusted for demographic and baseline data) were reported for the intervention group on a social skills measure.

The study $(\mathrm{n}=48)$ that assessed parenting skill changes compared parent training with a nondirective parent support group. Statistically significant improvements were reported for the parent training group. Two studies $(\mathrm{n}=142)$ provided data on parent stress indices that were suitable for combining in a meta-analysis. The results were significant for the 'child' domain (MD - 10.52; $95 \%$ CI -20.55 to 0.48 ) but not the 'parent' domain (MD -7.54; 95\% CI -24.38 to 9.30). Results for this outcome from a small study ( $\mathrm{n}=24)$ suggested a long-term benefit for mothers who received the intervention at an individual level; in contrast, fathers benefited from short-term group treatment. A fourth study reported change data for within group measures of parental stress and found significant benefits in only one of the two active parent training group arms $(\mathrm{P} \leq 0.01)$.

No study reported data for academic achievement, adverse events or parental understanding of ADHD.

\section{Authors' conclusions}

Parent training may have a positive effect on the behaviour of children with ADHD. It may also reduce parental stress and enhance parental confidence. However, the poor methodological quality of the included studies increases the risk of bias in the results. Data concerning ADHD-specific behaviour are ambiguous. For many important outcomes, including school achievement and adverse effects, data are lacking.

Evidence from this review is not strong enough to form a basis for clinical practice guidelines. Future research should ensure better reporting of the study procedures and results.

\section{PLAIN LANGUAGE SUMMARY}

\section{Parent training for Attention Deficit Hyperactivity Disorder (ADHD) in children aged 5 to 18 years}

Attention Deficit Hyperactivity Disorder (ADHD) is a neurodevelopmental disorder. For a child to be diagnosed with ADHD, adults such as parents, carers, healthcare workers or teachers must have noticed higher levels of inattention, hyperactivity and impulsivity in the child before the age of seven years compared to children of similar age. The inattention, hyperactivity and impulsivity must be observed in a range of situations, for a substantial period of time and cause impairment to the child's learning or social development.

Parent training interventions for Attention Deficit Hyperactivity Disorder (ADHD) in children aged 5 to 18 years (Review)

Copyright ( 201 I The Cochrane Collaboration. Published by John Wiley \& Sons, Ltd. 
Parent training programmes aim to equip parents with techniques to manage their child's 'difficult' or ADHD-related behaviour (that is their inattention and hyperactivity-impulsivity).

We found five randomised controlled studies that met our inclusion criteria. Four set out to improve children's general behaviour and one focused specifically on how parents could help their children make friends. All studies were small and their quality varied. Results from these studies were somewhat encouraging as far as parental stress and general child behaviour were concerned, but were uncertain with regard to other important outcomes including ADHD-related behaviour. No study provided data on the key outcomes of achievement in school, harmful effects or parent knowledge of ADHD. There was no evidence to say whether parent training is better delivered in groups or individually.

The evidence we found was limited in terms of the size of the trials and in their quality, and therefore we do not think it can be used as the basis for guidelines of treatment of ADHD in clinics or schools. We believe more research is needed and that it should ensure better reporting of the study procedures and results.

\section{B A C K G R O U N D}

\section{Description of the condition}

\section{Definition of Attention Deficit Hyperactivity Disorder (ADHD) and its prevalence}

ADHD is a neurodevelopmental disorder characterised by high levels of inattention, hyperactivity and impulsivity that are present before seven years of age. These are seen in a range of situations, are inconsistent with the developmental level of the child and are associated with impairment in social or academic development (APA 1994). In the International Classification of Diseases (ICD-10) (WHO 1992), hyperkinetic disorder (HKD) is similar to ADHD but the criteria are more restrictive. In this review we used the term ADHD to include $\mathrm{HKD}$, although technically $\mathrm{HKD}$ defines a more severe subgroup of ADHD (WHO 1992; APA 1994). Prevalence estimates for ADHD vary considerably and depend on the characteristics of the population, sampling methods and the nature of the assessment (Jadad 1999a; Faraone 2003; Sciutto 2007). In a UK survey of 10,438 children aged five to 15 years, Ford 2003 found that $3.62 \%$ of boys and $0.85 \%$ of girls had a diagnosis of ADHD. The Center for Disease Control and Prevention in the USA conducted a survey of 102,353 parents with children aged four to 17 years and found a lifetime childhood diagnosis of $7.8 \%$ (2.5 times as many boys as girls) of whom $4.3 \%$ had received medication as treatment (CDC 2005). A review of 50 prevalence studies (including 20 US and 30 non-US sample populations) suggested that the prevalence is similar in US and non-US populations (Faraone 2003). However, the 'administrative' prevalence, that is the frequency of diagnosis in practice, seems to highlight a cultural difference between US and European clinicians as this ratio is estimated to be as high as 20:1 (Santosh 2005).

Comorbidity between ADHD and conduct problems is high. In the British Child and Adolescent Mental Health Survey, 27\% of those with conduct disorder (CD) and $26 \%$ of those with oppositional defiant disorder (ODD) also qualified for a diagnosis of $\mathrm{ADHD}$, and more than $50 \%$ of those with ADHD had a comorbid behaviour disorder (Ford 2003).

Early conduct problems appear to precede antisocial behaviour in later life. Farrington 1995 estimated that it is possible to predict over half of future recidivist delinquents based on their aggressive behaviour and a family's ineffective child rearing practices. The precise relationship between conduct disorder or oppositional defiant disorder and ADHD, especially the mechanism of development of antisocial behaviour in children with ADHD, is not, however, fully understood.

\section{Aetiology}

It is thought that genetic and environmental risk factors interact to cause ADHD rather than operate in isolation (Pliszka 2007; NICE 2008). The genetic contribution to observable phenotypic ADHD traits has been estimated as being up to $76 \%$ (Faraone 2005). No large single gene effect has been identified but the DRD4 and DRD5 genes appear to be involved (Li 2006) and a specific haplotype of the dopamine transporter gene has also been associated with the combined-type ADHD (Asherson 2007). Findings by Williams 2010 indicated an increased rate of chromosomal deletions and duplications in children with ADHD compared to those without ADHD, suggesting further evidence of genetic influence in ADHD development. Controversial and inaccurate press reporting of this study highlights the need for clarity in communication of complex scientific work by journalists (Jones 2010; McFadden 2010). 
Possible environmental factors include maternal smoking, alcohol consumption, heroin use in pregnancy, foetal hypoxia and perinatal exposure to toxins, injury and zinc deficiency (NICE 2008).

\section{Issues regarding diagnosis}

The diagnosis of ADHD has stimulated considerable debate and sometimes strong and conflicting views (Jadad 1999b). Inattention, hyperactivity and impulsivity are normal traits in children, especially young children. There is no reliable test to confirm diagnostic validity, so diagnoses depend on clinical judgment. Consensus among experts in the field (Barkley 2002a) might exist but diagnoses may be open to bias. The use of operationalised diagnostic criteria, such as the DSM-IV (APA 1994) or ICD-10 (WHO 1992), may reduce such bias. Professional and national bodies that are concerned about the importance of thorough and accurate ADHD diagnoses have issued guidelines to encourage good practice (American Academy of Pediatrics 2001; SIGN 2009; Pliszka 2007; NICE 2008).

Preschool diagnosis of ADHD is particularly problematic because few data on preschool diagnostic practice exist (Sonuga-Barke 2003a). Lahey 2004 demonstrated that many four to six year olds continue to meet ADHD diagnostic criteria three years on; however factors such as parental expectations might influence the assessment of the severity of the condition and the extent of impairment it causes. Even where particular symptom levels are judged to be out of the normal range for that age, this might be transitory and reflect normal step-wise or non-linear competency development (Sonuga-Barke 2003a). It is a complex process to establish 'caseness' (meeting the criteria for ADHD) where high levels of inattention and hyperactivity exist in a preschool child. The unusual extent of these traits and their association with impairment in the particular child must be demonstrated in order to meet DSM-IV diagnostic criteria (APA 1994). Because it is uncertain that this can be done in a systematic and precise way, it has been suggested that making consistent diagnostic assessments in this age group could be problematic (Sonuga-Barke 2003a).

Concerns about diagnostic validity led to the Preschool ADHD Treatment Study (PATS) (Kollins 2006). Kollins 2006 demonstrated that when parent- and teacher-rated ADHD symptoms are examined, DSM-IV symptoms do not consistently act as meaningful discriminators in identifying ADHD and its subtypes; "it may be that other symptoms not routinely assessed in this age group are more saliently associated with ADHD in the preschool years" (Hardy 2007).

Our review requires that trial participants have a valid ADHD diagnosis. In view of the concerns about diagnostic validity of $\mathrm{ADHD}$ in preschool children 'at risk' of developing ADHD, we decided that this group fell outside the scope of our review. Without definite ADHD diagnoses, uncertainty would exist as to whether any behavioural and symptom changes with the intervention occurred in children truly at risk of developing ADHD, as opposed to those with opposition defiant disorder or conduct disorder or simply going through a particular developmental stage.

\section{Treatment}

\section{Pharmacological}

Over the past decade, a number of systematic reviews on ADHD treatment have been published (Miller 1998; Jadad 1999a; NICE 2000; SIGN 2009; NICE 2006b; NICE 2008). They conclude that stimulant treatments are relatively safe and effective (at least in the short term) for managing the ADHD core symptoms, which are excessive inattention, hyperactivity and impulsivity. Atomoxetine, a noradrenergic reuptake inhibitor, has also been found to be effective (Michelson 2001; NICE 2006b; Cheng 2007). Professional guidelines recommend pharmacological treatments, with or without concomitant psychosocial interventions, after a comprehensive diagnostic assessment (American Academy of Pediatrics 2001; Taylor 2004; Pliszka 2007; NICE 2008). Whilst consistent treatment with stimulants is associated with maintenance of effectiveness, it is also associated with 'mild' suppression of growth (MTA 2004).

Adding psychological interventions to medication has not been demonstrated to improve outcomes significantly (Miller 1998; MTA 1999; Abikoff 2004). However, it has been argued that stimulants do not necessarily lead to long-term benefits (Jensen 2007). Those children with more severe symptoms appear to do better with stimulants than with behavioural interventions (Santosh 2005) but subgroups of ADHD patients with comorbid disorders might respond differentially to pharmacological and psychological treatments (Jensen 2001).

\section{Psychosocial}

Over recent years there has been an increased focus on establishing an evidence base of effective psychosocial treatments in ADHD (Pelham 2008). In 1998, Pelham and colleagues reviewed relevant literature and concluded that "Behavioural Parent Training barely met criteria for well-established treatment" but met criteria for "a probably efficacious treatment" (Pelham 1998). A decade later, Pelham et al updated this review suggesting that their findings extend the earlier review and demonstrate that behavioural interventions (including behavioural parent training, behavioural classroom management and intensive summer programme-based peer interventions) "are supported as evidence-based treatments for ADHD” (Pelham 2008).

Children diagnosed with ADHD or HKD often have multiple problems, including comorbid disorders such as anxiety, depression and oppositional defiant disorder, as well as relationship difficulties, so multimodal treatment appears to be appropriate (Wells 2000; Taylor 2004; NICE 2008). This might be why, for children with less severe symptoms, safety and user preference may lead to 
behavioural interventions may being used as a first choice, despite the small advantage of medication over psychological intereventions (Santosh 2005). There are many reasons for considering psychosocial interventions in ADHD, including uncertainity about the long-term effectiveness of stimulants; minimal clinical benefits of medication; nonresponsiveness to medication; weak responsiveness to medication; intolerance to medication; clinical needs of younger children, and ethical and other objections to the use of medication (NICE 2008).

\section{Description of the intervention}

Parent training programmes are psychosocial interventions aimed at training parents in behavioural or cognitive behavioural techniques they can use to manage their children's challenging behaviour. The programmes vary in their style and content but are generally manual-based and may involve discussion and the use of video and role play. One example is Webster-Stratton's Incredible Years programme (Webster-Stratton 1998). In addition to the behavioural or cognitive behavioural content fundamental to generic parent training programmes, ADHD-focused parent training often includes psychoeducational components about ADHD and how its presence affects a child's functioning and behaviour (Pliszka 2007). The programmes are typically delivered to groups of parents and usually comprise 10 to 20 weekly sessions of one to two hours covering a range of areas that include the nature of $\mathrm{ADHD}$, positive reinforcement skills (for example, attending carefully to appropriate behaviour and play, as well as how to ignore unwanted behaviour), reward systems, the use of 'time out', liaison with teachers and planning ahead to anticipate problems (Pliszka 2007).

\section{How the intervention might work}

The main aim of parent training, for children with conduct problems, is “to reduce children's problem behaviour by strengthening parent management skills" (Hartman 2003). Parent training interventions are mainly based on behaviour management principles that arise from social-learning theory. They are based on the theory that behaviour can be influenced by its antecedents and consequences and that parents can learn how to intervene with both of these. Parent training usually comprises structured programmes that are delivered in a standardised way by professionals trained in behaviour management theory and practice (Kazdin 1997; NICE 2006a).

More specifically, in families with children who have ADHD parent training may be aimed at improving the parents' understanding of ADHD or increasing their behaviour management skills, or both. Parents may also learn self-management skills aimed at reducing stress and increasing resilience.
In people with ADHD, it is hypothesised that deficits in the brain's executive functioning might result in excessive impulsivity and an altered motivational state may cause delay aversion (Solanto 2001; Sonuga-Barke 2003b). Hypothetically, to improve ADHD symptoms parent training could be designed to work on both of these areas, through cognitive work on self-regulation and through motivational interventions focusing on improving delay tolerance (Sonuga-Barke 2003b).

\section{Why it is important to do this review}

Longitudinal research suggests that hyperactivity, in particular, is a risk factor for future problems (Taylor 1996; Sourander 2005). Unfavourable long-term outcomes include educational and occupational impairment (Weiss 1985; Mannuzza 1997), an increased risk of antisocial personality disorder and substance misuse (Mannuzza 1998; Rasmussen 2000) and an increased risk of "psychiatric diagnosis, persisting hyperactivity, violence and other conduct problems and social and peer problems" (Taylor 1996). Problem behaviours are viewed in two broad dimensions. Externalising problems include conflict with others, aggression, rulebreaking and oppositional behaviour, whereas internalising problems are those where stress is directed against the self, such as anxiety, depression, somatic problems and social withdrawal (Sourander 2005). The National Survey of Health and Development, a $40-$ year cohort study, followed up 3652 adolescents with externalising problems. It found that those with externalising behaviour problems are impaired in their health and social development in multiple ways, with considerable impact on themselves, their families and society throughout adult life (Coleman 2009).

Parent training can improve behaviour in children with conduct disorder (Kazdin 1997; NICE 2006a) and children with behaviour problems (Barlow 1997). There is also some support for the effectiveness of group-based parenting programmes in improving the emotional and behavioural adjustment in children under the age of three years (Barlow 2010). Furthermore, it may be effective in children who have both conduct disorder and the ADHD core symptoms of inattention, hyperactivity and impulsivity (Hartman 2003).

The economic cost of antisocial behaviour to society is considerable and cuts across multiple agencies. When costs were applied to data from the Inner London longitudinal study, it was reported that those with conduct disorder cost 10 times more than those without (Scott 2001b). Parent training programmes for conduct disorder are effective (Kazdin 1997; Scott 2001a; NICE 2006a; Hutchings 2007; Scott 2007) yet until relatively recently they attracted little funding. This was despite the fact that the effect size of these interventions is comparable to that of antidepressant medication in depressed adults (Scott 2007).

Although comorbidity exists between ADHD and conduct disorder or oppositional defiant disorder, it is not clear that parent training in children with ADHD, with or without comor- 
bid conduct disorder or oppositional defiant disorder, is effective for reducing antisocial behaviour or ADHD symptoms. Although NICE recommend parent training as a treatment intervention in ADHD (NICE 2008), this recommendation is based on studies of children under 12 years with conduct disorder rather than with ADHD (NICE 2006a). They nonetheless recommend that clinical services provide all parents or carers of preschool children a parent training or education programme as first-line treatment. They also suggest that parents or carers of school age children with moderate ADHD symptom impairment be offered parent training programmes and that school-aged children be offered a group treatment programme involving cognitive behavioural therapy or social skills training, or both (NICE 2008).

Given the high comorbidity between ADHD and conduct disorder or oppositional defiant disorder, it is understandable that NICE have made these recommendations but the relationship between parent training and ADHD needs to be examined in its own right. The mechanism for development of behaviour problems might be different for the two conditions.

\section{O B J E C T I VES}

To determine whether parent training interventions are effective in reducing ADHD symptoms and associated problems (for example, disruptive behaviour disorders or specific impairments such as learning difficulties) in children and young people aged five to 18 years with ADHD.

\section{METHODS}

\section{Criteria for considering studies for this review}

\section{Types of studies}

Randomised controlled trials (RCTs), including quasi-randomised trials where sequence generation was, for example, by birth date or alternate allocation, that contain at least one measure of ADHDrelated behaviour. As specified in the protocol, we did not include trials which did not report any outcome data on outcomes relating directly to the child's own behaviour or well being (ADHD-related or not).

\section{Types of participants}

Children and young people aged five to 18 years (or with a mean age above five years) in whom the main problem was ADHD (or hyperkinetic disorder) diagnosed using DSM or ICD operationalised diagnostic criteria. The diagnoses must have been clinical diagnoses by specialists with or without the use of semi-structured or structured interview instruments. Acceptable diagnoses included:

- Attention-Deficit/Hyperactivity Disorder (DSM III-R, DSM-IV) (APA 1987; APA 1994);

- Attention Deficit Disorder (DSM III) (APA 1980);

- Hyperkinetic Disorder (ICD-9, ICD-10) (WHO 1977; WHO 1992).

Parents of these children were the recipients of the parent training intervention as defined below.

\section{Types of interventions}

Parent training programmes where the intervention was designed to train parents in behavioural or cognitive behavioural, or both, interventions to improve the management of their child's ADHDrelated difficulties. The term 'parent training' includes:

- group-based interventions;

- interventions for individual parents, or for a couple;

- the combination of individual or couple and group interventions; and

- parents acting as the main mediators of the intervention with an additional component involving teacher(s) trained in behavioural management.

We did not include trials which did not report any outcome data on outcomes relating directly to the child's own behaviour or well being (ADHD-related or not). We also excluded trials where direct interventions with the children were used. This was to separate out the effect of parent training and the effect of the direct behavioural intervention with the child and eliminate the possibility of interaction between them.

However, we included trials in which drug treatments were used alongside parent training interventions (that is parent training plus medication versus medication alone) and we planned subgroup analysis of trials in which drug treatments were used.

We recorded details on all interventions permitted to the control group or indeed the intervention groups (that is existing drug or therapy regimes).

\section{Types of outcome measures}

\section{Primary outcomes}

- * Change in the child's ADHD symptom-related behaviour in home setting; for example, Conner's or SNAP questionnaires (Swanson 1983; Conners 1998a)

- * Change in the child's ADHD symptom-related behaviour in school setting; for example, Conner's Teacher Rating Scale (Conners 1998b) 
- * Changes in the child's general behaviour; for example, Achenbach Child Behaviour Checklist (Achenbach 1991)

All primary outcomes related to participant children, not to parent outcomes (for example, reduction of parental stress), so studies with only parent outcomes were excluded. See also Potential biases in the review process.

\section{Secondary outcomes}

- * Academic achievement of children as measured through school test results or general tests of language or development

- *Adverse events (these could include emotional or psychological trauma of any kind, such as might be suffered by a parent with a history of physical abuse experiencing flashbacks in a discussion about physical chastisement, or parents for whom parent training causes an increase in anxiety or depression about their own skills)

- *Changes in parenting skills; for example, The Parenting Clinical Observation Schedule (Hill 2008)

- *Parental stress; for example, the Parenting Stress Index (Abidin 1995)

- Parental understanding of ADHD; for example, ADHD

Knowledge \& Opinion Scale (Rostain 1993)

Outcome measures may be reports by a clinician, parent, teacher or trained investigator. Instruments used must be published in a peer-reviewed journal following validation in the population, that is tested for validity in children or young people with ADHD and found to measure the change that they set out to measure.

Outcomes marked by asterisks indicate outcomes planned for inclusion within a 'Summary of findings' table (Schünemann 2008).

\section{Search methods for identification of studies}

\section{Electronic searches}

We searched the following electronic databases.

- Cochrane Central Register of Controlled Trials

(CENTRAL) (2010 Issue 3) (searched 14 September 2010).

- MEDLINE: 1950 to current (searched 14 September 2010).

- EMBASE: 1980 to 2010 Week 36 (searched 24 September 2010).

- CINAHL: 1937 to current (searched 13 September 2010).

- PsycINFO: 1806 to September week 12010 (searched 14

September 2010).

- Dissertation Abstracts International, searched through

Dissertation Express (14 September 2010).

- metaRegister of Controlled Trials (searched 14 September 2010).
All search strategies used for this review appear in Appendix 1, Appendix 2 and Appendix 3. Searches were run between 2002 and September 2010, during which time there were a number of changes to databases and platforms or database providers. For the most recent searches, the RCT filter in MEDLINE was updated to reflect the revised filter published in the Cochrane Handbook for Systematic Reviews of Interventions (Lefebvre 2008).

No language filters were applied.

\section{Searching other resources}

We checked references in previous reviews and the bibliographies of included and excluded studies. We contacted authors and known experts to identify any additional or unpublished data.

\section{Data collection and analysis}

\section{Selection of studies}

Two researchers working in pairs undertook initial screening of abstracts and titles from the search independently to identify potential trials for inclusion. The first author (MZ) screened abstracts and titles at each of several searches conducted between 2002 and 2010, in tandem with either JD, HJ or AY. Studies were then independently assessed and selected for inclusion by the same pairs of review authors. We reached consensus by discussion. We made a flow chart of the process of trial selection in accordance with the PRISMA statement (Moher 2009).

\section{Data extraction and management}

\section{Data extraction}

Two of four authors (MZ, CT, HJ and JD) extracted data independently using data extraction sheets that had been previously piloted to check for reliability in extracting the relevant data. We stored and organised citations in ProCite bibliographic software.

\section{Data collection}

When more than two treatment arms were included in the same trial, we described all arms.

We collected the following data for all trial arms.

1. Descriptive data, including participant demographics (age, gender, baseline measures of school achievement, social and economic status).

2. Intervention characteristics (including delivery, duration and within-intervention variability).

3. Other interventions received (including delivery and duration).

Parent training interventions for Attention Deficit Hyperactivity Disorder (ADHD) in children aged 5 to 18 years (Review)

Copyright (C) 20I I The Cochrane Collaboration. Published by John Wiley \& Sons, Ltd. 
4. Outcome measures listed above.

\section{Assessment of risk of bias in included studies}

At least two review authors independently assessed the risk of bias in each included study using the Cochrane Collaboration's risk of bias tool (Higgins 2008a; Higgins 2008b). Each study was assessed in relation to the six domains listed below.

Ratings given were: low risk of bias, high risk of bias or unclear risk of bias.

\section{Sequence generation}

Description: the method used to generate the allocation sequence is described in sufficient detail so as to assess whether it should have produced comparable groups. The review authors' judgment: was the allocation sequence adequately generated?

\section{Allocation concealment}

Description: the method used to conceal allocation sequence is described in sufficient detail to assess whether intervention schedules could have been foreseen in advance of, or during, recruitment. The review authors' judgment: was allocation adequately concealed?

\section{Blinding}

Description: any measures used to blind participants, personnel and outcome assessors are described so as to assess knowledge of any group as to which intervention a given participant might have received. The review authors' judgment: was knowledge of the allocated intervention adequately prevented during the study?

\section{Incomplete outcome data}

Description: when studies did not report intention-to-treat analyses, we made attempts to obtain missing data by contacting primary investigators. We extracted and reported data on attrition and exclusions as well the numbers involved (compared with total randomised), reasons for attrition/exclusion where reported or obtained from investigators, and any re-inclusions in analyses performed by review authors. Review authors' judgment: were incomplete data dealt with adequately by the review authors? (See also Dealing with missing data.)

\section{Selective outcome reporting}

Description: attempts were made to assess the possibility of selective outcome reporting by investigators, including searching for trial protocols via registers. The review authors' judgment was based on the question: are reports of the study free of suggestion of selective outcome reporting?

\section{Validity and reliability of outcome measures used}

Description: were the outcome measures standardised and validated for the population?

\section{Other sources of bias}

Description: was the study apparently free of other problems that could put it at a high risk of bias?

During data extraction, we identified potential 'contamination' effects between participants in different arms of a study as an additional risk of bias (see Characteristics of included studies below).

\section{Measures of treatment effect}

Only limited meta-analysis was possible in this review. The methods and choices we made regarding measures of treatment effect and other issues pertinent to meta-analysis can be viewed in Appendix 4 for binary and categorical data as no such data were identified in studies included within this review.

\section{Continuous data}

When continuous outcomes were measured using the same scale across studies, we calculated an overall mean difference (MD) and 95\% confidence interval (CI). When the same continuous outcome was measured differently across studies, we calculated an overall standardised mean difference (SMD) and 95\% CI. We calculated SMDs using Hedges $g$.

\section{Unit of analysis issues}

See Appendix 4.

\section{Dealing with missing data}

Where necessary, we contacted the corresponding author of a study to supply any unreported data. We planned to contact authors to obtain outcomes for any studies in which only per protocol analysis had been undertaken but, in the event, some authors undertook intention-to-treat analysis (for example, van den Hoofdakker 2007) and for others the number of dropouts was low, so overall this protocol decision was felt to be unnecessary for the current version of this review.

There was only one trial for which we sought additional outcome data (Fallone 1998). This was because numerical data for nonsignificant outcomes or numerical data for the whole sample (as compared with the subset of participants who attended more than $50 \%$ of parent training sessions) were absent from the text of the trial report. Our repeated attempts to make contact with this author between 2007 and 2011 were unsuccessful. 


\section{Assessment of heterogeneity}

We assessed the extent of heterogeneity using the three methods suggested by the Cochrane Handbook for Systematic Reviews of Interventions (Deeks 2008): visual inspection of forest plots, the Chi ${ }^{2}$ statistic (increasing the level of significance to 0.10 to avoid underestimating heterogeneity) and using the $\mathrm{I}^{2}$ statistic, which describes the "percentage of the variability in effect estimates that is due to heterogeneity rather than sampling error (chance)" (Higgins 2002; Higgins 2003). It is advised that the thresholds of the $\mathrm{I}^{2}$ statistic might be misleading and the following guide is offered:

- $0 \%$ to $40 \%$ might not be important;

- $30 \%$ to $60 \%$ may represent moderate heterogeneity;

- $50 \%$ to $90 \%$ may represent substantial heterogeneity;

- $75 \%$ to $100 \%$ represents considerable heterogeneity.

We bore in mind that the "importance of the observed value of $\mathrm{I}^{2}$ depends on (i) magnitude and direction of effects and (ii) strength of evidence for heterogeneity (for example, $\mathrm{P}$ value from the Chi 2 test, or a confidence interval for $\mathrm{I}^{2}$ )" (Higgins 2008a). Clinical heterogeneity is discussed in the Results, Overall completeness and applicability of evidence and Authors' conclusions.

\section{Assessment of reporting biases}

As studies were few and opportunities for meta-analysis fewer within this review, our plans for funnel plots and related methods were not feasible but we have archived them for future updates (Appendix 4).

As planned in our protocol, we attempted to address the issue of selective outcome reporting by seeking out the protocols of the included studies (within trial registries, conference proceedings etc) and by internal evidence within the published studies.

\section{Data synthesis}

\section{Outcome data}

Where sufficient clinical and methodological homogeneity existed between trials, we pooled results. We remained mindful of the dangers of interpreting findings of single or even multiple studies in the absence of meta-analysis. Where meta-analysis was not possible, for example where outcomes measured different domains such as 'ADHD core symptoms' and 'educational achievement', we provided the reasons and reported investigators' findings narratively.

Review Manager 5.0 (RevMan 2008) was used to conduct metaanalysis where feasible. We calculated overall effects using inverse variance methods (we planned to undertake both fixed-effect and random-effects model meta-analyses and report data accordingly). We anticipated that included studies would yield heterogeneous data because of diagnostic variability in ADHD and differences in the parent training models used by different researchers, and we were correct in our assumptions.

\section{Types of analyses}

See Appendix 4.

\section{Multiple arms}

All relevant outcomes for all eligible trial arms were reported in the review.

When two or more eligible intervention groups were compared to an eligible control, the review authors considered (as planned in the protocol) combining data for interventions provided each met all inclusion criteria and did not involve unacceptable adjunct treatments, for example direct work with children. We used the methods recommended in the Cochrane Handbook for Systematic Reviews of Interventions (Higgins 2008c) to combine data from the two 'active treatment' arms of the three-arm trial conducted by Fallone 1998 (parent training versus parent training and selfmanagement versus control).

At protocol stage we planned the following: "If a single eligible intervention group is compared to multiple eligible control groups, 'no-treatment' controls will be chosen over other groups for comparison and inclusion in meta-analyses (Lipsey 2001). For studies that do not have no-treatment condition, the most appropriate eligible alternative will be chosen (see list of comparisons, above)". This decision led us to accept the study by Lehner-Dua 2001 as we judged her parent support group to be synonymous with an 'attentional control'. We could not be certain of trials such as Lauth 2007, wherein the 'parent support' arm of the trial involved intensive discussion of themes pre-chosen by intervention organisers to mirror those being taught in the more active parent training intervention arm.

\section{Multiple measures}

When a single study provided multiple measures of the same outcome (for example, two measures for ADHD symptoms), we planned at protocol stage to average the effects from the outcomes to arrive at a single effect for use in the meta-analysis. In the event, this was not feasible in the one instance it occurred. We therefore reported separate subscales of the ADDES-HOME scale (Hyperactivity and Inattentiveness) in the one trial using these measures (Fallone 1998). These scales measure different aspects of ADHD symptomatology and it was therefore inappropriate to combine them in a meta-analysis.

\section{Subgroup analysis and investigation of heterogeneity}

We did not identify enough trials to conduct any subgroup analyses. Plans for assessment of statistical heterogeneity remain in Appendix 4. Clinical heterogeneity is discussed in Results; 
Overall completeness and applicability of evidence and Authors' conclusions.

\section{Sensitivity analysis}

See Appendix 4.

\section{Qualitative data}

We planned to report qualitative data from the included studies to better understand the delivery of interventions, uptake by participants and context; however we did not identify any such data reported in relation to studies included within this review.

\section{R E S U L T S}

\section{Description of studies}

\section{Results of the search}

We ran searches six times in the period during which the review was developed: four times after publication of the original 2001 protocol (by Jo Abbott in July 2002; Eileen Brunt in June 2004; Jo Abbott in November 2006; and Lynn Turner in May 2008) and twice after the publication of the revised 2009 protocol (by Jo Abbott in May 2009; Jo Abbott and Margaret Anderson in September 2010).

Results were vetted by different review author pairs at different times. These were: MZ and JD for searches up to 2006; MZ and $\mathrm{HJ}$ for searches in May 2008; MZ, HJ and AY for searches in 2009; and MZ and JD for searches in 2010. All decisions on eligibility were made by consensus within the author team.

Total numbers for searches are as follows.

- Inception to $2004-6671$.

- 2004 to $2006-1542$.

- 2006 to 2008 - 1579 .

- 2008 to $2009-1280$.

- 2009 to 2010 - 1889.

Minus duplicates that were automatically rejected by Procite software (but not counting overlaps between searches or duplicates later excluded manually), we identified 12,691 records through search strategies (see Appendix 1, Appendix 2 and Appendix 3). After screening of titles and abstracts, full texts of 112 papers were obtained over time. Five unique studies cited in six papers met our inclusion criteria. Many investigators were contacted to supply further data before decisions on inclusion could be made. We excluded 74 unique studies (reported in 89 documents). See the table of Excluded studies for further details.

\section{Included studies}

Five studies published between 1993 and 2010 met the inclusion criteria (Blakemore 1993; Fallone 1998; Lehner-Dua 2001; van den Hoofdakker 2007; Mikami 2010).

\section{Location}

Four of the five studies were conducted in North America. One study was conducted in Canada at The Learning Centre, Calgary, Canada (Blakemore 1993). Three studies were conducted in the USA: one in Memphis, Tennessee (Fallone 1998); one at the Hofstra University's Centre for Psychological Evaluation, New York (Lehner-Dua 2001); and one at the University of Virginia in Charlottesville, Virginia (Mikami 2010). The fifth study was conducted in the Netherlands at an outpatient clinic in Groningen (van den Hoofdakker 2007).

\section{Design}

All included studies were described by the investigators as randomised controlled trials. Four employed a stratified 'block' design (Blakemore 1993; Fallone 1998; van den Hoofdakker 2007; Mikami 2010) and the fifth (Lehner-Dua 2001) randomised by individual participants.

Two studies involved three arms (Blakemore 1993; Fallone 1998); the remaining three were, for the purposes of this review, twoarmed intervention studies. Mikami 2010 did involve a third group but as it was a normative comparison group of children without a diagnosis of ADHD the study was treated within this review as a parallel group study.

\section{Sample size}

Overall, sample sizes were small, ranging from 24 participants ( Blakemore 1993) to 96 (van den Hoofdakker 2007); the remaining studies comprised 54 (Fallone 1998), 48 (Lehner-Dua 2001) and 62 (Mikami 2010) participants respectively.

\section{Participants}

To be included in this review, ADHD had to be the main focus of the trial and participants had to have a clinical diagnosis of ADHD made by a specialist using operationalised diagnostic criteria of the DSM-IV, or its earlier versions, or a diagnosis of hyperkinetic disorder using ICD-10 (see under 'Inclusion criteria' below).

Participants included within the review ranged in age from four to 13 years old. Ranges, means and standard deviations, where provided, were as follows.

Blakemore 1993, six to 11 years (no other information supplied); Fallone 1998, five to 9 years (means: group $1=6.94(\mathrm{SD}=1)$; group $2=6.56(\mathrm{SD}=1.03)$; group $3=6.88(\mathrm{SD}=1.36))$; Lehner-Dua 2001 , six to 10 years $($ median $=8.0)$; Mikami 2010 , six to 12 years 
(mean $=8.9)$; van den Hoofdakker 2007, four to 12 years (mean $=7.4, \mathrm{SD}=1.9$ ).

All investigators with the exception of Blakemore 1993 supplied data on gender. They each reported a majority of male children (179 boys versus 65 girls across the four studies in which this demographic was reported).

Most children entered the studies on medication for ADHD symptoms (see below for further details, as reported in the individual studies). In general, where reported, participants had to be stablised on medication throughout the trial and this was established prior to randomisation. We also have to assume that medication, where used, was used in the same way across the intervention and control groups.

\section{Inclusion criteria}

Blakemore 1993 used DSM-III-R criteria for ADHD. The children had to demonstrate evidence of ADHD in a wide range of situations and the problems must have been evident before the age of six years. Fallone 1998 included participants who had to be diagnosed with ADHD using DSM-IV criteria. Fallone also required a high level of maternal stress for inclusion in the training programme. Lehner-Dua 2001 and van den Hoofdakker 2007 used DSM-IV criteria. Mikami 2010 reported that DSM-IV criteria were used (that is the proportion of those of Combined type (ADHD-C) and Inattentive type (ADHD-I) were reported). In this study, diagnoses were further reinforced and refined using the Child Symptom Inventory (CSI) (Gadow 1994) and confirmed by parental interview using the K-SADS-PL (Kaufman 1997). van den Hoofdakker 2007 included participants who met DSMIV criteria for ADHD, had an IQ > 80 (full scale IQ of the WISCIII-R; for children under the age of six years the Full Scale IQ of the QWPPSI-R) and were four to 12 years old. In addition, both parents (if present) had to be willing to participate in the behavioural parent training program. All participants in the trial were offered a comprehensive package of routine clinical care (RCC) including psychological treatment, pharmacological treatment and crisis management, where necessary, with the parent training component being offered as the only difference between the groups. The control group were also offered the intervention after a waitlist period, described as shorter than the one they might have faced had they not participated in the trial (it was hoped that this would improve retention in the control group).

\section{Exclusion criteria}

Blakemore 1993 excluded participants if there was evidence of a serious neurological difficulty in the child, evidence of a serious marital difficulty, or where the child met criteria for conduct disorder. Fallone 1998 excluded participants with "mental retardation" or pervasive developmental disorder. Lehner-Dua 2001 did not specify any exclusion criteria. van den Hoofdakker 2007 did not specify any exclusion criteria, wishing for a 'naturalistic' intake, and its authors made clear that they thus anticipated (and found) a higher rate of comorbidities than other similar studies of children with ADHD. Mikami 2010 excluded children with pervasive developmental disorders, full scale IQ $<70$ or verbal IQ $<75$. No child could be receiving other psychosocial treatment for social and behavioural issues at the time of the study; however, educational interventions were allowed.

\section{Intervention and control conditions}

Blakemore 1993 allocated participants to two active experimental arms, one for group parent training and one for individual parent training, with a waitlist control group who were offered the group intervention at the end of the study. Fallone 1998 included two experimental arms, one for behavioural parent training alone, the other for behavioural parent training combined with self management, again with a waitlist control group offered intervention at the end of the study. Mikami 2010 included one intervention group that received 'Parental friendship coaching' (PFC), a programme that resembled other parent training programmes for the first two sessions then focused on developing social skills in children, versus a no treatment control group which at the close of the study received a summary session on the programme content of the intervention (PFC).

The duration of the parent training varied: 12 weekly, two hour sessions (Blakemore 1993, group treatment); 12 weekly, one hour sessions (Blakemore 1993, individual treatment); 12 two hour sessions spread over five months (van den Hoofdakker 2007); nine weekly, two hour sessions (Lehner-Dua 2001); eight weekly, 50 minute sessions (Fallone 1998, parent training treatment); and eight weekly one and a half hour sessions (Fallone 1998, parent training and self management treatment). Mikami 2010's intervention, PFC, was delivered in eight group sessions of 90 minutes each.

Lehner-Dua 2001 stood out in this review by offering a parent support group as a placebo control, whilst van den Hoofdakker 2007 explicitly offered 'treatment as usual' as control. We judged that, as in the other studies, all participants remained on existing pharmacological or other treatment regimes and no child appeared to have been denied such treatment; controls were quite similar in this respect.

\section{Follow-up}

Blakemore 1993 had two follow-up assessments at three and six months. No other study reported collecting data at later time points, or planning to do so, and this may be explained by waitlist conditions or financial constraints, or both.

\section{Outcomes}


Instruments used to measure primary and secondary outcomes within the included studies varied widely.

\section{Change in the child's ADHD symptom-related behaviour in home setting}

The Conners' Parent Rating Scale (Conners 1970) was used by Blakemore 1993. Similarly, the ADHD Index subscale of the Conners' Parent Rating Scale-Revised Short Form (CPRS-R-S) (Conners 2001) was used by van den Hoofdakker 2007. Fallone 1998 employed the Hyperactive-Impulsive and the Inattentive scales of the ADDES-Home (McCarney 1995) for this outcome. Lehner-Dua 2001 used the Behaviour Assessment System for Children (BASC) (Reynolds 1998) to measure parents' perceptions of child behaviour.

\section{Change in the child's ADHD symptom-related behaviour in school setting}

Fallone 1998 used the ADDES-School for this outcome ( McCarney 1995), which involves both a Hyperactive-Impulsive and an Inattentive Scale.

\section{Changes in the child's general behaviour}

Externalising and Internalising subscales of the Dutch version of the Child Behaviour Checklist (CBCL) (Achenbach 1991) were used by van den Hoofdakker 2007 and the Achenbach Child Behaviour Checklist (Achenbach 1991) was also used by Blakemore 1993 and Fallone 1998 (who also used a total CBCL score). Investigators involved in Blakemore 1993 also used the Eyberg Child Behaviour Inventory (ECBI) (Eyberg 1999).

Mikami 2010 used the teachers' version of the Social Skills Rating System (SSRS) (Gresham 1990) and the Dishion Social Acceptance Scale (DSAS) (Dishion 2003). The latter measures the extent to which observers feel a child is liked and accepted or disliked and rejected by peers.

Mikami 2010 used the parents' version of the Social Skills Rating System (SSRS) (Gresham 1990).

\section{Academic achievement measured through school test results}

Academic achievement was not measured in any study included within this review.

\section{Adverse events}

No study reported adverse events in any way.

\section{Changes in parenting skills}

Lehner-Dua 2001 used the Parenting Sense of Competence (PSOC) (Johnston 1989) for this outcome.

\section{Parental stress}

Blakemore 1993, Lehner-Dua 2001 and van den Hoofdakker 2007 all used versions of the Parenting Stress Index (PSI) (Abidin 1986) or short form (PSI-SF) (Abidin 1990) for this outcome; Fallone 1998 measured parental stress using the Revised Symptom Checklist (SCL-90-R) (Derogatis 1994).

\section{Parental understanding of ADHD}

No study included within this review measured this outcome specifically.

\section{Measures in included studies that were not used in this review}

A number of outcomes reported in the studies included in this review are not reported here, either because they did not fit the protocol for the review or they were from unpublished instruments, generally devised by the investigators of the studies themselves (for example, a structured interview with parents (Blakemore 1993)). In a US study primarily focused on assisting parents to help their children form social bonds (Mikami 2010), several outcome measures were unusable in our review including: the 'Quality of Play Questionnaire (QPQ) and Playdates Hosted', both cited in an unpublished manuscript (Frankel 2003); the 'Child Friendships at Follow-up' questionnaire (global five point questionnaire completed by parent, devised by investigators); the Parental Behaviour in Playgroup (socialising, facilitation and corrective feedback, videotapes coded by blinded observers on a scale of 10); the Parental Behaviour in Parent-child Interaction (coded as above using a Likert scale from zero to three).

\section{Treatment fidelity}

Parent training for ADHD is typically delivered following training of staff via a manualised treatment protocol. Amongst studies included within this review, only Fallone 1998 described efforts to ensure treatment fidelity across all sessions.

\section{Excluded studies}

See Characteristics of excluded studies for details of 74 excluded studies, data for which we found in 89 publications.

Many studies which appeared (by title or even abstract) to be of interest did not ultimately meet our inclusion criteria. Some studies were excluded for more than one reason. Primary reasons for exclusion are listed below. 


\section{Lack of random allocation or a control group}

Randomised and quasi-randomised studies were eligible for this review. Of the 68 intervention studies identified and inspected for this review, 64 were (at a minimum) controlled intervention studies.

Seven studies were excluded because participants were not randomly or quasi-randomly allocated to intervention groups ( Anastopoulos 1993; Bandsma 1997; Taylor 1998; Weinberg 1999; Ercan 2005; Salbach 2005; Gibbs 2008). In these studies, it was usually found (sometimes only after contact with investigators) that allocation was by self-selection on the part of parents, or that groups had been formed by clinical severity alone (Bandsma 1997). Four studies were excluded because, although they were intervention studies, they proved to have no control group at all (Pollard 1983; Danforth 1998; Arnold 2007; Hautmann 2009).

Fourteen studies included no eligible control group (Barkley 1992; Sanders 2000b; Barkley 2001; Montiel 2002; Corrin 2003; Hall 2003; Isler 2003; Corkum 2005; McGoey 2005; Fabiano 2006; Grimm 2006; Markie-Dadds 2006; Lauth 2007; Cummings 2008). The latter group of studies generally compared active interventions only; in some cases they also failed to meet diagnostic criteria for children.

\section{Direct intervention of therapist with child}

Fourteen studies (Horn 1991; MTA 1999; Abikoff 2004; Chronis 2004; Springer 2004; Waschbusch 2005; Miranda 2006; Bogle 2007; Chacko 2007; Dubbs 2008; Guo 2008; Molina 2008; van der Oord 2008; Coughlin 2009) were excluded because their intervention directly involved the child.

\section{Problems with diagnosis or diagnostic criteria}

Sixteen studies were excluded because, although investigators may have described the children as having ADHD, there was no clear ADHD diagnosis made that met our inclusion criteria, or the children were too young for this review (O'Leary 1976; Dubey 1978; Pisterman 1989; Pisterman 1992a; Beyer 1994; Connell 1997; Barkley 2000; Sanders 2000a; Sonuga-Barke 2001; Barkley 2002b; Bor 2002; Sonuga-Barke 2004; Gustis 2007; Heriot 2008; Jones 2008; Larsson 2008). Readers interested in the results of studies that focus primarily on the young with subclinical behavioural problems may be interested in the results of another Cochrane review, when complete (Furlong 2010).

Six studies were excluded as the children had behavioural problems due to other conditions including oppositional defiant disorder (ODD) or conduct disorder (CD) (Nixon 2001; Nixon 2003; Lavigne 2008; Scott 2010), pervasive developmental disorders ( Aman 2010) or being 'gifted' (Morawska 2009).

\section{Focus of the study}

Two studies were excluded due to intervention type: Chronis 2006 because the intervention targeted maternal depression amongst mothers of children with ADHD, and was assessed as not meeting the inclusion criteria for parent training; and Wolraich 2005 because the intervention sought to improve communication between parents, teachers and clinicians rather than to manage child behaviour.

Two studies narrowly missed inclusion (Scott 2001a; Scahill 2006) because ADHD was not the main focus of the trial, which rendered data interpretation problematic. Scahill 2006 recruited children with tic disorders and disruptive behaviour from a specialised tic disorders clinic, and specifically excluded children with untreated ADHD. This yielded a subset of children with comorbid, medicated ADHD (investigators further responded to our request for subset data with the view that this sample was too small to analyse meaningfully (Scahill 2008)). In contrast, Scott 2001a took pains to exclude children diagnosed with the ICD-10 diagnosis of 'hyperkinetic syndrome' precisely because he wished to exclude medicated children with ADHD from his study. We have recently learned from a personal communication that, despite the fact that the published paper does not refer to any child having a diagnosis of ADHD, a substantial proportion of participants did, posthoc, merit this diagnosis (Scott 2011) and that unpublished data for this subset exist. Yet these participants, given the exclusion criteria, were solely unmedicated children with ADHD. Thus we concluded that, in the absence of an underpinning focus on ADHD, a risk of bias (in that children with ADHD will have been excluded from both studies who would have been included in other included studies) might 'skew' the results of this review.

\section{No child outcomes}

Three studies (Odom 1996; Corkum 1999; Treacy 2005) were excluded because although the studies involved parents of children with $\mathrm{ADHD}$, and sequence generation was adequate for the eligible intervention and control groups, no outcomes relating to the child's behaviour or well being (ADHD-related or not) were reported. In these studies, outcomes typically included parental stress, parental knowledge of ADHD and willingness to medicate children or seek counselling.

\section{Opinion pieces and review articles}

Six studies (Ellis 2009; Fagan Rogers 2009; Hauth-Charlier 2009; Reeves 2009; Schoppe-Sullivan 2009; Baker-Ericzen 2010) were excluded because they were found to be review articles, opinion pieces or other types of studies when obtained and inspected. For example, Schoppe-Sullivan 2009, whilst tagged as a randomised controlled trial in MEDLINE, proved to be an observational study considering parents' own ADHD symptoms in relation to their parenting practices. 


\section{Risk of bias in included studies}

See also Figure 1 and Figure 2.

Figure I. Methodological quality summary: review authors' judgements about each methodological quality item for each included study.

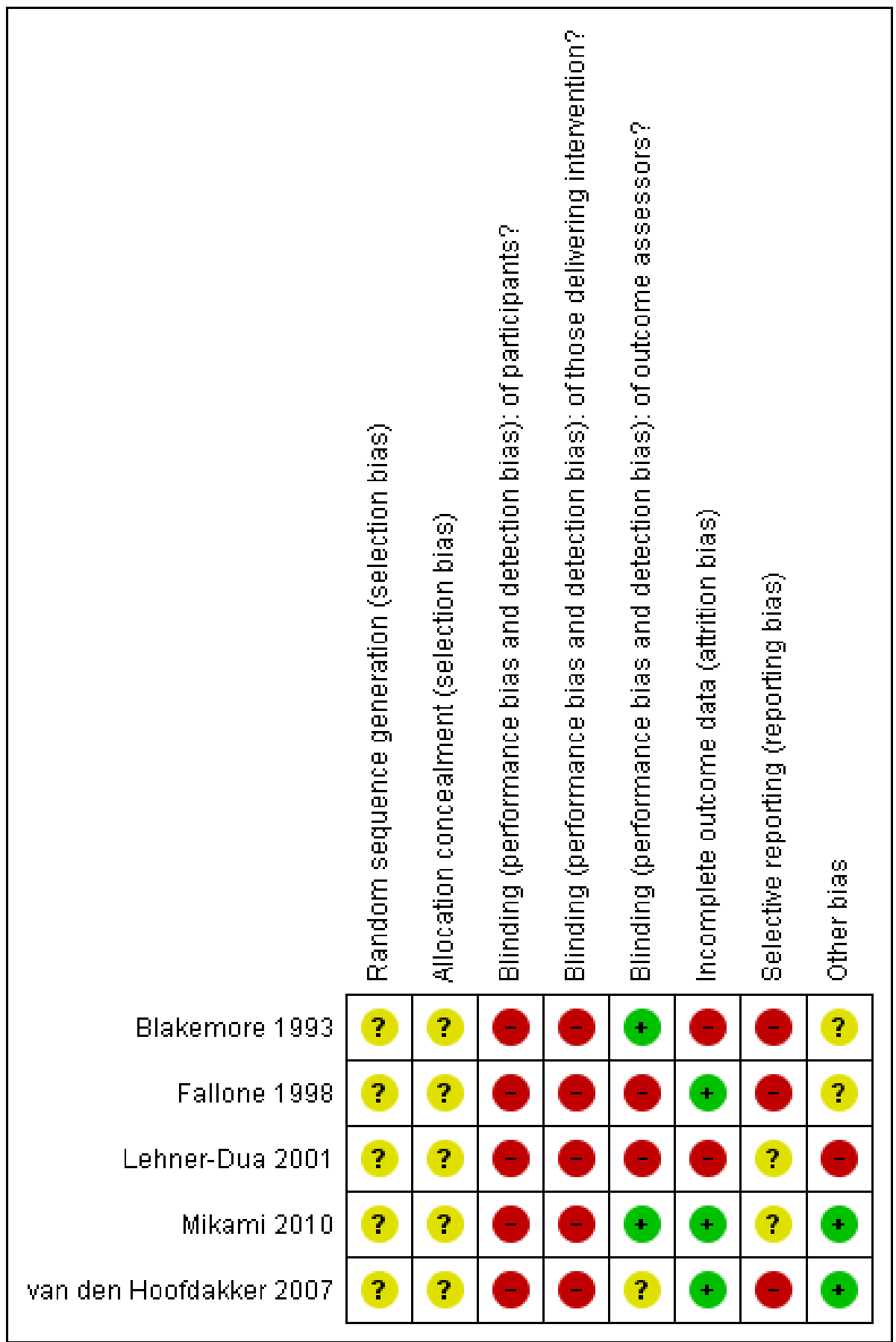

Parent training interventions for Attention Deficit Hyperactivity Disorder (ADHD) in children aged 5 to 18 years (Review) Copyright (C) 20I I The Cochrane Collaboration. Published by John Wiley \& Sons, Ltd. 
Figure 2. Risk of bias graph: review authors' judgements about each risk of bias item presented as percentages across all included studies.

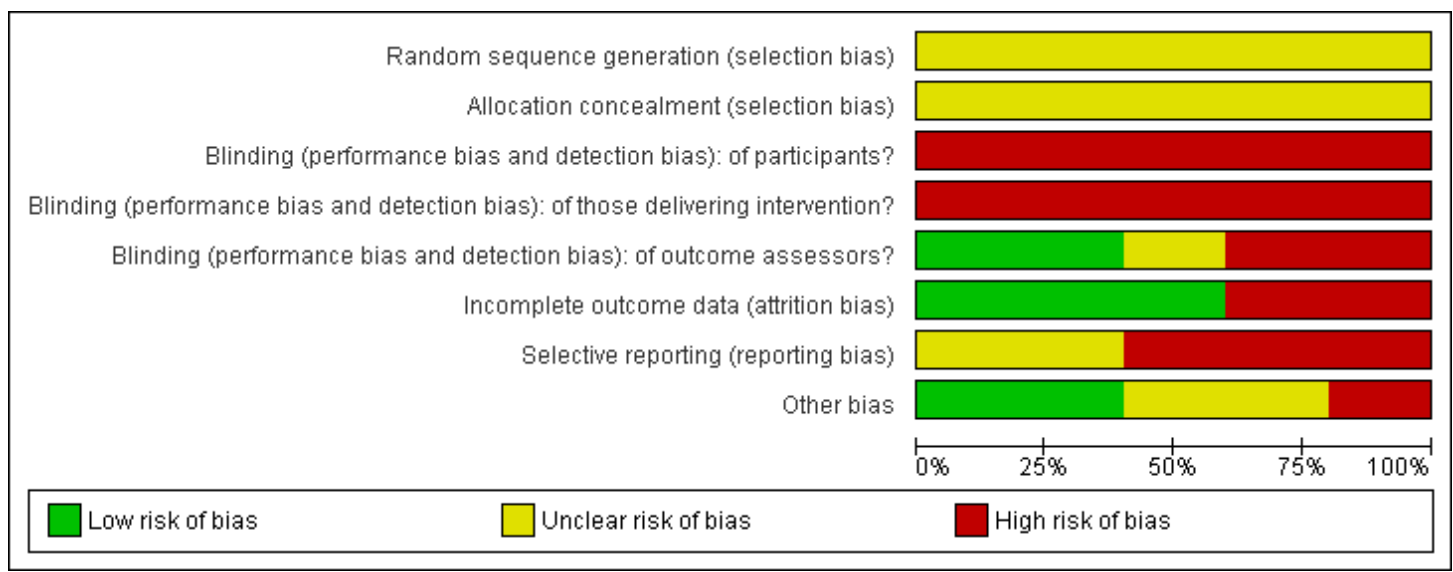

\section{Allocation}

All included studies were described by investigators as randomised, and the majority as stratified or block designs (see above). No study included in this review described the precise method by which a sequence was generated or allocation concealed. Therefore, overall the risk of bias for this criterion is unclear.

\section{Blinding}

As is common with psychological interventions, especially where outcomes are self-reported, there is inherent bias given the impossibility of blinding participants or those delivering interventions to treatment status. For this reason, despite the best intentions of researchers, the overall risk of bias in all studies is high. Where outcomes are not self-reported, outcome assessors can and should be kept blind.

We rated two studies as having high risk of bias (Fallone 1998; Lehner-Dua 2001); two as being unclear (van den Hoofdakker 2007; Mikami 2010); and one as being at low risk of bias (Blakemore 1993).

\section{Incomplete outcome data}

Most studies lost only a few participants. Two studies reported using intention-to-treat analysis (van den Hoofdakker 2007; Mikami 2010). Attrition was uneven in Lehner-Dua 2001.

\section{Selective reporting}

Original trial protocols were not available for any study in this review. Data for all reasonable outcomes for each study appear to have been reported. Remaining concerns include the fact that Blakemore 1993 only ever published preliminary results, in graph format without tables, and van den Hoofdakker 2007 collected data for both parents but only "analyzed the data from the mothers” (p.266) (van den Hoofdakker 2007). Fallone 1998 clearly collected data for all outcomes but did not report them all numerically, even in additional tables, concentrating mostly on the presentation of significant results. The overall judgment for included studies remains as unclear risk of bias.

\section{Other potential sources of bias}

Chief sources of other bias include issues of potential contamination in the study conducted by Lehner-Dua 2001, where the primary investigator led both parent training and control conditions.

\section{Effects of interventions}

In the protocol for this review, the following comparisons were planned:

- parent training versus a no treatment or waitlist control;

- parent training versus routine care (treatment as usual);

- parent training versus unstructured group parent support meetings. 
As described above, given that child participants in all studies remained on pre-existing treatment regimes and that control groups did not differ substantially, results for all studies are reported together. Opportunities for meta-analysis were, however, very limited due to issues of presentation of outcome data, so the majority of results are reported narratively.

\section{Change in the child's ADHD symptom-related behaviour in home setting}

The ADHD Index subscale of the Conners' Parent Rating ScaleRevised Short Form (CPRS-R-S) (Conners 2001) was used by van den Hoofdakker 2007. Within this 27 item scale, a higher number indicates greater psychopathology. Fallone 1998 employed the ADDES-Home (McCarney 1995) to measure this construct, wherein a higher value indicates less severity. Pooling results for van den Hoofdakker 2007 and Fallone 1998 for this outcome was not possible, even using standardised mean difference and compensating for opposing directions of scales, as the ADDES scale is divided into two subscales (the Hyperactive-Impulsive scale and the ADDES-Inattentive scale) (McCarney 1995) but is not a combined measure. In addition, van den Hoofdakker 2007 reported endpoint data and Fallone 1998 reported only change data in full (means and standard deviations). Means without standard deviations (SD) are supplied, but not for the entire sample: we calculated whole sample SDs using standard errors of within-group differences and report all data narratively below.

\section{Narrative reports of results}

Using the CPRS, van den Hoofdakker 2007 ( $n=62$ ) reported that, with respect to the ADHD Index of the CPRS, both groups (intervention plus routine clinical care (RCC) and RCC alone) improved, but that adjunctive parent training was not significantly better than with RCC alone. Results for the intervention group were: $19.0(\mathrm{SD}=6.2)$ versus $18.7(\mathrm{SD}=7.7)$ for the $\mathrm{RCC}$ control group.

On the ADDES-Home's Hyperactive-Impulsive scale, Fallone 1998 reported that both the parent training (PT) and enhanced parent training treatment (PT and self-management $(\mathrm{SM})$ ) groups did rate significantly better than control (mean of PT alone $=$ 7.50 (calculated SD = 1.64); mean of PT and SM group $=7.46$ (calculated SD $=1.64)$; mean of control group $=5.69$ (calculated $\mathrm{SD}=1.84)$ ).

On the ADDES-Home's Inattentive scale, Fallone 1998 reported that both the parent training and enhanced parent training treatment groups did significantly better than control (mean of PT alone $=6.43$ (calculated SD $=1.44)$; mean of $\mathrm{PT}$ and $\mathrm{SM}$ group $=7.31$ (calculated SD = 1.96); mean of control group = 4.50 (calculated $\mathrm{SD}=1.92)$ ).

\section{Change in the child's ADHD symptom-related behaviour in school setting}

No meta-analysis was possible for this outcome as the two studies that assessed it (Fallone 1998; Mikami 2010) reported it in very different ways. Fallone used both Achenbach 1991's Child Behaviour Checklist's Teacher Report Form (TRF) and McCarney 1995's ADDES-School measures. The TRF includes a Total Problems and a Total Externalizing and Total Internalizing scale (see note below), whereas the ADDES-School comprises Inattentive and Hyperactive-Impulsive scales. Fallone 1998 reported no numerical data for either of these instruments. Mikami 2010 reported results for the Teacher Questionnaire Measures of child social functioning (SSRS) and the teacher-assessed Dishion Social Acceptance Scale (DSAS) (Dishion 2003) to consider children's progress in this domain. Mikami reported data in the form of means and SDs.

\section{Narrative reports of results of included studies}

Fallone reported only that "analysis of teacher ratings failed to identify significant differences between treatment and control subjects from baseline to post-treatment. No significant changes on TRF and ADDES-School scales were detected for any group" ( $p$ 37) (Fallone 1998). No data were reported in tables. As above, the review authors await information that will assist in reporting numerical data for this outcome.

\section{Changes in the child's general behaviour}

As described above, problem behaviours are viewed in two broad dimensions. Externalising problems include conflict with others, whereas internalising problems reflect dealing with stress internally. Externalizing and Internalizing subscales of the Child Behaviour Checklist were used by van den Hoofdakker 2007 (Dutch version) (Achenbach 1991) and by Fallone 1998 (Achenbach 1986). Externalizing and Internalizing subscales of the Behaviour Assessment System for Children (BASC) (Reynolds 1998) were used by Lehner-Dua 2001.

The Eyberg Child Behaviour Inventory (ECBI) (Eyberg 1999) was used by investigators in Blakemore 1993.

Mikami 2010 (a study where the focus was on children's social development) used the Social Skills Rating System (SSRS) (Gresham 1990).

\section{Results from meta-analysis}

\section{Externalising}

Meta-analysis of three studies (Fallone 1998; Lehner-Dua 2001; van den Hoofdakker 2007) ( $\mathrm{n}=190)$ yielded results in favour of the intervention group, but fall short of significance (SMD -0.32; $95 \%$ CI -0.83 to $\left.0.18, \mathrm{I}^{2}=60 \%\right)$. 


\section{Internalising}

Results from meta-analysis of two studies (Lehner-Dua 2001; van den Hoofdakker 2007) ( $\mathrm{n}=142)$ yielded significant results in favour of the intervention group (SMD - $0.48 ; 95 \%$ CI -0.84 to $\left.0.13, \mathrm{I}^{2}=9 \%\right)$.

Fallone 1998 appeared to have collected, but not reported, numerical data for the Internalising subscale of the CBCL instrument.

\section{Narrative reports of results of included studies}

The Eyberg Child Behaviour Inventory (ECBI) (Eyberg 1999) was used by Blakemore 1993 ( $\mathrm{n}=24)$. Means are not supplied in the text or any table but they are supplied separately for mothers and fathers plotted on a graph that is difficult to interpret. No SDs are given. The investigators' own interpretation is as follows: "According to this measure (Figure 2) there were significant declines for mothers in the frequency of problem behaviours for both individual and group treatment relative to controls, although the effects were stronger for individual [treatment]. Data for fathers indicated a significant decline in the frequency of problem behaviours only for individual treatment relative to controls" (p 81). The investigators add that "effects for group treatment in fathers were not possible to evaluate because of large pre-treatment differences relative to both individual and control treatments". Investigators summarised their data as follows: "there appears to be stronger treatment effects for individual than group treatment and stronger effects for mothers than fathers" and that the former persist at follow-up and that the latter do not.

Investigators from the Mikami 2010 study $(\mathrm{n}=62)$ reported results for the SSRS for the intervention group (parental friendship coaching $(\mathrm{PFC})$ ) as $90.86(\mathrm{SD}=14.68)$ versus $83.87(\mathrm{SD}=16.28)$ in the control group. After accounting for "demographic covariates and baseline parent reports on the SSRS", there was a "between small and medium" effect size between intervention and control, and no interactions appeared to exist between treatment and sex, medication or oppositional defiant disorder (ODD) status.

Investigators from Mikami $2010(\mathrm{n}=62)$ (wherein the target of the intervention was children's social development) reported results for the SSRS (Teacher Questionnaire) for the intervention group $(\mathrm{PFC})$ as $92.38(\mathrm{SD}=12.90)$ versus $86.45(\mathrm{SD}=10.92)$ in the control group. The investigators reported that this difference was not significant; however, there was an interaction between treatment and ODD status. This indicated that the effect on teacher reports of child social skills may have been generally positive for the children without ODD who had received PFC (beta $=0.16$; $\mathrm{P}=-0.04$ ) but was not significant for those with ODD (beta = $0.09 ; \mathrm{P}=0.47$ ).

Investigators from Mikami 2010 ( $n=62)$ also reported results for the DSAS like or accept (teacher assessed) for the intervention group $(\mathrm{PFC})$ as $3.45(\mathrm{SD}=1.35)$ versus $2.62(\mathrm{SD}=1.15)$ in the control group. This outcome referred to the number of classroom peers who "like and accept" the child. Effect sizes were "between small and medium" and there were no interactions for this dependent variable. Results for the DSAS dislike or reject (teacher assessed) for the intervention group (PFC) were reported as 1.66 $(\mathrm{SD}=0.81)$ versus 2.21 ( $\mathrm{SD}=1.11)$ in the control group. Effect sizes were "between small and medium" and interactions appeared to include a positive effect of PFC for girls relative to boys and for medicated youth relative to nonmedicated youth.

\section{Academic achievement measured through school test results}

No study reported on this outcome.

\section{Adverse events}

No study reported adverse events in any way.

\section{Changes in parenting skills}

Lehner-Dua 2001 used the Parenting Sense of Competence (PSOC) (Johnston 1989) to assess whether the parent training programme "would significantly increase parents' sense of competence in comparison to a support group". The investigator reported significant improvement in both groups on the PSOC. The parent training group improved from a score of 58.48 (SD $=12.53)$ to $70.17(\mathrm{SD}=8.99)$, whilst the parent support group changed from $55.2(\mathrm{SD}=13.24)$ to $59.08(\mathrm{SD}=11.63)(\mathrm{P}<0.01)$ (MD 11.09; 95\% CI 5.23 to 16.95). The author commented that because of the absence of a 'no contact' group, "the mechanisms for these meaningful changes cannot be ascertained”.

\section{Parental stress}

Blakemore 1993, Lehner-Dua 2001 and van den Hoofdakker 2007 all used a variant of the Parenting Stress Index (PSI) (Abidin 1986) or short form (PSI-SF) (Abidin 1990) for this outcome; Fallone 1998 measured parental stress using the Revised Symptom Checklist (SCL-90-R) (Derogatis 1994).

\section{Results from meta-analysis}

Meta-analysis involving two studies (Lehner-Dua 2001; van den Hoofdakker 2007) (combined $n=142$ ) gave results for both the Parent and Child Domains of the PSI instrument (Child Domain Stress is linked to the parent's perception of the child's behaviour and Parent Domain Stress is a more general measure).

Results of meta-analysis of data from these two studies indicated no statistically significant difference between parent training and control for the Parent Domain (PD) of the PSI (MD -7.54; $95 \%$ CI -24.38 to $\left.9.30, \mathrm{I}^{2}=34 \%\right)$. Results for the PSI Child Domain (CD), however, were significant in favour of the intervention group (MD $-10.52 ; 95 \% \mathrm{CI}-20.55$ to $-0.48, \mathrm{I}^{2}=0 \%$ ). 


\section{Narrative results}

Investigators from the Blakemore 1993 study $(\mathrm{n}=24)$ also reported PSI total score data in the form of means plotted on a graph or figure, as described above, with no numerical data in tables or text. They reported, however, "an advantage for parents participating in the individual therapy program" and that these gains were durable and even increased at follow-up, at least for mothers. Subscales of the PSI including the Child Domain Stress and Parent Domain Stress were also reported as improving significantly for mothers but not for fathers. In contrast, their data suggested that fathers benefit more from group treatment, but that these benefits did not last.

Fallone 1998 reported change data for within group results for parental stress using a global measure for this outcome, the SCL90-R (Derogatis 1994). Means without SDs were reported for endpoint data; we calculated the SDs by using within-group standard errors, as recommended by the Cochrane Handbook for Systematic Reviews of Interventions (Deeks 2008). Results were: mean $=47.86(\mathrm{SD}=8.23)$ for the parent training only group; for the parent training plus self management group, mean $=53.31(\mathrm{SD}=$ 6.78); for the control group, mean $=55.56(\mathrm{SD}=5.08)$. Fallone reported these differences to be significant in favour of the parent training group $(\mathrm{P} \leq 0.01)$ but not for any other group.

\section{Parental understanding of ADHD}

No study included in this review measured this outcome specifically.

\section{DISCUSSION}

\section{Summary of main results}

Studies were few, small and disparate in focus. Opportunities for meta-analysis were limited due to issues of presentation of outcome data and we reported the majority of results narratively.

\section{Change in the child's ADHD-symptom-related behaviour in the home setting}

Two studies addressed this. The findings of the largest study in this review (van den Hoofdakker 2007) suggested that both groups improved but parent training plus routine clinical care was not significantly better than routine clinical care alone. Fallone 1998 reported that both parent training and enhanced parent training were significantly better than a waitlist control.

\section{Change in the child's ADHD-symptom-related behaviour in the school setting}

Two studies reported on this outcome. One yielded no numerical data, stating only that the results were not significant (Fallone 1998). They attributed this to the fact that most children in the study were taking medication that was most effective during school hours. The second study (Mikami 2010) focused mainly on social interaction outcomes for the children. Mikami 2010 concluded that in this domain (Teacher Questionnaire) parent training was only significantly better where ODD was not comorbid with ADHD.

Mikami 2010 also reported teacher assessments of whether children with ADHD were liked and accepted by their peers. Effect sizes were between "small and medium" for both outcomes. There was more effect in girls relative to boys and in medicated relative to nonmedicated participants.

\section{Changes in the child's general behaviour}

A meta-analysis of three studies (Fallone 1998; Lehner-Dua 2001; van den Hoofdakker 2007) ( $n=190)$ favours parent training. However, the effect did not reach significance (SMD - 0.27; 95\% CI -0.57 to $\left.0.03, \mathrm{I}^{2}=60 \%\right)$. Results from meta-analysis of two studies (Lehner-Dua 2001; van den Hoofdakker 2007) ( $\mathrm{n}=142$ ) yields significant results in favour of the parent training (SMD $0.48 ; 95 \%$ CI -0.81 to $-0.14, \mathrm{I}^{2}=9 \%$ ) for internalising behaviour. We had concerns about selective outcome reporting for a third study (Fallone 1998), which could have contributed data to this outcome but did not.

The Eyberg Child Behaviour Inventory (ECBI) (Eyberg 1999) was used by Blakemore 1993 ( $\mathrm{n}=24$ ). Investigators summarise their data as follows: "There appears to be stronger treatment effects for individual than group treatment and stronger effects for mothers than fathers" and the former persisted at follow-up and the latter did not.

Investigators from the Mikami 2010 study ( $\mathrm{n}=62$ ) reported results for the SSRS for the intervention group (PFC) as 90.86 (SD $=14.68)$ versus $83.87(\mathrm{SD}=16.28)$ in the control group. After accounting for "demographic covariates and baseline parent reports on the SSRS", there was a "between small and medium" effect size between the intervention and control; no interactions appeared to exist between treatment and sex, medication or ODD status.

\section{Changes in parenting skills}

Lehner-Dua 2001 used the Parenting Sense of Competence (PSOC) (Johnston 1989) to assess whether the parent training programme "would significantly increase parents' sense of competence in comparison to a support group". There was significant improvement in both groups on the PSOC. The author commented that because of the absence of a 'no contact' group, "the mechanisms for these meaningful changes cannot be ascertained". 


\section{Parental stress}

Meta-analysis involving two studies (Lehner-Dua 2001; van den Hoofdakker 2007) (combined $n=142$ ) gave results for both the Parent and Child Domains of the PSI instrument. Results were significant for the Child Domain but not for the Parent Domain. Blakemore 1993 ( $n=24$ ) reported "an advantage for parents participating in the individual therapy program"; these gains were durable and even increased at follow-up for mothers. In contrast, their data suggest that fathers benefit more from group treatment but that these benefits do not last. Fallone 1998 reported change data for within group results for parental stress using a global measure for this outcome, the SCL-90-R (Derogatis 1994). Fallone 1998 reported differences to be significant in favour of the parent training group $(\mathrm{P} \leq 0.01)$ but not so for either the enhanced parent training group or for the control group.

No study reported outcome data related to:

- academic achievement measured through school test results;

- adverse events;

- parental understanding of ADHD.

\section{Overall completeness and applicability of evidence}

We found few trials that examined whether parent training reduced ADHD symptoms and associated difficulties in children. These generally supported the use of parent training, as is used in current practice, especially in improving outcomes related to the child's general behaviour. However, the overall quality of the studies was poor. Most were small and took place in developed countries (the majority in North America); they did not all examine outcomes linked to core problems of the child's ADHD and had limited post-intervention follow-up. Selective reporting of trial outcomes was a problem in this review. Obtaining data where it was missing or incomplete was not possible in all cases despite efforts to contact authors. Some were untraceable, especially where the studies were completed some time ago (for example, Blakemore 1993) and where it was essential to supplement the limited published graphical data. Exact details on medication regimes of the children within studies were not invariably explicit. On a more positive note, all included studies gave clear, detailed accounts of the parent training interventions, including the content, duration and frequency of sessions.

\section{Quality of the evidence}

This systematic review found limited evidence of sufficient methodological rigour and with adequate reporting to confidently assess the clinical effects of parent training interventions for children with ADHD. Overall the trials were few in number (five), small in size (numbers ranged from 24 to 96), and provided little information about core issues to allow us to assess risk of bias (such as methods of sequence generation, allocation concealment and completeness of outcome data). With the best intentions of investigators, some of the risks of bias in this review, for example blinding, cannot be overcome in the context of a trial delivering a psychosocial intervention that is then primarily assessed by the recipient of that intervention (a parent). Other methodological weaknesses are, however, in the control of investigators. These include the duty to report all outcome data consistently and fully and not, for example, only to do so if a significant positive result was achieved; or only to report data from one set of observers (for example, mothers and not fathers) or to fail to assess treatment fidelity (a key measure of whether even a successful programme can be 'rolled out' in the wider world). The extent to which a Cochrane review can draw conclusions about the effects of an intervention depends on whether the data and results from the included studies are valid. In particular, invalid studies may produce misleading results (Higgins 2008a). Overall, the internal validity of studies in this review has limitations.

\section{Potential biases in the review process}

Clinical work with children or young people with ADHD usually involves seeing both the identified patient and their family. In order to ascertain the effect of the parent training component of this intervention, we restricted inclusion criteria to trials in which there was no direct intervention involving the child or young person. We therefore excluded trials such as the MTA 1999, which included a parent training component but also involved direct work with the children. Similarly, we excluded trials of parent training in children with antisocial behaviour or disruptive behaviour disorders if the trials were not primarily focused on ADHD. This may therefore narrow the generalisability of the results of this review when considering interventions for children with comorbid conditions.

Our inclusion criteria also required participants for whom "the main problem is ADHD" and where the diagnoses "should be clinical diagnoses by specialists" (as opposed to trained or untrained researchers using instruments such as semi-structured interviews). Had inclusion criteria been less restrictive, we might have been able to include subsets of children from trials where the main focus of the trial was antisocial or disruptive behaviour rather than ADHD.

However, without the main focus being on ADHD, the children in subsets of data obtained from these trials would not have been typical of children with ADHD. Two excluded trials illustrate this in opposite ways. Scott 2001a excluded the more severe children with "hyperkinetic syndrome" at recruitment, yet later found that "a good half" of their participants nevertheless met the diagnostic criteria for ADHD (Scott 2011). The primary outcome of this trial was antisocial behaviour and by excluding the more severe cases, those that remained no longer reflected the wider spectrum of ADHD (even those who had attempted to 'manage' the condi- 
tion). On the other hand, a trial by Scahill 2006 involving children with disruptive behaviour and tics included 10 children with $\mathrm{ADHD}$, all receiving stimulant medication (and indeed, those with "untreated ADHD" had no chance of entering the trial). Had we included these trial subsets, whichever trial had the larger number of identified ADHD cases may have biased the ADHD sample in terms of severity and co-intervention.

As the primary focus of this review was the effects of parent training on change in child symptoms and behaviour, we restricted primary outcomes to child outcomes rather than parent outcomes. We excluded studies with parent outcomes only (for example, reduction in parental stress), which may have introduced some bias to this review. However, only three such trials were excluded (Corkum 1999; Odom 1996; Treacy 2005). Although Treacy 2005 could have added weight to our finding that parent training reduces parental stress, the loss of usable data from the three trials overall was small.

We may have failed to identify small studies because of a degree of publishing bias (Egger 1997) operating in this review, but do not think we would not have found large relevant studies. The low number of studies in this review is likely to be an artefact of investigators turning increasingly to 'add on' treatments, which do not meet our inclusion criteria, or the lack of inclusion of a no treatment control. For example, in Fabiano 2006 the primary investigator wished to have a no treatment control but was not permitted to as the ethics committee was so convinced of the effectiveness of parent training that it rejected a plea by a funded investigator to incorporate a no-treatment control arm in the RCT.

\section{Agreements and disagreements with other studies or reviews}

NICE recommend parent training as a treatment intervention in ADHD (NICE 2008) based on evidence from children under 12 years with oppositional defiant disorder (ODD) and conduct disorder (CD) (NICE 2006a). Their review did not, however, examine the evidence on parent training in children with ADHD specifically. We are not able to firmly support their recommendation based on the trials we examined. We found some evidence, though not strong, to support parent training in ADHD.

Miller 1998 found little evidence to support the use of psychosocial interventions in ADHD whereas Pelham 2008 suggests that a number of psychosocial interventions are effective.

The narrative review by Modesto-Lowe 2008 discusses studies on parenting children in $\mathrm{ADHD}$, including the co-occurrence of $\mathrm{ADHD}$ and childhood disruptive disorders, psychiatric disorders in parents of children with ADHD, and optimising parental-child interactions. They suggest that there may be "high levels of child rearing stressors induced by the child's ADHD symptoms" on parents, with the focus of the studies mainly being on mothers and sons. Results of this review confirm that parent training appears to reduce parental stress.
Modesto-Lowe 2008 also mentions that "the degree of parental dysfunction appears to correlate with the presence and severity of ADHD-related disruptive disorders such as ODD and CD" and that there "is evidence to suggest that poor parental skills may exacerbate children's self-control deficits and contribute to the development of additional disruptive disorders that worsen ADHD outcomes". A study by Sonuga-Barke 2002 similarly found that treatment of parental ADHD might be necessary to ensure effectiveness of parent training. The ADHD symptoms of children of parents without ADHD improved following parent training compared to the lack of ADHD symptom improvement in children of mothers with ADHD symptoms. This issue was not addressed by any of the studies included in this review.

Modesto-Lowe 2008 also states that "early treatment, positive parenting and the absence of comorbidity may all optimize functioning and likely improve the course of the disorder". We only looked at school-aged children, which may have excluded very early treatment. None of the included studies directly addressed this issue.

\section{AUTHORS' CONCLUSIONS}

\section{Implications for practice}

There is some indication that parent training may have a positive effect on difficulties experienced by children with ADHD, particularly in terms of general behaviour. Data are more encouraging for the parents and carers of such children (in whom parent training may well be of benefit in reducing parental stress and building a sense of parental confidence). However, data concerning ADHDspecific behaviour are more ambiguous. The poor methodological quality of the studies overall makes it likely that there is bias in the results and weakens any conclusions that may be drawn in this review. For many important outcomes, including school achievement and adverse effects, data for this intervention are lacking.

Overall, data from this review do not provide sufficiently strong evidence on which to base recommendations for practice.

\section{Implications for research}

Further well-designed, randomised controlled trials within this population are needed and should be reported clearly in accordance with the principles set out in the CONSORT 2010 Statement (www.consort-statement.org/consort-statement/). Measurement of treatment outcome is often limited to parent and teacher completed questionnaires and could be extended to include, for example, health-related quality of life outcomes (HRQL) (Klassen 2004). Trials need to collect information about adverse events related to any intervention. Researchers should consider child outcomes and not only focus on reduction of parental stress or sense of competence. Child outcomes may also include HRQL

Parent training interventions for Attention Deficit Hyperactivity Disorder (ADHD) in children aged 5 to 18 years (Review) 
(Klassen 2004), social interactions with peers, family interactions and school achievement.

As comorbidity is so common in $\mathrm{ADHD}$, further research with children displaying disruptive behaviour disorders should address these comorbid conditions and not focus on only one area, for example, ADHD or ODD/CD. The effects of gender, both that of the parent and child, should be carefully considered. Many parents and young people wish to limit the exposure of children to psychoactive medication, so it may be useful to explore whether this might be achieved through psychosocial interventions targeted at those most likely to benefit from them.

Furthermore, a complementary review of parent training for parents of children under the age of five years who have been assessed as 'at risk' of ADHD would be a timely addition to literature in this area.

\section{ACKNOWLEDGEMENTS}

We would like to thank persons formerly on the authorline of this protocol who made contributions at the early stages: Carol Joughin, Sima Pindoria (UK). Thanks also to members of the original steering group at the Systematic Reviews Training Unit, Institute of Child Health, University College London (UK), especially Stuart Logan.
Thanks also to Geraldine Macdonald (Co-ordinating Editor, CDPLPG) for her hard work and support of this project; to Jo Abbott (former Trials Search Co-ordinator of the Cochrane Developmental, Psychosocial and Learning Problems Group (CDPLPG)) for searches, and to anonymous statistical editors within the Cochrane and Campbell Collaborations. We are also grateful to Krystyna Kowalski (SFI Campbell, Denmark) for her patience and support over several years and to Cathy Bennett (Systematic Research Ltd, UK) for continuing assistance and attention to detail.

We would also like to thank those who helped us in obtaining potential studies for the review, including: Julie Millener (formerly of the CDPLPG editorial base), Yanina Sguassero (CDPLPG author, Argentina) and Jutta Stoffers (CDPLPG author, Germany). Thanks also to Angela Huertas-Ceballos (CDPLPG author, UK), Toby Lasserson of the Cochrane Airways Group (UK), Danielle Ouwejan of the University of Bristol (UK) and Dr Qihua Ye (People's Republic of China), who provided assistance with translations of potential studies from Spanish, German, Dutch and Chinese, respectively.

We would like to thank numerous primary investigators who assisted us in providing clarification on aspects of methodology and data from studies cited within this review. They include Dr Michelle Beyer (USA), Dr Anil Chacko (USA), Dr Richard Conte (USA), Dr Maj Britt Drugli and Dr Sturla Fossum (Norway), Dr Chauntel Gustis (USA), Professor Nick Ialongo (USA), Dr Sue Odom (USA), Professor Lawrence Scahill (USA) and Professor Stephen Scott (UK).

\section{RE F E R E N C E S}

\section{References to studies included in this review}

Blakemore 1993 \{published data only\}

Blakemore B, Shindler S. A problem solving training program for parents of children with attention deficit hyperactivity disorder. Canadian Journal of School Psychology 1993;9(1/Special Issue):66-85.

Fallone 1998 \{published data only\}

Fallone GP. Treatment for Maternal Distress as an Adjunct to Parent-Training for Children with Attention-Deficit Hyperactivity Disorder. Memphis TN: University of Memphis, 1998.

Lehner-Dua 2001 \{published data only\} Lehner-Dua LL. The Effectiveness of Russell A Barkley's Parent Training Program on Parents with School-Aged Children who have ADHD on their Perceived Severity of ADHD, Stress, and Sense of Competence (PhD dissertation). Hempstead, NY: Hofstra University, 2001.

Mikami 2010 \{published data only\}

Mikami AY, Jack A, Emeh CC, Stephens HF. Parental influence on children with attention-deficit/hyperactivity disorder: I. Relationships between parent behaviors and child peer status. Journal of Abnormal Child Psychology 2010;38(6):721-36.

* Mikami AY, Lerner MD, Griggs MS, McGrath A, Calhoun CD. Parental influence on children with attention-deficit/ hyperactivity disorder: II. Results of a pilot intervention training parents as friendship coaches for children. Journal of Abnormal Child Psychology 2010;38:737-49.

van den Hoofdakker 2007 \{published data only\} van den Hoofdakker BJ, van der Veen-Mulders L, Sytema S, Emmelkamp PM, Minderaa RB, Nauta MH. Effectiveness of behavioral parent training for children with $\mathrm{ADHD}$ in routine clinical practice: a randomized controlled study. Journal of the American Academy of Child and Adolescent Psychiatry 2007;46(10):1263-71.

\section{References to studies excluded from this review}

\section{Abikoff 2004 \{published data only\}}

Abikoff HB, Hechtman L, Klein RG, Gallagher R, Fleiss K, Etcovitch J, et al. Multimodal therapy and stimulants in the treatment of children with attention deficit hyperactivity disorder. Journal of the American Academy of Child and Adolescent Psychiatry 2004;43(7):820-9. 
Aman 2010 \{published data only\}

* Aman MG, Mcdougle CJ, Scahill L, Handen B, Arnold LE, Johnson C, et al. Medication and parent training in children with pervasive developmental disorders and serious behavior problems: results from a randomized clinical trial. Journal of the American Academy of Child and Adolescent Psychiatry 2009;48(12):1143-54.

Anastopoulos 1993 \{published data only\} Anastopoulos AD, Shelton TL, DuPaul GJ, Guevremont DC. Parent training for attention-deficit hyperactivity disorder: its impact on parent functioning. Journal of Abnormal Child Psychology 1993;21(5):581-96.

Arnold 2007 \{published data only\} Arnold SA. Positive Behavior Interventions for Adolescents with ADD/ADHD (Masters' thesis). Long Beach, CA: Callifornia State University, 2007.

Baker-Ericzen 2010 \{published data only\} Baker-Ericzen MJ, Hurlburt MS, Brookman-Frazee L, Jenkins MM, Hough RL. Comparing child, parent, and family characteristics in usual care and empirically supported treatment research samples for children with disruptive behavior disorders. Journal of Emotional and Behavioral Disorders 2010;18(2):82-99.

Bandsma 1997 \{published data only\} Bandsma LG. Tailor-made group mediation therapy for parents of children with attention-deficit/hyperactivity disorder. Evaluation of a therapeutic intervention: results and efficacy [Dutch] [Groepsmediatietherapie op maat voor ouders van ADHD-kinderen]. Kind En Adolescent 1997;18 (2):73-84.

Barkley 1992 \{published data only\} Barkley RA, Guevremont DC, Anastopoulos AD, Fletcher KE. A comparison of three family therapy programs for treating family conflicts in adolescents with attention-deficit hyperactivity disorder. Journal of Consulting and Clinical Psychology 1992;60(3):450-62.

Barkley 2000 \{published data only\} Barkley RA, Shelton TL, Crosswait C, Moorehouse M, Fletcher K, Barrett $S$, et al. Multi-method psychoeducational intervention for preschool children with disruptive behavior: preliminary results at post-treatment. Journal of Child Psychology and Psychiatry and Allied Disciplines 2000;41(3):319-32.

Barkley 2001 \{published data only\} Barkley RA, Edwards G, Laneri M, Fletcher K, Metevia L. The efficacy of problem-solving communication training alone, behavior management training alone, and their combination for parent-adolescent conflict in teenagers with ADHD and ODD. Journal of Consulting and Clinical Psychology 2001;69(6):926-41.

Barkley 2002b \{published data only\} Barkley RA. Preschool children with disruptive behavior: three-year outcome as a function of adaptive disability. Development and Psychopathology 2002;14(1):45-67.
Beyer 1994 \{published and unpublished data\}

Beyer MM. Group Parent-Training for Attention Deficit Hyperactivity Disorder (PhD dissertation). Ames, Iowa: Iowa State University, 1994.

\section{Bogle 2007 \{published data only\}}

* Bogle KE. Evaluation of a Brief Group Parent Training Intervention in the Context of an After-School Program for Middle School Students (PhD dissertation). Columbia, SC: University of South Carolina, 2007.

Bor 2002 \{published data only\} Bor W, Sanders MR, Markie Dadds C. The effects of the Tripple P-positive parenting programme on preschool children with co-occurring disruptive behaviour and attentional/hyperactive difficulties. Journal of Abnormal Child Psychology 2002;68(4):624-40.

Chacko 2007 \{published data only\}

* Chacko A. Treatment for Single Mothers of Children Diagnosed with ADHD: A Comparison Between a Traditional and an Enhanced Behavioural Parenting Programme (PhD dissertation). Buffalo, NY: State University of New York at Buffalo, 2007.

Chacko A, Wymbs BT, Wymbs FA, Pelham WE, SwangerGagne MS, Girio E, et al. Enhancing traditional behavioral parent training for single mothers of children with ADHD. Journal of Clinical Child and Adolescent Psychology 2009;38 (2):206-18.

Chronis 2004 \{published data only\}

Chronis AM, Fabiano GA, Gnagy EM, Onyango AN, Pelham Jr WE, Lopez Williams A, et al. An evaluation of the summer treatment program for children with attentiondeficit/hyperactivity disorder using a treatment withdrawal design. Behavior Therapy 2004;35(3):561-85.

Chronis 2006 \{published data only\} Chronis AM, Gamble SA, Roberts JE, Pelham WE. Cognitive-behavioral depression treatment for mothers of children with attention-deficit/hyperactivity disorder. Behavior Therapy 2006;37(2):143-58.

Connell 1997 \{published data only\} Connell S, Sanders MR, Markie-Dadds C. Self-directed behavioral family intervention for parents of oppositional children in rural and remote areas. Behavior Modification 1997;21(4):379-408.

\section{Corkum 1999 \{published data only\}} Corkum P, Rimer P, Schachar R. Parental knowledge of attention-deficit hyperactivity disorder and opinions of treatment options: impact on enrolment and adherence to a 12-month treatment trial. Canadian Journal of Psychiatry 1999;44(10):1043-8.

Schachar RJ, Tannock R, Cunningham C, Corkum PV. Behavioral, situational, and temporal effects of treatment of ADHD with methylphenidate. Journal of the American Academy of Child and Adolescent Psychiatry 1997;36(6): 754-63.

Corkum 2005 \{published data only\} Corkum PV, McKinnon MM, Mullane JC. The effect of involving classroom teachers in a parent training program 
for families of children with ADHD. Child and Family Behavior Therapy 2005;27(4):29-49.

Corrin 2003 \{published data only\}

Corrin EG. Child Group Training Versus Parent and Child Group Training for Young Children with ADHD (PhD dissertation). Madison, NJ: Fairleigh Dickinson University, 2003.

Coughlin 2009 \{published data only\}

Coughlin M, Sharry J, Fitzpatrick C, Guerin S, Drumm M. A controlled clinical evaluation of the parents plus children's programme: a video-based programme for parents of children aged 6 to 11 with behavioural and developmental problems. Clinical Child Psychology and Psychiatry 2009;14 (4):541-58.

Cummings 2008 \{published data only\} Cummings JG, Wittenberg JV. Supportive expressive therapy - parent child version: an explorative study. Psychotherapy: Theory, Research, Practice, Training 2008;45 (2):148-64.

Danforth 1998 \{published data only\}

Danforth JS. The outcome of parent training using the Behavior Management Flow Chart with mothers and their children with oppositional defiant disorder and attentiondeficit hyperactivity disorder. Behavior Modification 1998; 22(4):443-73.

\section{Dubbs 2008 \{published data only\}}

Dubbs JL. Parent stress reduction though a psychosocial intervention for children diagnosed with Attention-Deficitl Hyperactivity Disorder (PhD dissertation). Philadelphia, PA: University of Pennsylvania, 2008.

Dubey 1978 \{published data only\}

Dubey DR, Kaufman KF. Home management of hyperkinetic children. Journal of Pediatrics 1978;93(1): 141-6.

Dubey DR, O'Leary SG, Kaufman KF. Training parents of hyperactive children in child management: a comparative outcome study. Journal of Abnormal Child Psychology 1983; 11(2):229-45.

Ellis 2009 \{published data only\} Ellis B, Nigg J. Parenting practices and attention-deficit/ hyperactivity disorder: new findings suggest partial specificity of effects. Journal of the American Academy of Child and Adolescent Psychiatry 2009;48(2):146-54.

Ercan 2005 \{published data only\} Ercan EE, Dogan O, Dogan S, Sumer H. Effects of combined treatment on Turkish children diagnosed with attention-deficit/hyperactivity disorder: a preliminary report. Journal of Child and Adolescent Psychopharmacology 2005;15(2):203-19.

Fabiano 2006 \{published data only\}

* Fabiano GA. Behavioral Parent Training for Fathers of Children with Attention-Deficit/Hyperactivity Disorder: Effectiveness of the Intervention and a Comparison of Two
Formats [PhD dissertation]. Buffalo, NY: State University of New York at Buffalo, 2005.

Fabiano GA, Chacko A, Pelham Jr WE, Robb J, Walker KS, Wymbs F, et al. A comparison of behavioural parent training programmes for fathers of children with AttentionDeficit/Hyperactivity Disorder. Behavior Therapy 2009;40 (2):190-204.

Fagan Rogers 2009 \{published data only\} Fagan Rogers MA. The Role of Parental Involvement in the Learning of Children with Attention-Deficit/Hyperactivity Disorder (PhD dissertation). Toronto: University of Toronto, 2008.

Gibbs 2008 \{published data only\}

Gibbs A, Moor S, Frampton C, Watkins W. Impact of psychosocial interventions on children with disruptive and emotional disorders treated in a health camp. Australian and New Zealand Journal of Psychiatry 2008;42(9):789-99.

Grimm 2006 \{published data only\}

Grimm K, Mackowiak K. KES training for parents of children with conduct behaviour problems. Praxis der Kinderpsychologie und Kinderpsychiatrie 2006;55(5):363-83.

Guo 2008 \{published data only\}

Guo QF, Zhang YH, Zhang Z. Effect of family intervention on family environment and the parental rearing pattern of children with ADHD. Chinese Mental Health Journal 2008; 22:423-9.

Gustis 2007 \{published and unpublished data\} Gustis C. Behavioral Intervention and Parent Training within the CASSP System: The Effectiveness of Using Direct Commands to Manage ADHD/ODD in the Home Environment ( $P h D$ dissertation). Minneapolis, MN: Cappella University, 2007.

Hall 2003 \{published data only\} Hall TF. Early Intervention Multimodal Treatment Programme for Children with Attention Deficit Hyperactivity Disorder: An Outcome Study (Phd dissertation). Madison, NJ: Fairleigh Dickinson University, 2003.

Hauth-Charlier 2009 \{published data only\} Hauth-Charlier S, Clement C. Behavioral parent training programs for parents of children with ADHD: Practical considerations and clinical implications. Pratiques Psychologiques 2009;15(4):457-72.

Hautmann 2009 \{published data only\} Hautmann C, Stein P, Hanisch C, Eichelberger I, Pluck J, Walter D, et al. Does parent management training for children with externalizing problem behavior in routine care result in clinically significant changes?. Psychotherapy Research 2009;19(2):224-33.

\section{Heriot 2008 \{published data only\}} Heriot, SA, Evans IM, Foster TM. Critical influences affecting response to various treatments in young children with ADHD: a case series. Child: Care, Health and Development 2008;34(1):121-33.

Horn 1991 \{published data only\} Horn WF, Ialongo NS, Pascoe JM, Greenberg G, Packard T, Lopez M, et al. Addictive effects of psychostimulants, 
parent training and self-control therapy with ADHD children. Journal of the American Academy of Child and Adolescent Psychiatry 1991;30(2):233-40.

Isler 2003 \{published data only\}

Isler L. Effects of Gender on Treatment Outcome in Young Children with ADHD (PhD dissertation). Madison, NJ: Fairleigh Dickinson University, 2003.

Jones 2008 \{published data only\}

Jones K, Daley D, Hutchings J, Bywater T, Eames C. Efficiency of the Incredible Years Basic parent training programme as an early intervention for children with conduct problems and ADHD. Child: Care, Health and Development 2007;33(6):749-56.

\section{Larsson 2008 \{published data only\}}

Drugli MB, Larsson B. Children aged 4-8 years treated with parent training and child therapy because of conduct problems: generalisation effects to day care and school settings. European Child and Adolescent Psychiatry 2006;15 (7):392-7.

Fossum S. Email correspondence between Sturla Fossum and Jane Dennis detailing reasons why ADHD diagnosis was not 'secure' 2011 (3 February).

Fossum S, Mørch WT, Handegård BH, Drugli MB, Larsson B. Parent training for young Norwegian children with ODD and $\mathrm{CD}$ problems: predictors and mediators of treatment outcome. Scandinavian Journal of Psychology 2009;50(2): 173-81. DOI: 10.1111/j.1467-9450.2008.00700.x * Larsson B, Fossum S, Clifford G, Drugli MB, Handegård BH, Mørch WT. Treatment of oppositional defiant and conduct problems in young Norwegian children: results of a randomized controlled trial. European Child and Adolescent Psychiatry epublication 2008 Jun 18;18(1):42-52. DOI: 10.1007/s00787-008-0702-z

Mørch WT. The Incredible Years in Norway: the treatment of oppositional defiant and conduct problems in young Norwegian children (registered trial protocol). www.controlled-trials.com/ISRCTN10430476 (accessed 30 December 2010) 2008.

Lauth 2007 \{published data only\}

Lauth GW, Grimm K, Otte TA. Behavior training exercises for parents: a study of effectiveness [German] [Verhaltensubungen im elterntraining: Eine studie zur differenzierten wirksamkeit im elterntraining]. Zeitschrift fur Klinische Psychologie und Psychotherapie 2007;36(1): 26-35.

Lavigne 2008 \{published data only\}

Lavigne JV, LeBailly SA, Gouze KR, Cicchetti C, Pochyly J, Arend R, et al. Treating oppositional defiant disorder in primary care: a comparison of three models. Journal of Pediatric Psychology 2008;33(5):449-61. DOI: 10.1093/ jpepsy/jsm074

Markie-Dadds 2006 \{published data only\}

Markie-Dadds C, Sanders MR. A controlled evaluation of an enhanced self-directed behavioural family intervention for parents of children with conduct problems in rural and remote areas. Behaviour Change 2006;23(1):55-72.
McGoey 2005 \{published data only\}

McGoey KE, DuPaul GJ, Eckert TL, Volpe RJ, Van Brakle J. Outcomes of a multi-component intervention for preschool children at risk for attention-deficit/hyperactivity disorder. Child and Family Behavior Therapy 2005;27(1): $33-56$.

Miranda 2006 \{published data only\}

Miranda A, Jarque S, Rosel J. Treatment of children with ADHD: psychopedagogical programme at school versus psychostimulant medication. Psicothema 2006;18(3): 335-41.

Molina 2008 \{published data only\} Molina BSG, Flory K, Buckstein OG, Greiner AR, Baker JL, Krug V, et al. Feasibility and preliminary efficacy of an after-school programme for middle schoolers with ADGHD: a randomized trial in a large public middle school. Journal of Attention Disorders 2008;12(3):207-17.

Montiel 2002 \{published data only\}

Montiel Nava C, Pena JA, Espina Marines G, FerrerHernandez ME, López-Rubio A, Puertas-Sánchez S, et al. A pilot study of methylphenidate and parent training in the treatment of children with attention-deficit hyperactivity disorder [Estudio piloto de metilfenidato y entrenamiento a padres en el tratamiento de niños con trastorno por déficit de atenciÓn-hiperactividad [Spanish]]. Revista de Neurologia 2002;35(3):201-5.

Morawska 2009 \{published data only\}

Morawska A, Sanders M. An evaluation of a behavioural parenting intervention for parents of gifted children. Behaviour Research and Therapy 2009;47(6):463-70.

MTA 1999 \{published data only\}

Swanson J, Arnold LE, Kraemer H, Hechtman L, Molina B, Hinshaw $S$, et al. Evidence, interpretation and qualification from multiple reports of long-term outcomes in the multimodal treatment study of children with ADHD (MTA) Part II; supporting details. Journal of Attention Disorders 2008;12(1):15-43.

* The MTA Cooperative Group. A 14-month randomized clinical trial of treatment strategies for attention-deficit/ hyperactivity disorder. Archives of General Psychiatry 1999; 56(12):1073-86.

Wells KC, Pelham WE Jr, Kotkin RA, Hoza B, Abikoff HB, Abramowitz A, et al. Psychosocial treatment strategies in the MTA Study: rationale, methods, and critical issues in design and implementation. Journal of Abnormal Child Psychology 2000;28(6):483-505.

Nixon 2001 \{published data only\} Nixon RDV. Changes in hyperactivity and temperament in behaviourally disturbed preschoolers after parent-child interaction therapy (PCIT). Behaviour Change 2001;18(3): $168-76$.

Nixon 2003 \{published data only\}

Nixon RD, Sweeney L, Erickson DB, Touyz SW. Parentchild interaction therapy: a comparison of standard and abbreviated treatments for oppositional defiant preschoolers. 
Journal of Consulting and Clinical Psychology 2003;71(2): 251-60.

O'Leary 1976 \{published data only\}

O'Leary KD, Pelham WE, Rosenbaum A, Price GH. Behavioral treatment of hyperkinetic children. An experimental evaluation of its usefulness. Clinical Pediatrics 1976;15(6):510-15.

Odom 1996 \{published data only\}

Odom SE. Effects of an educational intervention on mothers of male children with attention deficit hyperactivity disorder. Journal of Community Health Nursing 1996;13(4): $207-20$.

Pisterman 1989 \{published data only\}

Pisterman S, Firestone P, McGrath P, Goodman JT, et al. The effects of parent training on parenting stress and sense of competence. Canadian Journal of Behavioural Science 1992;24(1):41-58.

* Pisterman S, McGrath P, Firestone P, Goodman JT, Webster I, Mallory R. Outcome of parent-mediated treatment of preschoolers with attention deficit disorder with hyperactivity. Journal of Consulting and Clinical Psychology 1989;57(5):628-35.

Pisterman 1992a \{published data only\}

Pisterman S, Firestone P, McGrath P, Goodman JT, Webster I, Mallory R, et al. The effects of parent training on parenting stress and sense of competence. Canadian Journal of Behavioural Science 1992;24(1):41-58.

* Pisterman S, Firestone P, McGrath P, Goodman JT, Webster I, Mallory R, et al. The role of parent training in treatment of preschoolers with ADDH. American Journal of Orthopsychiatry 1992;62(3):397-408.

Pollard 1983 \{published data only\}

Pollard S, Ward EM, Barkley RA. The effects of parent training and Ritalin on the parent-child interactions of hyperactive boys. Child and Family Behavior Therapy 1983; 5(4):51-69.

Reeves 2009 \{published data only\}

Reeves G, Anthony B. Multimodal treatments versus pharmacotherapy alone in children with psychiatric disorders: implications of access, effectiveness and contextual treatment. Paediatric Drugs 2009;11(3):165-9.

Salbach 2005 \{published data only\}

Salbach H, Lenz K, Huss M, Vogel R, Felsing D, Lehmukel $\mathrm{U}$. Treatment effects of parent management training for ADHD [Die Wirksamkeit eines Gruppentrainings fur Eltern hyperkinetischer Kinder [German]]. Zeitschrift fur Kinder- und Jugendpsychiatrie und Psychotherapie 2005;33 (1):59-68.

Sanders 2000a \{published data only\}

McLennan JD. Behavioural family therapy reduced disruptive behaviour in children at risk for developing conduct problems. Evidence Based Mental Health 2001;4 (1):20.

* Sanders MR, Markie Dadds C, Tully LA, Bor W. The Triple P-Positive Parenting Programme: a comparison of enhanced, standard and self directed behavioural family intervention for parents of children with early onset conduct problems. Journal of Consulting and Clinical Psychology 2000;68(4):624-40.

Sanders 2000b \{published data only\}

Sanders MR, McFarland M. Treatment of depressed mothers with disruptive children: A controlled evaluation of cognitive behavioural family intervention. Behaviour Therapy 2000;31(1):89-112.

Scahill 2006 \{published data only\}

Scahill L, Sukhodolsky DG, Bears K, Findley D, Hamrin V, Carroll DH, et al. Randomized trial of parent management training in children with tic disorders and disruptive behavior. Journal of Child Neurology 2006;21(8):650-6.

Schoppe-Sullivan 2009 \{published data only\} Schoppe-Sullivan SJ, Weldon AH, Cook JC, Davis EF, Buckley CK. Coparenting behavior moderates longitudinal relations between effortful control and preschool children's externalizing behavior. Journal of Child Psychology and Psychiatry and Allied Disciplines 2009;50(6):698-706.

Scott 2001a \{published data only\}

Scott S, Spender Q, Doolan M, Jacobs B, Aspland H. Multicentre controlled trial of parenting groups for childhood antisocial behaviour in clinical practice. $B M J$ 2001;323(7306):1-7.

Scott 2010 \{published data only\}

Scott S, Sylva K, Doolan M, Price J, Jacobs B, Crook C, et al. Randomised controlled trial of parent groups for child antisocial behaviour targetting multiple risk factors: the SPOKES Project. Journal of Child Psychology and Psychiatry and Allied Disciplines 2010;51(1):48-57.

Sonuga-Barke 2001 \{published data only\}

Baldwin L. Parent training improved maternal well being and reduced attention deficit hyperactivity disorder symptoms in children. Evidence-Based Nursing 2001;4(4): 109.

Sonuga Barke EJS, Daley D, Thompson M. Does maternal ADHD reduce the effectiveness of parent training for preschool children's ADHD?. Journal of the American Academy of Child and Adolescent Psychiatry 2002;41(6): 696-702.

* Sonuga Barke EJS, Daley D, Thompson M, Laver Bradbury C, Weeks A. Parent-based therapies for preschool attention-deficit/hyperactivity disorder: a randomized, controlled trial with a community sample. Journal of the American Academy of Child and Adolescent Psychiatry 2001; 40(4):402-8.

Sonuga-Barke 2004 \{published data only\} Sonuga Barke EJS, Thompson M, Daley D, Laver Bradbury C. Parent training for Attention Deficit/Hyperactivity Disorder: is it as effective when delivered as routine rather than as specialist care?. British Journal of Clinical Psychology 2004;43(4):449-57.

Springer 2004 \{published data only\}

Springer C. Treatment Adherence in an Early Intervention Programme for Children with Attention Deficit Hyperactivity 
Disorder (PhD dissertation). Madison, New Jersey: Fairleigh Dickinson University, 2004.

Taylor 1998 \{published data only\}

Taylor TK, Schmidt F, Pepler D, Hodgins C. A comparison of eclectic treatment with Webster-Stratton's parents and children series in a children's mental health centre: a randomized controlled trial. Behaviour Therapy 1998;29(2): $221-40$.

Treacy 2005 \{published data only\}

Treacy L, Tripp G, Baird A. Parent stress management training for attention-deficit/hyperactivity disorder. Behavior Therapy 2005;36(3):223-33.

van der Oord 2008 \{published data only\}

Coghill D. Adding multimodal behavioural therapy to methylphenidate does not improve ADHD outcomes. Evidence Based Mental Health 2007;10(4):124.

* van der Oord S, Prins PJM, Oosterlaan J, Emmelkamp PMG. Treatment of attention deficit hyperactivity disorder in children: predictors of treatment outcome. European Journal of Child and Adolescent Psychiatry 2008;17:73-81.

Waschbusch 2005 \{published data only\}

Waschbusch DA, Pelham WE Jr, Massetti G. The Behaviour Education Support and Treatment (BEST) school intervention program: pilot project data examining schoolwide, targeted-school and targeted-home approaches. Journal of Attention Disorders 2005;9(1):313-22.

Weinberg 1999 \{published data only\}

Weinberg HA. Parent training for attention deficit hyperactivity disorder: parental and child outcomes. Journal of Clinical Psychology 1999;55(7):907-13.

Wolraich 2005 \{published data only\}

Wolraich ML, Bickman L, Lambert EW, Simmons T, Doffing MA. Intervening to improve communication between parents, teachers, and primary care providers of children with ADHD or at high risk for ADHD. Journal of Attention Disorders 2005;9(1):354-68.

\section{Additional references}

\section{Abidin 1986}

Abidin RR. Parenting Stress Index. Odessa, FL: Psychological Assessment Resources, Inc, 1986.

\section{Abidin 1990}

Abidin RR. Parenting Stress Index. Revised. Odessa, FL: Psychological Assessment Resources, Inc, 1990.

\section{Abidin 1995}

Abidin RR. Parenting Stress Index. 3rd Edition. Odessa, FL: Psychological Assessment Resources, Inc, 1995.

\section{Achenbach 1986}

Achenbach TM, Edelbrock CS. Manual for the Child Behavior Checklist. Burlington, VT: University of Vermont, 1986.

\section{Achenbach 1991}

Achenbach TM. Manual for the Child Behavior Checklist 14-18 and 1991 profile. Burlington, VT: University of Vermont, 1991.

\section{American Academy of Pediatrics 2001}

American Academy of Pediatrics. Clinical Practice Guideline: treatment of the school-aged child with attention deficit hyperactivity disorder. Pediatrics 2001;108 (4): $1033-44$.

Anastopoulous 2009

Anastopoulous AD. Email to Jane Dennis concerning nonrandom assignment of participants to groups in 1993 paper published in 'Journal of Abnormal Child Psychology' 2009 (19 December).

\section{APA 1980}

American Psychiatric Association. Diagnostic and Statistical Manual of Mental Disorders, 3rd Edition (DSM III). Washington DC: American Psychiatric Association, 1980.

\section{APA 1987}

American Psychiatric Association. Diagnostic and Statistical Manual of Mental Disorders, 3rd edition - Revised (DSM-III$R$ ). Washington, DC: American Psychiatric Association, 1987.

\section{APA 1994}

American Psychiatric Association. Diagnostic and Statistical Manual of Mental Disorders, 4th edition (DSM-IV).

Washington DC: American Psychiatric Association, 1994

\section{Asherson 2007}

Asherson P, Brookes K, Franke B, Chen W, Gill M, Ebstein $\mathrm{RP}$, et al. Confirmation that a specific haplotype of the dopamine transporter gene Is associated with combinedtype ADHD. American Journal of Psychiatry 2007;164: 674-7.

\section{Barkley 1997}

Barkley RA. Defiant Children: A Clinician's Manual for Assessment and Parent Training. 2nd Edition. New York: Guildford Press, 1997.

\section{Barkley 2002a}

Barkley RA. International consensus statement on ADHD. Journal of the American Academy of Child and Adolescent Psychiatry 2002;41(12):1389.

Barlow 1997

Barlow J. Systematic review of the effectiveness of parenttraining programmes in improving the behaviour of 3-7 year old children. Oxford: Health Services Research Unit Report, 1997.

\section{Barlow 2010}

Barlow J, Parsons J. Group-based parent-training programmes for improving emotional and behavioural adjustment in 0-3 year old children. Cochrane Database of Systematic Reviews 2010, Issue 3. DOI: 10.1002/ 14651858.CD003680.pub2

\section{CDC 2005}

Center for Disease Control. Mental health in the United States: prevalence of diagnosis and medication treatment for attention-deficit/hyperactivity disorder - United States, 2003. MMWR Weekly (www.cdc.gov/mmwr/preview/ mmwrhtml/mm5434a2.htm) September 22005 (accessed 25 February 2009). 
Cheng 2007

Cheng JY, Chen RY, Ko JS, Ng EM. Efficacy and safety of atomoxetine for attention-deficit/hyperactivity disorder in children and adolescents-meta-analysis and meta-regression analysis. Psychopharmacology 2007;194(2):197-209.

Coleman 2009

Coleman JM, Abbott RA, Maughan B, Kuh D, Croudace TJ, Jones PB. Outcomes of conduct problems in adolescence: 40 year follow-up of national cohort. BMJ 2009;338(a2981):epub.

\section{Conners 1970}

Conners C. Symptom patterns in hyperkinetic, neurotic and normal children. Child Development 1970;41(3):66782.

\section{Conners 1998a}

Conners CK, Sitarenios G, Parker JDA, Epstein JN. The revised Conners' Parent Rating Scale (CPRS-R): factor structure, reliability, and criterion validity. Journal of Abnormal Child Psychology 1998;26(4):257-68.

\section{Conners 1998b}

Conners CK, Sitarenios G, Parker JD, Epstein JN. Revision and restandardization of the Conners' Teacher Rating Scale (CTRS-R): factor structure reliability, and criterion validity. Journal of Abnormal Child Psychology 1997;26(4):279-91.

\section{Conners 2001}

Conners CK. Conners' Rating Scales-Revised (Technical Manual). New York / Toronto: Multi-health Systems, 2001.

\section{Deeks 2005}

Deeks J, Macaskill P, Irwig L. The performance of tests of publication bias and other sample size effects in systematic reviews of diagnostic test accuracy was assessed. Journal of Clinical Epidemiology 2005;58(9):882-93.

\section{Deeks 2008}

Deeks JJ, Higgins JPT, Altman DG (editors). Chapter 9.5.2: Analysing data and undertaking meta-analyses. In: Higgins JPT, Green S editor(s). Cochrane Handbook for Systematic Reviews of Interventions. Chichester (UK): John Wiley \& Sons, 2008.

\section{Derogatis 1994}

Derogatis LR. Symptom Checklist-90-R: Administration, scoring, and procedures manual (3rd). Minneapolis, $\mathrm{MN}$ : National Computer Systems 1994.

\section{Dishion 2003}

Dishion TJ, Kavanagh K. Intervening in Adolescent Problem Behavior: A Family-Centered Approach. New York, NY: Guilford, 2003.

\section{Donner 2001}

Donner A, Piaggio G, Villar J. Statistical methods for the meta-analysis of cluster randomization trials. Statistical Methods in Medical Research 2001;10(5):325-38.

\section{Egger 1997}

Egger M, Davey-Smith G, Schneider M, Minder C. Bias in meta-analysis detected by a simple, graphic test. BMJ 1997; 315(7):629-34

\section{Enders 2001}

Enders CK. The performance of the full information likelihood estimator in multiple regression models with missing data. Educational and Psychological Measurement 2001;61(5):713-40.

Eyberg 1999

Eyberg S, Pincus D. Eyberg Child Behavior Inventory \& Sutter-Eyberg Student Behavior Inventory. Revised. Odessa, FL: Psychological Assessment Resources, 1999.

\section{Faraone 2003}

Faraone S, Sergeant J, Gillberg C, Biederman J. The worldwide prevalence of ADHD:is it an American condition? . World Psychiatry (www.pubmedcentral.nih.gov/ picrender.fcgi?artid=1525089\&blobtype=pdf) 2003; Vol. 2, issue 2:104-113 (accessed 13 March 2009).

\section{Faraone 2005}

Faraone SV, Perlis RH, Doyle AE, Smoller JW, Goralnick JJ, Holmgren MA, et al. Molecular genetics of attentiondeficit/hyperactivity disorder. Biological Psychiatry 2005;57 (11):1313-23.

\section{Farrington 1995}

Farrington DP. The development of offending and antisocial behaviour from childhood: key findings from the Cambridge study in delinquent development. Journal of Child Psychology and Psychiatry and Allied Disciplines 1995; 36(6):929-64.

Ford 2003

Ford T, Goodman R, Meltzer H. The British Child and Adolescent Mental Health Survey 1999: the prevalence of DSM-IV disorders. Journal of the American Academy of Child and Adolescent Psychiatry 2003;42:1203-11.

\section{Frankel 2003}

Frankel F. Measuring the quality of play dates. Unpublished MS, cited within Mikami 20102003.

\section{Furlong 2010}

Furlong M, McGilloway S, Bywater T, Hutchings J, Donnelly M, Smith SM, et al. Behavioural/cognitivebehavioural group-based parenting interventions for children age 3-12 with early onset conduct problems. Cochrane Database of Systematic Reviews 2010, Issue 1. DOI: $10.1002 / 14651858 . C D 008225$

\section{Gadow 1994}

Gadow KD, Sprafkin J. Child Symptom Inventories Manual. Stony Brook, NY: Checkmate Plus, 1994.

\section{Gresham 1990}

Gresham FM, Elliott SN. Social skills rating system. Social Skills Rating System. Circle Pines, MN: Assistance Service, 1990.

\section{Hardy 2007}

Hardy KK, Kollins SH, Murray DW, Riddle MA, Greenhill L, Cunningham C, et al. Factor structure of parentand Teacher-rated attention-deficit/hyperactivity disorder symptoms in the preschoolers with attention-deficit/ hyperactivity disorder treatment study (PATS). Journal of Child and Adolescent Psychopharmacology 2007;17(5): 621-33. 
Hartman 2003

Hartman R, Stage S, Webster-Stratton C. A growth curve analysis of parent training outcomes: examining the influence of child risk factors (inattention, impulsivity, and hyperactivity problems), parental and family risk factors. Journal of Child Psychology and Psychiatry and Allied Disciplines 2003;44(3):388-98.

\section{Higgins 2002}

Higgins JPT, Thompson SG. Quantifying heterogeneity in a meta-analysis. Statistics in Medicine 2002;21:1539-58.

\section{Higgins 2003}

Higgins J, Thompson SG, Deeks J, Altman D. Measuring inconsistency in meta-analyses. BMJ Clinical Research 2003; 327(7414):557-60.

\section{Higgins 2008a}

Higgins JPT, Green S, editors. Cochrane Handbook for Systematic Reviews of Interventions. Chichester (UK): John Wiley \& Sons, 2008.

\section{Higgins 2008b}

Higgins JPT, Altman DG (editors). Chapter 8: Assessing risk of bias in included studies. In: Higgins JPT, Green S editor(s). Cochrane Handbook for Systematic Reviews of Interventions. Chichester (UK): John Wiley \& Sons, 2008: $187-241$.

\section{Higgins 2008c}

Higgins JPT, Deeks JJ (editors). Chapter 7: Selecting studies and collecting data (specifically, section 7.7.3.8: Combining groups). In: Higgins JPT, Green S editor(s). Cochrane Handbook for Systematic Reviews of Interventions. Chichester (UK): John Wiley \& Sons, 2008:177.

\section{Hill 2008}

Hill C, Maskowitz K, Danis B, Wakschlag L. Validation of a clinically sensitive, observational coding system for parenting behaviors: The Parenting Clinical Observation Schedule. Parenting: Science and Practice 2008;8(2): 153-85.

Hutchings 2007

Hutchings J, Bywater T, Daley D, Gardner F, Whitaker C, Jones K, et al. Parenting intervention in Sure Start services for children at risk of developing conduct disorder: pragmatic randomised controlled trial. BMJ 2007;334 (678):7 pages.

\section{Jadad 1999a}

Jadad AR, Boyle MH, Cunningham C, Kim M, Scachar R. Treatment of Attention-Deficit/Hyperactivity Disorder. Evidence Report/Technology Assessment No. 11 (Prepared by McMaster University under Contract No. 290-97-0017). AHRQ Publication No. O0-E005. Rockville, Maryland: Agency for Healthcare Research and Quality, 1999. Available online at: www.ncbi.nlm.nih.gov/books/bv.fcgi? rid=hstat1.chapter.14677 (accessed August 2010).

\section{Jadad 1999b}

Jadad AR, Brooker L, Gauld M, Kakuma R, Boyle M, Cunningham CE, et al. The treatment of attention-deficit/ hyperactivity disorder: an annotated bibliography and critical appraisal of published systematic reviews and metaanalyses. Canadian Journal of Psychiatry 1999;44(10): 1025-35.

\section{Jensen 2001}

Jensen PS, Hinshaw SP, Kraemer HC, Lenora N, Newcorn $\mathrm{JH}$, Abikoff $\mathrm{HB}$, et al. ADHD comorbidity findings from the MTA study: comparing comorbid subgroups. Journal of the American Academy of Child and Adolescent Psychiatry 2001;40(2):147-58.

\section{Jensen 2007}

Jensen PS, Arnold LE, Swanson JM, Vitiello B, Abikoff $\mathrm{HB}$, Greenhill LL, et al. 3-year follow-up of the NIMH MTA study. Journal of the American Academy of Child and Adolescent Psychiatry 2007;46(8):989-1002.

\section{Johnston 1989}

Johnston C, Mash EJ. A measure of parenting satisfaction and efficacy. Journal of Clinical Child Psychology 1989;18 (2):167-75.

\section{Jones 2010}

Jones $S$. The art of medicine: bad seeds, bad science, and fairly black cats?. The Lancet 2010;376(9750):1384-5. DOI: 10.1016/S0140-6736(10)61944-7

\section{Kaufman 1997}

Kaufman J, Birmaher B, Brent D, Rao U, Flynn C, Moreci P, et al. Schedule for affective disorders and schizophrenia for school-age children - present and lifetime version (KSADS-PL): initial reliability and validity data. Journal of the American Academy of Child and Adolescent Psychiatry 1997; 37(7):695-702.

\section{Kazdin 1997}

Kazdin A. Parent management training: evidence, outcomes, and issues. Journal of the American Academy of Child and Adolescent Psychiatry 1997;36(10):1349-56.

\section{Klassen 2004}

Klassen AF, Miller A, Fine S. Health-related quality of life in children and adolescents who have a diagnosis of attention-deficit/hyperactivity disorder. Pediatrics 2004;114 (5):e541-e547.

\section{Kollins 2006}

Kollins S, Greenhill L, Swanson J, Wigal S, Abikoff H, McCracken J, et al. Rationale, design and methods of the preschool ADHD treatment study (PATS). Journal of the American Academy of Child and Adolescent Psychiatry 2006; 45(11):1275-83.

\section{Lahey 2004}

Lahey B, Pelham W, Loney J, Kipp H, Ehrhardt A, Lee SS, et al. Three-year predictive validity of DSM-IV attention deficit hyperactivity disorder in children diagnosed at 4-6 years of age. American Journal of Psychiatry 2004;161(11): 2014-20.

\section{Lefebvre 2008}

Lefebvre C, Manheimer E, Glanville J. Chapter 6: Searching for studies. In: Higgins JPT, Green S editor(s). Cochrane Handbook for Systematic Reviews for Interventions. Wiley, 2008. 


\section{Li 2006}

Li D, Sham PC, Owen MJ, He L. Meta-analysis shows significant association between dopamine system genes and attention deficit hyperactivity disorder. Human Molecular Genetics 2006;15(14):2276-84.

Lipsey 2001

Lipsey MW, Wilson DB. Practical Meta-Analysis. Sage, CA: Thousand Oaks, 2001.

\section{Mannuzza 1997}

Mannuzza S, Klein RG, Bessler A, Malloy P, Hynes ME. Educational and occupational outcome of hyperactive boys grown up. Journal of the American Academy of Child and Adolescent Psychiatry 1997;36(9):1222-27.

\section{Mannuzza 1998}

Mannuzza S, Klein RG, Bessler A, Malloy P, LaPadula M. Adult psychiatric status of hyperactive boys grown up. American Journal of Psychiatry 1998;155:493-98.

\section{McCarney 1995}

McCarney SB. The Attention Deficit Disorders Intervention Manual. Columbia, MO: Hawthorne Educational Services, Inc, 1995.

\section{McFadden 2010}

McFadden J. ADHD's roots are complex. To claim attention-deficit hyperactivity disorder is genetic is to dangerously simplify the nature of the condition. The Guardian (accessed online: www.guardian.co.uk/ commentisfree/2010/sep/30/attention-deficit-disordergenetic-roots/print) 30 September 2010.

\section{Michelson 2001}

Michelson D, Faries D, Wernicke J, Kelsey D, Kendrick K, Sallee F, et al. Atomoxetine in the treatment of children and adolescents with attention-deficit/hyperactivity disorder: a randomized, placebo-controlled, dose-response study. Pediatrics 2001;108(5):E83.

\section{Miller 1998}

Miller A, Lee S, Raina P, Klassen A, Zupancic J, Olsen L. A review of therapies for attention-deficit/hyperactivity disorder. Ottawa: Canadian Coordinating Office for Health Technology Assessment (CCOHTA) December 1998, issue Issue 2.

\section{Modesto-Lowe 2008}

Modesto-Lowe V, Danforth JS, Brooks D. ADHD: does parenting style matter?. Clinical Pediatrics 2008;47(9): $865-72$.

\section{Moher 2009}

Moher D, Liberati A, Tetzlaff J, Altman DG, The PRISMA Group. Preferred reporting items for systematic reviews and meta-analyses: The PRISMA statement. PLoS Medicine 2009;6:7. DOI: 10.1371/journal.pmed. 1000097

\section{MTA 2004}

MTA Cooperative Group. National Institute of Mental Health multimodal treatment study of ADHD followup: Changes in effectiveness and growth after the end of treatment. Pediatrics 2004;113(4):762-9.

\section{NICE 2000}

Lord J, Paisley S. The clinical effectiveness and cost-effectiveness of methylphenidate for hyperactivity in childhood. Technical Appraisal 13. London: National Institute for Clinical Excellence (NICE), August 2000.

\section{NICE 2006a}

National Institute for Health and Clinical Excellence \& Social Care Institute for Excellence. Parent-training/ education programmes in the management of children with conduct disorders. NICE Technology Appraisal 102 July 2006.

\section{NICE 2006b}

National Institute for Health and Clinical Excellence. Methylphenidate, atomoxetine and dexamfetamine for the treatment of attention deficit hyperactivity disorder in children and adolescents. Available online at: www.nice.org.uk/TA98 (accessed 14 February 2009).

\section{NICE 2008}

National Institute for Health and Clinical Excellence. Attention Deficit Hyperactivity Disorder: Diagnosis and management of ADHD in children, young people and adults. www.nice.org.uk/nicemedia/pdf/ ADHDConsFullGuideline.pdf January 2008:1-591 (accessed 9 September 2008).

\section{Oxman 1992}

Oxman A, Guyatt G. A consumer's guide to subgroup analyses. Annals of Internal Medicine 1992;116(1):78-84.

\section{Pelham 1998}

Pelham WE Jr, Wheeler T, Chronis A. Empirically supported psychosocial treatments for attention deficit hyperactivity disorder. Journal of Clinical Child Psychology 1998;27(2):190-205.

\section{Pelham 2008}

Pelham WE Jr, Fabiano GA. Evidence-based psychosocial treatments for attention-deficit/hyperactivity disorder. Journal of Clinical and Child Adolescent Psychology 2008;37 (1):184-214.

\section{Pliszka 2007}

Pliszka S, Bernet W, Bukstein O, Walter H, Arnold V, Beitchman J, et al. Practice Parameters for the Assessment and Treatment of Children and Adolescents with Attention Deficit Hyperactivity Disorder. Journal of the American Academy of Child and Adolescent Psychiatry 2007;46(7): 894-921.

\section{Rasmussen 2000}

Rasmussen P, Gillberg C. Natural outcome of ADHD with developmental coordination disorder at age 22 years: A controlled, longitudinal, community-based study. Journal of the American Academy of Child and Adolescent Psychiatry 2000;39(11):1424-31.

\section{RevMan 2008 [Computer program]}

The Nordic Cochrane Center, The Cochrane Collaboration. Review Manager (RevMan). Version 5.0. Copenhagen: The Nordic Cochrane Center, The Cochrane Collaboration, 2008 . 


\section{Reynolds 1998}

Reynolds CR, Kamphaus RW. Behaviour Assessment System for Children. Circle Pines, MN: American Guidance Service, 1998

\section{Rostain 1993}

Rostain AL, Power TJ, Atkins MS. Assessing parents' willingness to pursue treatment for children with attentiondeficit hyperactivity disorder. Journal of the American Academy of Child Adolescent Psychiatry 1993;32(1):175-81.

Santosh 2005

Santosh PJ, Taylor E, Swanson J, Wigal T, Chuang S, Davies $\mathrm{M}$, et al. Refining the diagnoses of inattention and overactivity syndromes: A reanalysis of the Multimodal Treatment study of attention deficit hyperactivity disorder (ADHD) based on ICD-10 criteria for hyperkinetic disorder. Clinical Neuroscience Research 2005;5:295-306.

\section{Scahill 2008}

Scahill L. Email to Jane Dennis confirming no separate analysis had been undertaken for ADHD subset (and questionning why it should, given small sample size) 2008 (12 September).

\section{Schünemann 2008}

Schünemann HJ, Oxman AD, Higgins SPT, Vist GE, Glasziou P, Guyatt GH. Chapter 11: Presenting results and 'Summary of findings' tables. In: Higgins JPT, Green S editor(s). Cochrane Handbook for Systematic Reviews of Interventions. Chichester (UK): John Wiley \& Sons, 2008: 335-59.

\section{Sciutto 2007}

Scuitto M, Eisenberg M. Evaluating the evidence for and against the overdiagnosis of ADHD. Journal of Attention Disorders 2007;11(2):106-13.

\section{Scott 2001b}

Scott S, Knapp M, Henderson J, Maughan M. Financial cost of social exclusion: follow up study of antisocial children into adulthood. BMJ 2001;323:1-5. DOI: 10.1136/bmj.323.7306.191

Scott 2007

Scott S. Conduct disorders in children. BMJ 2007;334: 646. DOI: 10.1136/bmj.39161.370498.BE

\section{Scott 2011}

Scott S. Personal communication (by email and telephone, with Morris Zwi) January 2011.

\section{SIGN 2009}

Scottish Intercollegiate Guidelines Network. Management of attention deficit and hyperkinetic disorders in children and young people: A national clinical guideline. Available from: http://www.sign.ac.uk/pdf/sign112.pdf October 2009 (accessed 2 November 2011).

\section{Solanto 2001}

Solanto M, Abikoff H, Sonuga-Barke E, Schachar R, Logan GD, Wigal T, et al. The ecological validity of delay aversion and response inhibition as measures of impulsivity in ADHD: a supplement to the NIMH multi-modal treatment study of AD/HD. Journal of Abnormal Child Psychology 2001;29:215-28.

\section{Sonuga-Barke 2003a}

Sonuga-Barke E, Daley D, Thompson M, Swanson J. Preschool ADHD: Exploring uncertainties in diagnostic validity and utility, and treatment efficacy and safety. Expert Review Neurotherapeutics 2003;3(4):465-76.

\section{Sonuga-Barke 2003b}

Sonuga-Barke E, Dalen L, Remington B. Do executive deficits and delay aversion make independent contributions to preschool Attention-Deficit/Hyperactivity Disorder symptoms?. Journal of the American Academy of Child \& Adolescent Psychiatrists 2003;42(11):1335-42.

Sourander 2005

Sourander A, Helstelä L. Childhood predictors of externalizing and internalizing problems in adolescence: a prospective follow-up study from age 8 to16. European Child and Adolescent Psychiatry 2005;14(8):415-23.

Sterne 2001

Sterne J, Egger M. Funnel plots for detecting bias in metaanalysis: guidelines on choice of axis. Journal of Clinical Epidemiology 2001;54(10):1046-55.

\section{Sterne 2008}

Sterne JAC, Egger M, Moher D (editors). Chapter 10.4: Detecting reporting biases. In: Higgins JPT, Green $S$ editor(s). Cochrane Handbook for Systematic Reviews of Interventions. Chichester (UK): John Wiley \& Sons, 2008: 310-324.

Swanson 1983

Swanson JM, Sandman CA, Deutsch C, Baren M. Methylphenidate hydrochloride given with or before breakfast: !. Behavioral, cognitive and electrophysiological effects. Pediatrics 1983;72:49-55.

Taylor 1996

Taylor E, Chadwick O, Heptinstall E, Danckaerts M. Hyperactivity and conduct problems as risk factors for adolescent development. Journal of the American Academy of Child and Adolescent Psychiatry 1996;35(9):1213-26.

\section{Taylor 2004}

Taylor E, Döpfner M, Sergeant J, Asherson P, Banaschewski T, Buitelaar J, et al. European clinical guidelines for hyperkinetic disorder - first upgrade. European Child and Adolescent Psychiatry 2004;13 Suppl 1:7-30.

\section{Webster-Stratton 1998}

Webster-Stratton C, Hancock L. Parent training for young children with conduct problems. Content, methods and therapeutic process. In: Schaefer CE editor(s). Handbook of Parent Training. New York: Wiley, 1998.

\section{Weiss 1985}

Weiss G, Hechtman L, Milroy T, Perlman T. Psychiatric status of hyperactives as adults: A controlled prospective 15-year follow-up of 63 hyperactive children. Journal of the American Academy of Child and Adolescent Psychiatry 1985; 24(2):211-20. 


\section{Wells 2000}

Wells K, Pelham W, Kotkin R, Hoza B, Abikoff HB, Abramowitz A, et al. Psychosocial treatment strategies in the MTA study: rationale, methods, and critical issues in design and implementation. Journal of Abnormal Child Psychology 2000;28(6):483-505.

\section{Whitehead 1994}

Whitehead A, Jones NM. A meta-analysis of clinical trials involving different classifications of response into ordered categories. Statistics in Medicine 1994;13(23-24):2503-15. MEDLINE: 95215666

\section{WHO 1977}

World Health Organization. International Statistical Classification of Diseases and Related Health Problems, 9th Revision (ICD-9). Geneva: World Health Organization, 1977.

\section{WHO 1992}

World Health Organization. International Statistical Classification of Diseases and Related Health Problems. 10th Edition. Geneva: World Health Organization, 1992.

\section{Williams 2010}

Williams NM, Zaharieva I, Martin A, Langley K, Mantripragada K, Fossdal R, et al. Rare chromosomal deletions and duplications inattention-deficit hyperactivity disorder: a genome-wide analysis. The Lancet 2010;376 (9750):1401-8. DOI: 10.1016/S0140-6736(10)61109-9

\section{Yusuf 1991}

Yusuf S, Wittes J, Probstfield J, Tyroler HA. Analysis and interpretation of treatment effects in subgroups of patients in randomized clinical trials. JAMA 1991;266(1):93-8.

* Indicates the major publication for the study 


\title{
CHARACTERISTICS OF STUDIES
}

\section{Characteristics of included studies [ordered by year of study]}

\author{
Blakemore 1993
}

Methods

Design: Randomised controlled trial.

Participants

Participants: The participating family had to have at least one child aged 6-11 who satisfied the criteria for ADHD according to DSM III-R criteria. Some children taking methylphenidate (investigators sought to balance numbers between groups).

Age: 6-11 years (no mean given).

Gender: Not mentioned.

Number: 24 (8 in each treatment arm).

Setting: The Learning Center, Calgary, Canada, for children and adults with learning difficulties.

Inclusion criteria: At least one child aged 6-11 who satisfied DSM III-R criteria for ADHD. The children had to show evidence of ADHD in a wide range of situations and the problems must have been evident before the age of six.

Exclusion criteria: Evidence of a serious neurological difficulty in the child, evidence of a serious marital difficulty or where the child met criteria for CD

Interventions

Group treatment: 12 weekly two hour sessions in which eight families meet with two therapists. Two follow-up sessions offered at three and six months after the last session, the topics of which are suggested by the parents.

Session 1: Perspective shift - introduces the project to the parents.

Session 2: Selecting behaviours for intervention.

Session 3: Behaviour management procedures.

Session 4: Refining the behaviour management script.

Session 5: The grief cycle.

Session 6: Communicating skills.

Session 7: Listening.

Session 8: Acknowledging feelings.

Session 9: Self-esteem.

Session 10: Anger management 1.

Session 11: Anger management 2.

Session 12: Review.

Individual treatment: Identical to group programme but families meet with just one therapist on their own and sessions lasted for one hour not two.

Waiting list control: Offered a group treatment after all control data had been collected

Primary outcomes:

Changes in the child's general behaviour: Eyberg Child Behaviour Inventory (Achenbach 1991).

\section{Secondary outcomes:}

Parental Stress: Parenting Stress Index (PSI) (Abidin 1986). Mothers' results compared with fathers'.

Outcome measures unable to use: 


$\begin{array}{ll}\text { Notes } & \text { No figures given for outcomes. Only small, poor quality graphs shown for (1) Parenting } \\ \text { Stress Index (2) Frequency of problem behaviours for mothers and fathers as function } \\ \text { of treatment conditions and (3) Mother's problem solving performance. } \\ \text { Consultation on issues related to the project was offered to teachers of participating chil- } \\ \text { dren. This included "group presentations of the project material" or individual sessions. } \\ \text { Topics included behaviour management, mediational communication, ADHD overview } \\ \text { amongst others }\end{array}$

\section{Risk of bias}

\begin{tabular}{|c|c|c|}
\hline Bias & Authors' judgement & Support for judgement \\
\hline $\begin{array}{l}\text { Random sequence generation (selection } \\
\text { bias) }\end{array}$ & Unclear risk & $\begin{array}{l}\text { Quote: "randomly assigned to one of three treat- } \\
\text { ment conditions: group treatment, individual } \\
\text { treatment or waiting list control...A stratified } \\
\text { sampling procedure is used so that the groups } \\
\text { are balanced with respect to age of child, num- } \\
\text { ber of children who meet the criteria for ODD } \\
\text { and the number of children who are taking Ri- } \\
\text { talin" (p71). } \\
\text { Comment: Precise method not stated. Authors } \\
\text { were contacted for clarification but no clarifica- } \\
\text { tion received }\end{array}$ \\
\hline
\end{tabular}
ification but no clarification received

Blinding (performance bias and detection High risk bias) of participants?

Blinding (performance bias and detection High risk bias)

of those delivering intervention?

Blinding (performance bias and detection Low risk bias)

of outcome assessors?

Incomplete outcome data (attrition bias) High risk All outcomes

Selective reporting (reporting bias)

High risk
Cannot be blinded to the parent training intervention.

Cannot be blinded as they are delivering the parent training intervention
For the follow-up evaluation session the "research assistant interviewing the mothers was blinded to the treatment status of the parent" (p. 80)

This paper presented preliminary findings, not all data had been analysed so not all outcomes had been addressed

Protocol for initial study not available. Not all data had been analysed at the time of publication. it is unclear why measures used at baseline 

not used for programme evaluation. Furthermore, when outcomes of interest to the review are reported they are only done in graph format which are difficult to interpret, with only means and no standard deviations and no numerical data given in the text. Investigators also not that they did not evaluate fathers' data for Group treatment due to "large pre-test differences...." bias due to paper being a preliminary report. Data are presented only in graph form without exact figures, therefore it is difficult to interpret accurately remainder 'had an average of six symptoms inattentive symptoms and seven hyperactiveimpulsive symptoms", for “at least six months" (p.13). ...'Majority '(p.15) of children 'were taking psychoactive medication for behavioral problems throughout the study'. Investigators interested in maternal stress and note at least half the mothers scored a standard deviation above the mean for non-patient norms on the General Severity Index of the Symptoms Checklist 90-Revised (SCL-90-R, Derogatis 1994).

Age: $5-9$ years (means across groups $=1=6.94(\mathrm{SD}=1) ; 2=6.56(\mathrm{SD}=1.03) ; 6.88$ $(\mathrm{SD}=1.36))$.

Gender: 38 boys, 10 girls.

Number: 54 in initial sample, 48 completed (16 in each group).

Setting: Psychological Services Centre, University of Memphis, Memphis, Tennessee, USA.

Inclusion criteria: Child aged between five and nine, child was planning to participate in structured setting outside the home, child not diagnosed with "mental retardation" or pervasive developmental disorder, child must have been diagnosed with ADHD using DSM-IV criteria.

Exclusion criteria: "Mental retardation" and pervasive developmental disorder.

Parent training group: 8 week manualised course focusing on teaching parents specialised child management techniques primarily involving contingency management (orientation, principles of behaviour management, parental attending to child behaviour, home token system, response cost, time out from reinforcement and child management in public areas).

Parent training and self management group: 8 weeks of the parent training intervention and components of a cognitive behavioural intervention designed to develop emotional management skills (measuring mood, effective listening, identifying and modifying cognitive distortions, cost benefit analysis, externalising voices, identifying and modifying 
silent assumptions and building self-esteem).

Control group: Mothers were kept on a waiting list for 8 weeks then were given the option to receive the combined intervention

Primary outcomes:
Changes in the child's ADHD-related behaviour
ADDES-Home (Hyperactive Impulsive Scale) (McCarney 1995)
ADDES-Home (Inatttentive Scale) (McCarney 1995)
ADDES-School (McCarney 1995) (Total scale; Inattentive and Hyper-Impulsive scales
Change in the child's ADHD-symptom-related behaviour in school setting
Teacher ratings of child behavior of the school versions of instruments listed above
Changes in general child behaviour
The CBCL Total Problems Scale (Achenbach 1986) (measured by both parent and
teacher)
The CBCL Externalizing Scale (Achenbach 1986) (measured by both parent and teacher)
The CBCL Internalizing Scale (Achenbach 1986) (measured by both parent and teacher)
Secondary outcome:
Parenting stress: The Revised Symptom Checklist (SCL-90-R) (Derogatis 1994).

Notes

Risk of bias

\begin{tabular}{|c|c|c|}
\hline Bias & Authors' judgement & Support for judgement \\
\hline $\begin{array}{l}\text { Random sequence generation (selection } \\
\text { bias) }\end{array}$ & Unclear risk & $\begin{array}{l}\text { Quote "mothers were ranked according to their } \\
\text { GSI score on the SCL-90-R...were then sepa- } \\
\text { rated into three groups based on their score and } \\
\text { availability...each group was then randomly as- } \\
\text { signed parent training, parent training plus self } \\
\text { management or waiting-list control" (p. 23). } \\
\text { Comment: Precise method not stated; investi- } \\
\text { gator could not be contacted }\end{array}$ \\
\hline
\end{tabular}

Allocation concealment (selection bias) Unclear risk

Quote "mothers were ranked according to their GSI score on the SCL-90-R...were then separated into three groups based on their score and availability...each group was then randomly assigned parent training, parent training and SM or waiting-list control” (p. 23).

Comment: Precise method not stated; investigator could not be contacted

Blinding (performance bias and detection High risk bias)

Cannot be blinded to the parent training interof participants? vention. 
Blinding (performance bias and detection High risk bias)

of those delivering intervention?

Blinding (performance bias and detection High risk bias)

of outcome assessors?

Incomplete outcome data (attrition bias) Low risk All outcomes
Cannot be blinded as they are delivering the parent training intervention

Research assistants analysing data were not blind to subject treatment conditions as they also provided child care if parents brought their children to sessions

54 mother-child pairs originally, 6 were excluded because one parent did not complete the baseline assessment or attend any treatment sessions, five parents dropped out of the study and were unable or unwilling to complete post-treatment assessments. Investigators did compare dropouts to those who attended and reported no significant differences between these groups and conducted separate MANOVA analyses including first only those who attended a majority of sessions and secondly, all participants regardless of attendance (which accounts for 55 of the initial 56 pairs)

Selective reporting (reporting bias) High risk

Protocol for study unavailable. Repeated attempts to contact the author for this data were unsuccessful. Tables within dissertation do not report all means and standard deviations for all outcomes for all groups (for example, Table 19 does so only for three outcomes which are significant for one or both intervention groups). No data from teacher reports are mentioned at all beyond that 'findings were not significant' (pp 37-8)

Other bias

Unclear risk

Insufficient information to assess risk of other bias.

Lehner-Dua 2001

Methods

Participants
Design: Randomised controlled trial

Participants: 48 parents from families with children aged 6-11 years, recently diagnosed with ADHD by a mental health professional according to DSM-IV criteria.

Age: 6-10 years (median age $=8$ ).

Gender: 33 boys, 15 girls.

Number: 48.

Setting: Hofstra University's Centre for Psychological Evaluation, Research and Counselling Clinic, New York, USA. 
Inclusion criteria: Children aged 6-11 years, recently diagnosed with ADHD by a mental health professional according to DSM-IV criteria

Interventions

Parent Training Groups: Based on the "Defiant Children" programme developed by Barkley (Barkley 1997). 10 × 2 hour structured sessions of parent training on a weekly basis for 9 weeks with a booster session 1 month after the 9th session. At each session new concepts and skills were introduced, parent handouts were reviewed, new parent behaviours were modelled, parents rehearsed new skills and the homework assignment was reviewed.

week 1: why children misbehave,

week 2: pay attention,

week 3: increasing compliance and independent play,

week 4: when praise is not enough, poker chips and points,

week 5: time out! and other disciplinary methods,

week 6: extending time out to other misbehaviors,

week 7: anticipating problems, managing children in public places,

week 8: improving school performance from home, the daily school behaviour report card,

week 9: handling future behaviour problems,

week 10: booster session and follow up meetings.

Control Groups: Parental discussion support group. This group met for 9 weekly 2 hour sessions and one follow-up session 1 month later. There was no structured programme, parents discussed any problems raised by group members, there were no handouts and no homework assignments. After data collection parents were debriefed, given a summary of the results and any questions were answered

\section{Outcomes}

Primary outcomes:

Parents perceived severity of child's ADHD symptoms:

Behaviour Assessment System for Children (BASC) (Reynolds 1998).

\section{Secondary outcomes:}

Change in parenting skills

Parenting Sense of Competence (PSOC) (Johnston 1989).

Parental level of stress

Parent Stress Index (PSI) (Abidin 1986) (both as subscales, Child and Parent Domains)

Outcomes not used in this review

Total scores on the PSI were not used in this review as they were simple totals of the CD and PD domains of the PSI used elsewhere in meta-analysis; Life Stress is not used

Notes

Risk of bias

\section{Bias}

Random sequence generation (selection Unclear risk bias)
Authors' judgement

\section{Support for judgement}

Quote: “In order to divide the participants into two groups, each participant chose a group time that was convenient for them. The groups were then randomly assigned a letter either A or B. The participants in A were the experimental 

ticipants in B were the control group (parent support). There were two groups of each in order to keep the group sizes to a workable number and provide greater availability for participants" (p 42)

\begin{tabular}{l|l|l}
\hline Allocation concealment (selection bias) & Unclear risk & Precise method not stated. \\
\hline $\begin{array}{l}\text { Blinding (performance bias and detection } \\
\text { bias) } \\
\text { of participants? }\end{array}$ & High risk & Participants cannot be blinded to intervention. \\
\hline $\begin{array}{l}\text { Blinding (performance bias and detection } \\
\text { bias) } \\
\text { of those delivering intervention? }\end{array}$ & High risk & $\begin{array}{l}\text { Those delivering intervention cannot be blinded } \\
\text { - least of all in these conditions: "the same exper- } \\
\text { imenter ran both the parent training and sup- } \\
\text { port groups." (p 83) }\end{array}$ \\
\hline
\end{tabular}

Blinding (performance bias and detection High risk bias)

of outcome assessors?
High risk All outcomes
Blinding of outcome assessors not mentioned and highly unlikely - it would seem the investigator conducted her own assessments as well as having run both the structured treatment and the parent support group

Initially 80 calls from parents, 68 accepted to begin programme, 61 parents began the study, 48 parents completed the programme. (Barkley group began with 27 participants, 23 completed; Support group started with 34 participants, 25 completed). Drop out reasons given as shifting work schedules, family crisis and non-applicability of groups. Dropouts uneven between groups

Selective reporting (reporting bias) Unclear risk

High risk
No obvious selective reporting but in the absence of a study protocol we cannot be clear

Investigator criticised her own design as follows: "A no contact group may be integrated in future research to be sure that the results were not due to just attention and/or contact with the participants - statistically significant improvements in both the parent training and support groups for parents' sense of competence" (p 83)

"The same experimenter ran both the parent training and support groups." (p 83) Large risk of contamination

"One parent in the support group had a friend in the parent training group which whom she 
Lehner-Dua 2001 (Continued)

compared notes." (p83). Investigator considers adding a confidentiality clause in future experiments

van den Hoofdakker 2007

\begin{tabular}{ll}
\hline Methods & Design: Randomised controlled trial \\
\hline \multirow{2}{*}{ Participants } & Participants: Children who met DSM-IV criteria for ADHD (full scale IQ of the WISC- \\
& III-R for children under 6). \\
& Age: $4-12$ (mean age 7.4, SD $=1.9)$. \\
& Gender: 76 boys, 20 girls. \\
& Number: 96. \\
& Setting: Mental health outpatient clinics, the Netherlands. \\
& Inclusion criteria: Meet DSM-IV criteria for ADHD, IQ>80 (Full Scale IQ of the WISC- \\
& III-R, for children under the age of 6 years the Full Scale IQ of the QWPPSI-R), age \\
& between $4-12$ years, both parents (if present) willing to participate in the BPT program. \\
& Exclusion criteria \\
\hline
\end{tabular}

Interventions

Behavioural Parent Training + Routine Clinical Care: Manual based parent training consisted of $12 \times 120$ minute group sessions over 5 months for 6 children's parents at a time. Sessions led by two psychologists, specific target behaviours were established for each child. Most techniques were drawn from Barkley (1987) and Forehand \& McHahon (1981). Parenting skills addressed were: structuring the environment, setting rules, giving instructions, anticipating misbehaviors, communicating, reinforcing positive behaviour, ignoring, employing punishment, and implementing a token system. Psychoeducation and restructuring of parental cognitions were also important elements. Homework assignments were given and parents read chapters from a specially written book by van der Veen-Mulders (2001). Each week parents practiced the skills and wrote reports after the exercises. Follow up assessment 25 weeks post-intervention.

Routine Clinical Care: Carried out by four experienced senior child and adolescent psychiatrists. They provided care as usual including supportive counselling, psychoeducation, pharmacotherapy, and crisis management whenever necessary. Checkups were usually every 3-6 months. Parents were given the group parent training after all data had been collected

Primary outcomes:

Change in the child's ADHD-symptom-related behaviour in home setting: The ADHD Index subscale of the Conners' parent Rating Scale-Revised Short Form (CPRS-R-S) (Conners 2001).

Changes in the child's general behaviour: Externalizing and Internalizing subscales of the Dutch version of the Child Behaviour checklist (CBCL) (Achenbach 1991).

Secondary outcomes:

Parental Stress: Parenting Stress Index (Abidin, 1983).

Notes

Risk of bias

Parent training interventions for Attention Deficit Hyperactivity Disorder (ADHD) in children aged 5 to 18 years (Review)

Copyright @ 201 I The Cochrane Collaboration. Published by John Wiley \& Sons, Ltd. 
van den Hoofdakker 2007 (Continued)

\begin{tabular}{|c|c|c|}
\hline Bias & Authors' judgement & Support for judgement \\
\hline $\begin{array}{l}\text { Random sequence generation (selection } \\
\text { bias) }\end{array}$ & Unclear risk & $\begin{array}{l}\text { Quote: "randomized block design" (p.1265). No } \\
\text { method specified }\end{array}$ \\
\hline Allocation concealment (selection bias) & Unclear risk & Not described. \\
\hline $\begin{array}{l}\text { Blinding (performance bias and detection } \\
\text { bias) } \\
\text { of participants? }\end{array}$ & High risk & Participants cannot be blinded to intervention. \\
\hline $\begin{array}{l}\text { Blinding (performance bias and detection } \\
\text { bias) } \\
\text { of those delivering intervention? }\end{array}$ & High risk & $\begin{array}{l}\text { Those delivering intervention cannot be } \\
\text { blinded. }\end{array}$ \\
\hline $\begin{array}{l}\text { Blinding (performance bias and detection } \\
\text { bias) } \\
\text { of outcome assessors? }\end{array}$ & Unclear risk & Blinding of outcome assessors not mentioned. \\
\hline $\begin{array}{l}\text { Incomplete outcome data (attrition bias) } \\
\text { All outcomes }\end{array}$ & Low risk & $\begin{array}{l}\text { Investigators described using intention-to-treat } \\
\text { analysis for missing data (using a last-observa- } \\
\text { tion-carried-forward method) }\end{array}$ \\
\hline Selective reporting (reporting bias) & High risk & $\begin{array}{l}\text { All outcomes prospectively stated have been } \\
\text { reported. However, they collected information } \\
\text { from both parents separately but state that: "In } \\
\text { this study we analyzed the data from the moth- } \\
\text { ers" (p 1266) }\end{array}$ \\
\hline Other bias & Low risk & $\begin{array}{l}\text { Study appears to be free from other sources of } \\
\text { bias. }\end{array}$ \\
\hline
\end{tabular}

\section{Mikami 2010}

Methods

Participants
Design: Randomised controlled trial

Participants: Participants were families of 62 children ( 42 boys) with ADHD. A comparison group of 'normal' children (62 age- and sex-matched children) were also recruited. Diagnoses of those with ADHD were made via Child Symptom Inventory (CSI, Gadow 1994) and diagnoses were verified in a clinical review with parents using the K-SAD (Kaufman 1997). Majority of children with ADHD were DSM-IV Combined Type (ADHD-C; $\mathrm{n}=46$ ) and the remainder were DSM-IV Inattentive Type (ADHD-I; $\mathrm{n}=$ 16). Data on ethnicity provided for the whole sample. Most children (85\%) classed as white, 5\% African American, 2\% Asian American, 1\% Latino and 7\% of more than one race. Each child participated with one parent 'most involved in a child's social life', 94\% of whom were female. Children on medication $(n=40)$ for 3 months prior to study were permitted to continue on the same regime

Age: $6-10$ years $($ mean $=8.26, \mathrm{SD}=1.21)$. 
Gender: 42 boys, 20 girls.

Number: 62 (30 in intervention, 32 in control arm).

Setting: Recruitment was from clinics, schools, paediatricians and from a database of families who had previously participated in research at the University of Virginia (Charlottesville, Virginia, USA).

Inclusion criteria: Children with ADHD.

Exclusion criteria: Pervasive developmental disorders, full scale IQ below 70 or verbal IQ below 75. Anxiety/depressive disorders, ODD and CD were permitted, although no child met criteria for $\mathrm{CD}$. No child could be receiving other psychosocial treatment for social or behavioural issues; however, academic interventions were allowed

Interventions

Parental friendship coaching (PFC) was provided in eight 90 minute group sessions, delivered once weekly, involving 5 to 6 parents and led by two clinicians. The parent who had originally completed questionnaire and attended baseline playgroup assessment was requested to attend PFC, but other parent could attend if wished. Sessions were manualised. One month after the study ended, parents were contacted by phone and interviewed regarding changes in their child's peer relationships

Topic I: Setting a foundation for effective coaching by improving the parent-child relationship

Session 1: Building a positive parent-child relationship by using 'attending' and 'special time'; How antecedents and consequences shape behaviour

Session 2: Using active listening when discussing child's social concerns; providing effective praise and constructive feedback to your child

Topic II: Coaching your child in social skills needed for good peer relationships

Session 3: Teaching child good dyadic play skills

Session 4: Choosing the right peer to foster a friendship with your child; Meeting new friends through unstructured and organised activities

Topic III: Organising playdates that will foster the development of good friendships

Session 5: Inviting a peer for a playdate; how parents can network with other parents and set a good social example

Session 6: Preparing the playdate setting as the host to prevent boredom and conflict among the children

Session 7: Debriefing with your child after the playdate; Preparing your child for a playdate as a guest

Topic IV: Review and future directions

Session 8: Recap of skills taught; reasons for backsliding; what to expect in the future

Homework issued with each session involving worksheets, practice sessions, discussions with the child and setting up playdates. Group viewing of videotapes of parental interaction was used as a teaching tool

Control group: No treatment, but after follow-up, control group parents were offered a workshop summarising PFC content 


\begin{tabular}{|c|c|}
\hline & $\begin{array}{l}\text { Outcome measures unable to use: } \\
\text { Quality of Play Questionnaire (QPQ) (Frankel 2003) (questionnaire only available on } \\
\text { an unpublished manuscript) } \\
\text { Child friendships at follow-up (global } 5 \text { point questionnaire completed by parent) } \\
\text { Parental Behaviour in Playgroup (socialising, facilitation and corrective feedback) (video- } \\
\text { tapes coded by blinded observers on a scale of 10) } \\
\text { Parental Behaviour in Parent-child interaction (coded as above using a Likert scale from } \\
0 \text { to 3) } \\
\text { Playdates Hosted }\end{array}$ \\
\hline Notes & Funding from NIMH grant \\
\hline
\end{tabular}

\section{Risk of bias}

\begin{tabular}{l|l} 
Bias Authors' judgement & Support for judgement
\end{tabular}

Random sequence generation (selection Unclear risk bias)
Quote: "randomly assigned to receive PFC or to be in a no-treatment control group". Method of randomisation not described. It is clear that six cohorts were randomised in a stratified manner, each cohort containing five to six playgroups. Each playgroup contained one parent receiving $\mathrm{PFC}$, one parent receiving no treatment, and two other parents of children without ADHD, who received no intervention

Not described.

Participants cannot be blinded to intervention.

Blinding (performance bias and detection High risk bias)

of participants?

Blinding (performance bias and detection High risk bias)

of those delivering intervention?

Blinding (performance bias and detection Low risk bias)

of outcome assessors?
Those delivering intervention cannot be blinded.

Blinding of outcome assessors was mentioned for those assessing videotaped interactions. Blinding is not mentioned for other outcomes, but it seems likely that this was attended to given the rigour relating to the videotaped outcomes. Also, "although parents were obviously aware of whether or not they had received PFC, study personnel kept teachers unaware of the family's treatment status and asked parents to not give teachers this information" (page 740) (Enders 2001). 


\begin{tabular}{l|l|l}
\hline $\begin{array}{l}\text { Incomplete outcome data (attrition bias) } \\
\text { All outcomes }\end{array}$ & Low risk & $\begin{array}{l}\text { Investigators described using intention-to-treat } \\
\text { analysis for missing data (p. 744) using “full in- } \\
\text { formation maximum likelihood methods" }\end{array}$ \\
\hline Selective reporting (reporting bias) & Unclear risk & $\begin{array}{l}\text { All likely outcomes appear to be reported but } \\
\text { in the absence of the trial's protocol judgement } \\
\text { must remain 'unclear' }\end{array}$ \\
\hline Other bias & Low risk & $\begin{array}{l}\text { In one cohort, a parent of a child with ADHD } \\
\text { (chosen randomly) was assigned to treatment. } \\
\text { Steps werehowever taken to test no demographic } \\
\text { differences existed at baseline between the two } \\
\text { ADHD groups }\end{array}$ \\
\hline
\end{tabular}

\section{Characteristics of excluded studies [ordered by study ID]}

\begin{tabular}{|c|c|}
\hline Study & Reason for exclusion \\
\hline Abikoff 2004 & $\begin{array}{l}\text { RCT of children with ADHD. Three arms: methylphenidate plus psychosocial intervention versus } \\
\text { methylphenidate alone versus methylphenidate plus attentional control. Excluded because psychosocial } \\
\text { intervention involves social skills training involving direct intervention with the children }\end{array}$ \\
\hline Aman 2010 & RCT. Children diagnosed with pervasive developmental disorders, not ADHD \\
\hline Anastopoulos 1993 & $\begin{array}{l}\text { Appeared to be RCT or at least quasi-RCT of parent training versus wait list control for children diagnosed } \\
\text { with DSM-III-R ADHD, based on parents' responses to interview questions; in fact, study not even quasi- } \\
\text { randomised (this was confirmed by personal contact with investigator) - "subjects were in groups as a function } \\
\text { of when they requested services" (Anastopoulous 2009) }\end{array}$ \\
\hline Arnold 2007 & $\begin{array}{l}\text { Uncontrolled intervention study - participants were a convenience sample of four adolescents and families } \\
\text { (part of Masters' thesis) }\end{array}$ \\
\hline Baker-Ericzen 2010 & Review article (focused moreover on disruptive behaviour disorders rather than ADHD) \\
\hline Bandsma 1997 & $\begin{array}{l}\text { This three-armed, apparently nonrandomised intervention study involves 'group mediation therapy' with } \\
\text { three groups which appear to be clinically different from one another (those with clinically defined ADHD, } \\
\text { those with borderline ADHD symptoms, and a 'norm group'). Triallists state that the study was not designed } \\
\text { with a control group'. Furthermore, the nature of the intervention (mediation therapy) appears to involve } \\
\text { direct work with children }\end{array}$ \\
\hline Barkley 1992 & $\begin{array}{l}\text { RCT of youths aged } 12-18 \text { with ADHD. Three 'family' interventions were compared, none of which } \\
\text { met inclusion criteria (interventions consisted of behavior management training; problem-solving and } \\
\text { communication training; structural family therapy) }\end{array}$ \\
\hline
\end{tabular}

Parent training interventions for Attention Deficit Hyperactivity Disorder (ADHD) in children aged 5 to 18 years (Review) 


\begin{tabular}{l|l}
\hline Barkley 2000 & $\begin{array}{l}\text { RCT of children with 'disruptive behaviour'; participants were too young or of insecure diagnosis (screening } \\
\text { test involved parent report only) to be included within this review }\end{array}$ \\
\hline Barkley 2001 & $\begin{array}{l}\text { RCT of adolescents with ADHD. Both interventions were 'active' and involved family therapies, which } \\
\text { involved both parents and direct work with adolescents, using Behaviour Management Training and Prob- } \\
\text { lems Solving Communication Training }\end{array}$ \\
\hline Barkley 2002b & $\begin{array}{l}\text { RCT of preschool children lacking formal a formal diagnosis of ADHD at entry into the trial. They were } \\
\text { randomised to parent training, special kindergarten enrichment classroom only, the combined treatment } \\
\text { condition and a no treatment condition }\end{array}$ \\
\hline
\end{tabular}

Beyer 1994 RCT (conducted in course of a PhD). Age range problematic (3-11) (separate data not available for children over 5, according to the author); also formal diagnosis of ADHD lacking in some participants

Bogle 2007 RCT wherein children (only some of whom had a formal diagnosis of ADHD) were randomised to one of two active treatments, i.e., a 'Challenging Horizons Programme' plus 'Academic Skills Building Workshop' or 'Challenging Horizons Programme' only. This intervention does not meet inclusion criteria as direct interventions with the children were used and there is no no-treatment control group

Bor 2002 RCT wherein participants were aged between 36 and 48 months and had no formal diagnosis of ADHD. Participants were randomised to enhanced behavioural family intervention, standard family behavioural intervention or wait list control group

Chacko 2007 RCT of children with ADHD. Excluded because the intervention involved direct work with the children in both the "traditional parent training program" and the "STEPP"

Chronis 2004 Intervention study involving children with ADHD using a BAB design to assess effects of delivery then withdrawal of a behavioural modification programme involving direct work with the children. No true control group

Chronis 2006

RCT involving mothers of children with ADHD, a population known to be at risk of depression. The 'Coping With Depression Course' was not assessed to meet inclusion criteria for parent training. Child behaviour was, however, assessed, as well as maternal functioning, and ADHD-related family impairment

Connell 1997

RCT of oppositional preschoolers to parent training or waitlist control. Excluded for both age and lack of ADHD diagnosis

Corkum 1999

RCT of methylphenidate plus parent training versus methylphenidate plus parent support. No outcomes for children. Additional note: investigators confirmed PT and PS support, attendance was very low Schachar 1997 supplies additional information concerning this study

Corkum 2005

RCT involving children diagnosed with ADHD DSM-IV-TR and aged between 5-12 years old. Both interventions were active (parent training verus parent training combined with teacher support) this therefore does not meet inclusion criteria

Corrin 2003

RCT wherein children with a 'younger cohort' of children (aged from 4 years up); not all diagnoses secure. Both active interventions involved direct work with children (child training alone was compared with parent plus child training). No parent training alone; no no-treatment control 
Coughlin $2009 \quad$ Controlled (and possibly randomised) trial of children with "significant behavioural problems" but not necessarily an ADHD diagnosis, within an intervention or TAU group. The intervention group was flexible, involving a video-modelling treatment including parent training but also direct work with children at times (thus not meeting this inclusion criterion as well)

Cummings 2008

RCT of children aged 26-72 months without formal diagnoses of ADHD, allocated to one of two active parent training groups which therefore does not meet inclusion criteria due to diagnosis, age and lack of eligible control group

Danforth 1998

Uncontrolled intervention study of children with ODD and/or ADHD using a multiple baseline design

Dubbs 2008

Intervention study involving direct work with children without formal diagnosis of ADHD with children part of intervention

Dubey 1978

This early paper (Dubey 1978) described "six clinical programs and one controlled, experimental program". The latter was a small RCT; however, participants had been recruited without a formal ADHD diagnosis, using only the Werry Weiss Peters scale, a screening measure with low sensitivity and lacking measures of impairment (regarded as insufficient for secure diagnosis (Daley 2009). Dubey 1983 reports on a subset of data from the original paper

Ellis 2009

Not an intervention study but a study looking at parents of children with ADHD and considering parents' own ADHD symptoms in relation to their parenting practices

Ercan 2005

Intervention study of children with ADHD plus CD or ADHD plus ODD involving combined modality treatment (parent training plus methylphenidate) which was not randomised or even quasi-randomised (participants self selected into treatment and control groups)

Fabiano 2006

RCT of children with ADHD; participants were randomised to either parent training for fathers only or parent training plus sports activities for fathers and children. Although a de facto wait list control group was created, recruitment was not contemporaneous and therefore not part of the original randomisation (note: principal investigator noted with disappointment his ethics committee's refusal to allow him to create a contemporaneous no-treatment control group)

Fagan Rogers $2009 \quad$ Not an intervention study but an investigation of the role of parental involvement in children's academic progress

Gibbs 2008

Controlled before and after intervention involving parents who chose (or chose not to) participate in a parenting programme whilst their children (diagnosed with a range of disruptive and emotional disorders but not necessarily ADHD) attended a health camp where a psychosocial intervention was delivered. This study is excluded both for reasons of sequence generation (self selection) and lack of adequate diagnosis

Grimm 2006

RCT of children with conduct problems (mixed, not all with ADHD) with three active treatment arms, each a variant of a parenting programme ( no no-treatment control group)

Guo 2008

RCT conducted in China of children with ADHD. Translation indicates that the intervention involved parent training in combination 'family meetings' (which appear to have involved a chance to share experiences and 'express emotions') as well as home visits during which clinicians engaged in direct work with the children. Study excluded because of direct work with the children 

not available so excluded on the basis of no formal diagnosis of ADHD (author confirmed that separate data were not obtainable)

Hall 2003

RCT involving children with ADHD, excluded because of the three arms (child training only, child/parent training and child/parent training plus home/school-based behavioural consultation) none involved an eligible 'no treatment' group

Hauth-Charlier 2009 Review article; not an intervention study

Hautmann 2009 An intervention study, but with no control group. Inclusion criteria "did not depend on meeting a defined threshold of symptom severity" but simply that a child over the age of three had an externalising problem

Heriot 2008

RCT of $0.3 \mathrm{mg} / \mathrm{kg}$ methylphenidate + parent training programme versus $0.3 \mathrm{mg} / \mathrm{kg}$ methylphenidate + parent support group versus placebo + parent training programme versus placebo + parent support group. Participants were diagnosed with ADHD (DSM-IV) based on rating scales completed by parents and teachers rather than clinicians and also slightly too young for inclusion within this review - range 3.0-5.9 years, mean $=4.77$

Horn 1991

RCT involving children ADHD comparing high and low doses of methylphenidate alone and in combination with behavioural parent plus child self control instruction. Study excluded because of direct work with children in parent training arm and lack of an adjunctive or no treatment arm

Isler 2003

RCT of children with formal diagnosis of ADHD; however, study lacks eligible control group. All interventions were 'active': participants were randomised either to a child group training or a parent training plus child training plus home and school training. This therefore does not meet inclusion criteria as there is no "no treatment control group" and both interventions involved direct work with children

Jones 2008

RCT of parent training group vs waiting list. Excluded due to children being underage (36-48 months) and lacking secure diagnoses of ADHD

Larsson 2008

RCT design acceptable; parent training programme and controls acceptable; outcomes acceptable. Diagnosis remained difficult to assess, even after personal communication with investigators and after reading multiple publications. According to an early publication, participants were "those who received a possible or definitive diagnosis of ODD and/or CD after assessment after all clinically referred children were first screened by means of the Eyberg Child Behavior Inventory (ECBI) using the 90th percentile as a cut-off score according to Norwegian norms. Children who attained such a cut-off score or higher were subsequently interviewed by one of three trained interviewers using the KIDDIE-SADS” (Drugli, p 393) . Subsequent contact with Dr Drugli suggested that subset ADHD children were similarly diagnosed (i.e. by trained interviewers but not specialists). In the paper published by Larsson et al (2008) authors report subset for "definitive" ADHD participants; but in the paper by Fossum et al (2008) authors admit as a limitation of the study that "the assessment of clinical levels of ADHD did not meet the formal criteria of a diagnosis."

Lauth 2007

RCT with three arms of children with behavioural problems, a subset of whom had ADHD (data not reported separately). The trial compared parent training with parent training combined with behavioural training for children compared with a parent support group in which "emotional and social themes" identical to those in the other groups were discussed. After obtaining a partial translation of the paper we 
adjudged that the latter group was more than an 'attentional control' (as other similar groups had been constructed in other studies) in that a 'script' of behavioural issues, mapping on to the training in other groups, had been provided. ADHD was in addition not the focus of the study

Lavigne 2008

Three-armed RCT focusing on very young children with a diagnosis of OCD. Participants were too young for this review: "Study participants were children ages 3.0-6.11 years and their parents" (average age 4.6 years, SD 1/4 1.0)

Markie-Dadds 2006 RCT with three arms (enhanced self-directed behavioural family intervention, a self help program and a waitlist control) for children with conduct problems (not ADHD specifically). Children were aged 2 to 6 years (mean 3.9)

McGoey $2005 \quad$ RCT of an early intervention group versus a community treatment group (which may have involved parent training). Child participants were aged 3 to 5 years and 'at risk' for ADHD, which does not meet inclusion criteria

Miranda 2006 Study was quasi-experimental and not randomised. A pharmacological intervention (methylphenidate) was compared to a psychosocial intervention (programme in the classroom - excluded because intervention involved direct work with children without formal diagnosis of ADHD) versus a control group

Molina 2008

RCT. Participants (middle-school children diagnosed with ADHD) were randomised to a 10 week programme or a community comparison. Intervention does not meet inclusion criteria as it involved direct work with the children

Montiel 2002

RCT. Participants "diagnosed as having ADHD, identified in ADHD screening days" were randomised to parent training or medication group; does not meet intervention inclusion criteria as no comparison of parent training versus no parent training group

Morawska 2009

RCT of Triple-P Positive Training programme versus a waitlist control. Children involved may have had behavioural problems and outcomes included hyperactivity, but children did not necessarily have ADHD; entry criteria specified only that they be identified as "gifted"

MTA 1999 Complex large scale RCT; intervention included direct work with the children:

"Behavioral treatment included parent training, child- focused treatment, and a school-based intervention organized and integrated with the school year. The parent training, based on work by Barkley and Forehand and MacMahon,37 involved 27 group ( 6 families per group) and 8 individual sessions per family. It began weekly on randomization, concurrent with biweekly teacher consultation; both were tapered over time. The same therapist- consultant conducted parent training and teacher consultation, with each therapistconsultant having a case- load of 12 families." (pp 1074-1075)

Nixon 2001

RCT involving children too young for inclusion in this review (aged 3 to 5 years) with behavioural disturbances were randomised to an intervention involving both parent training in behavioural management and direct work with children ('parent-child interaction therapy' or PCIT), versus wait list control, compared with a 'nondisturbed' preschool sample

Nixon 2003

RCT of PCIT (see above) where child participants had ODD with no diagnosis of ADHD and, as above, intervention involved direct work with children 

agement group or a control group. Children were included if they had extreme scores greater or equal to 15 on Connors teacher rating scale, which does not meet diagnosis inclusion criteria for ADHD

Odom 1996

RCT of what was described as a primarily "educational intervention" compared to a no treatment control group. Participants were mothers of children aged 5 to 11 years diagnosed with ADHD by an MDT evaluation. No child outcomes were measured, only those of the mother (knowledge of ADHD, willingness to have their child medicated and willingness to seek counselling, parenting sense of competence) were reported, which does not meet inclusion criteria

Pisterman 1989

RCT of eligible parent training intervention versus control; however, participants included parents of children aged between 3-6 years without formal ADHD diagnosis (diagnosis made by parent structured screening interview by $\mathrm{PhD}$ psychologist) which does not meet inclusion criteria (Pisterman 1992b reports follow-up)

Pisterman 1992a

RCT of eligible parent training intervention versus control; however, participants included parents of children aged between 46.42-52.41 months, and again without formal ADHD diagnosis (diagnosis made by parent or teacher on SNAP checklist (Pelham 1982) which does not meet inclusion criteria (Pisterman 1992b reports follow-up)

Pollard 1983

Pre-post design of both methylphenidate and parent training on the behaviour of three 'hyperkinetic boys'

Reeves 2009

Not a randomised controlled trial. Review article

Salbach 2005

Controlled study, but neither randomised or quasi-randomised, comparing parent training plus medication (methylphenidate) plus consultation versus medication plus consultation alone for parents of children with ADHD. Excluded because participants chose their intervention groups themselves

Sanders 2000a

RCT. Participants were randomised to enhanced behavioural family intervention, standard behavioural family intervention, self-directed behavioural family intervention or wait list control. Participants had no formal diagnosis of ADHD. Participants were aged 3 years old (between 36 and 48 months - mean age was 3.39yrs) which does not meet inclusion criteria. McLennan 2001 summarises results of this study

Sanders $2000 \mathrm{~b}$

RCT. Participants were randomly assigned to a behavioural family intervention or cognitive behavioural family intervention which does not meet inclusion criteria as there is no eligible control group. Participants had no formal ADHD diagnosis, only 2 children had ADHD based on a structured interview with the mother using DSM-IV criteria. Participants were aged 3-9 years $($ mean $=4.39)$ which does not meet inclusion criteria

Scahill 2006

RCT of parent training versus no treatment control. Focus of study was not ADHD, but disruptive behaviour in children with tics. Investigators recruited children with comorbid tic and disruptive behaviour disorders from a specialised tic disorders clinic. They specifically excluded children with ADHD not receiving medication. This yielded a subset of children with comorbid, medicated ADHD

Schoppe-Sullivan 2009 Not a randomised controlled trial (although indexed in MEDLINE as such). Observational study investigated how co-parenting affected children's externalizing behaviour and attempts at "effortful control", as rated by children's teachers and mothers 
Multicentre RCT involving parenting groups for children who were recruited for antisocial behaviour rather than ADHD. "Eligible children were all those aged 3-8 years who were referred for antisocial behaviour to their local multidisciplinary child and adolescent mental health service" (p 2). From the text, it would appear investigators strenuously sought to exclude ADHD, as they listed as exclusion criteria for their trial: "clinically apparent major developmental delay, hyperkinetic syndrome [ICD-10 criteria for inclusion within this review] or any other condition requiring separate treatment". ADHD is not mentioned in the published study. Personal contact with the author (Scott 2011) concerning a different study (Scott 2010) led to a disclosure that approximately half the study's participants subsequently proved to meet diagnostic criteria for ADHD (although the age of such children remains unclear) and data were generously provided. However, due to concerns that because ADHD was far from being the focus of this study (wherein recruitment included only aggressive children and [initially at least] attempted to excluded any child with a diagnosis or treatment for $\mathrm{ADHD}$ ), we decided these data do not meet inclusion criteria

RCT involving a mixed intervention programme including aspects of Webster-Stratton's Incredible Years and aspects of the SPOKES projects in which parents read with their children, to promote literacy. Participants (all aged 6 years) were screened for a range of risk factors for antisocial behaviour, low reading ability, conduct problems and 'ADHD symptoms' via the PACS. Thus, a true diagnosis for ADHD of children was not made (nor was it the focus of the intervention)

Sonuga-Barke 2001 RCT wherein participants were randomised to parent training, parent counselling and support or wait list control. Children were 3 years old, which does not meet inclusion criteria. Participants had no formal diagnosis of ADHD, diagnoses was based on scores on WWP and PACS, which does not meet inclusion criteria. No child outcomes, which does not meet inclusion criteria

Baldwin 2001 summarises aspects of this study and Sonuga Barke 2002 provides additional data

Sonuga-Barke 2004 RCT wherein participants were randomised to parent training or wait list control. Children were 3 years old, which does not meet inclusion criteria. Participants were diagnosed with 'preschool ADHD' which does not meet inclusion criteria

Springer 2004 RCT with three active intervention arms, all involving direct work with the child. Age range and diagnosis of ADHD acceptable

Taylor 1998

Controlled but not randomised nor quasi-randomised study comparing Webster-Stratton's Parents and Children Series parenting groups, the eclectic approach treatment or wait list control. Allocation not randomised, investigators wrote, in order "to allow urgent families, and families who had already waited a long time for treatment, to remain in the study". Children had behavioural issues but not necessarily a diagnosis of ADHD, aged 3-8 years old

Treacy 2005

RCT focused on parental stress alone, in which participants were randomly assigned to parent stress management training or wait list control. Children were diagnosed with DSM-IV ADHD. Children were aged 6-15 years

No outcomes involved children. Outcomes measured included only Parenting Stress Index (PSI) (Abidin 1995) Parent Scale (Arnold 1993), Parental Locus of Control Scale (PLOC) (Campis 1986)

van der Oord 2008

RCT wherein participants with ADHD were randomised to methylphenidate or methylphenidate plus behaviour therapy. There was a direct clinical intervention involving the children: "The multimodal behavior therapy integrated family based and school-based interventions with cognitive behavior therapy of the child" (p 50) 
(Continued)

Waschbusch 2005 Cluster RCT targeting disruptive children. Diagnosis of ADHD was unclear for all children and all active interventions ('universal' school wide intervention; targeted school intervention; targeted home intervention; control group) involved direct work with the child

Weinberg $1999 \quad$ Single group intervention study (pre-post test measures) of parent training for parents of children with ADHD. No control group

Wolraich 2005

RCT in which participants (mean age 7.41 , of whom only a portion had a secure ADHD diagnosis) were randomly assigned to a treatment or a control group, however the intervention (which focused on improving communication between parents, teachers and primary care providers) and did not meet inclusion criteria as the treatment group did not consist of true parent training 
DATA AND ANALYSES

Comparison 1. Parent training versus control

\begin{tabular}{lcclc} 
Outcome or subgroup title & $\begin{array}{c}\text { No. of } \\
\text { studies }\end{array}$ & $\begin{array}{c}\text { No. of } \\
\text { participants }\end{array}$ & \multicolumn{1}{c}{ Statistical method } & Effect size \\
\hline $\begin{array}{l}1 \text { 1 Child's ADHD behaviour } \\
\text { (home setting) CPRS-R:S }\end{array}$ & 1 & 96 & Mean Difference (IV, Random, 95\% CI) & $0.30[-2.50,3.10]$ \\
$\begin{array}{l}\text { 2 2a Externalising } \\
\text { 3 2b Internalising }\end{array}$ & 3 & 174 & Std. Mean Difference (IV, Random, 95\% CI) & $-0.32[-0.83,0.18]$ \\
$\begin{array}{l}4 \text { 7 Parenting stress - PSI - parent } \\
\quad \text { domain }\end{array}$ & 2 & 142 & Std. Mean Difference (IV, Random, 95\% CI) & $-0.48[-0.84,-0.13]$ \\
$\begin{array}{l}5 \text { 7 Parenting stress PSI - child } \\
\text { domain }\end{array}$ & 2 & 142 & Mean Difference (IV, Random, 95\% CI) & $-7.54[-24.38,9.30]$ \\
\hline
\end{tabular}

\section{Analysis I.I. Comparison I Parent training versus control, Outcome I I Child's ADHD behaviour (home setting) CPRS-R:S.}

Review: Parent training interventions for Attention Deficit Hyperactivity Disorder (ADHD) in children aged 5 to 18 years

Comparison: I Parent training versus control

Outcome: I I Child's ADHD behaviour (home setting) CPRS-R:S

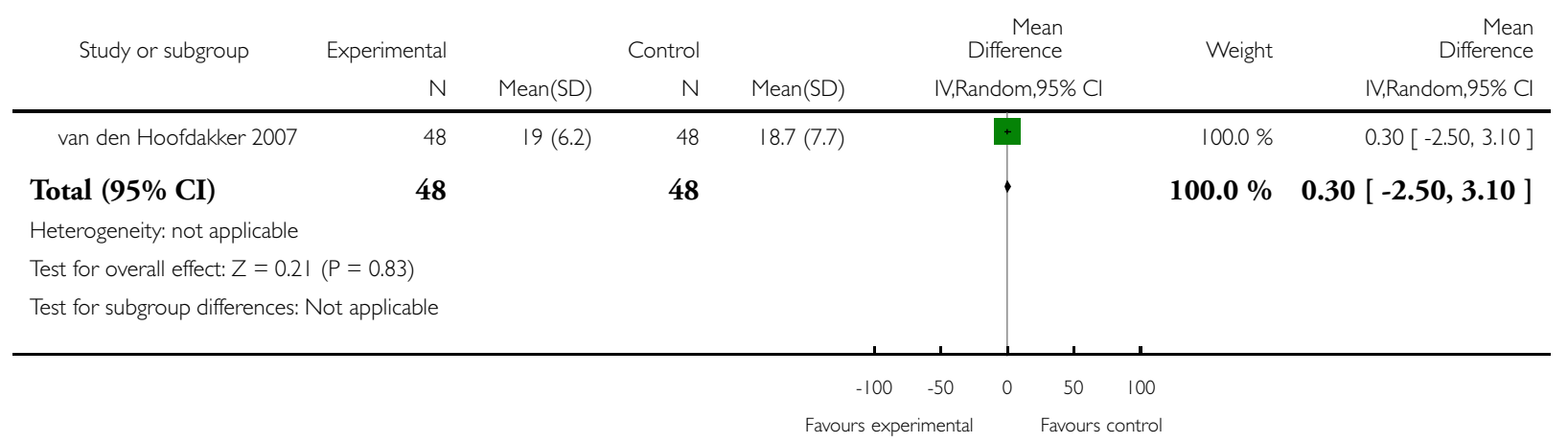




\section{Analysis I.2. Comparison I Parent training versus control, Outcome 2 2a Externalising.}

Review: Parent training interventions for Attention Deficit Hyperactivity Disorder (ADHD) in children aged 5 to 18 years

Comparison: I Parent training versus control

Outcome: 2 2a Externalising

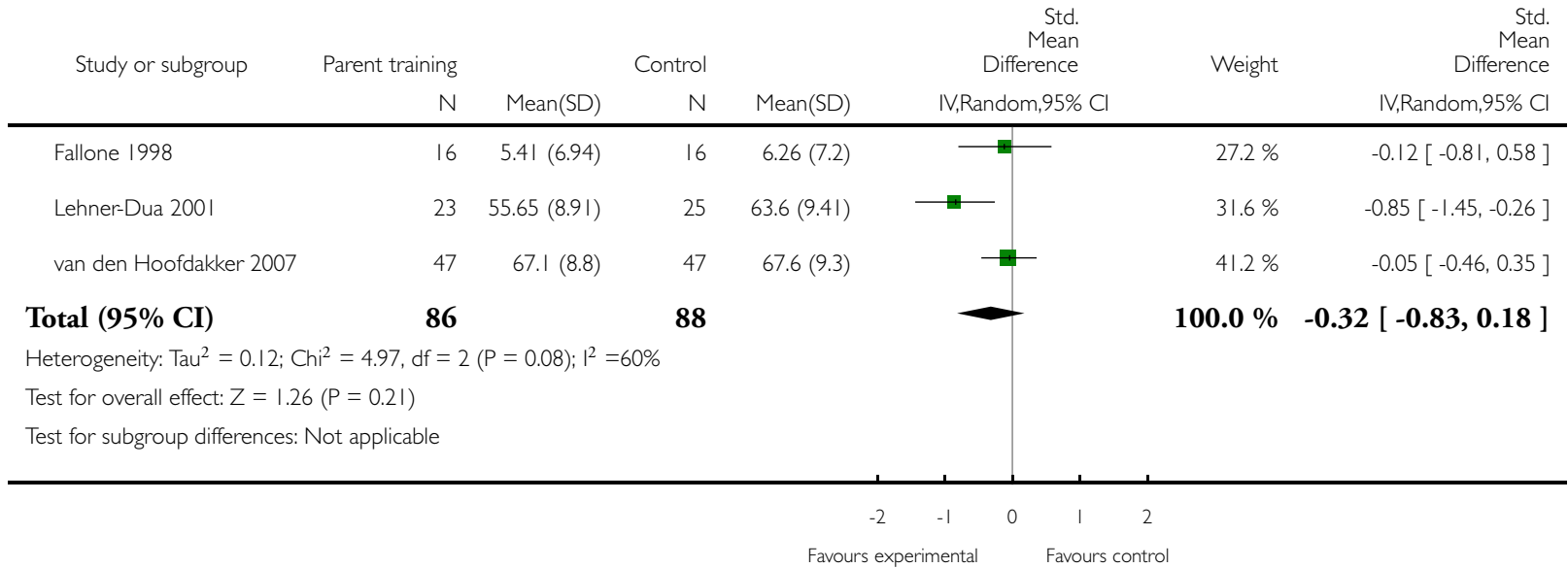

\section{Analysis I.3. Comparison I Parent training versus control, Outcome 32 b Internalising.}

Review: Parent training interventions for Attention Deficit Hyperactivity Disorder (ADHD) in children aged 5 to 18 years

Comparison: I Parent training versus control

Outcome: $32 \mathrm{~b}$ Internalising

\begin{tabular}{|c|c|c|c|c|c|c|c|c|}
\hline \multirow[t]{2}{*}{ Study or subgroup } & Parenting training & \multicolumn{2}{|r|}{ Control } & \multicolumn{3}{|c|}{$\begin{array}{r}\text { Std. } \\
\text { Mean } \\
\text { Difference }\end{array}$} & \multirow[t]{2}{*}{ Weight } & \multirow{2}{*}{$\begin{array}{r}\text { Std. } \\
\text { Mean } \\
\text { Difference } \\
\text { IV,Random,95\% Cl }\end{array}$} \\
\hline & N & Mean(SD) & $N$ & Mean(SD) & \multicolumn{2}{|c|}{ IV,Random,95\% Cl } & & \\
\hline Lehner-Dua 200। & 23 & $46.7(7.79)$ & 25 & $52.84(8.57)$ & $\longleftarrow$ & & $34.1 \%$ & $-0.74[-1.32,-0.15]$ \\
\hline van den Hoofdakker 2007 & 47 & $60.3(10.2)$ & 47 & $64(10.5)$ & $\rightleftarrows$ & & $65.9 \%$ & $-0.35[-0.76,0.05]$ \\
\hline Total $(95 \%$ CI) & 70 & & 72 & & & & $100.0 \%$ & $-0.48[-0.84,-0.13]$ \\
\hline \multicolumn{9}{|c|}{ Heterogeneity: $\operatorname{Tau}^{2}=0.01 ; \mathrm{Chi}^{2}=1.09, \mathrm{df}=\mathrm{I}(\mathrm{P}=0.30) ; \mathrm{I}^{2}=9 \%$} \\
\hline \multicolumn{9}{|c|}{ Test for overall effect: $Z=2.68(P=0.0074)$} \\
\hline \multicolumn{9}{|c|}{ Test for subgroup differences: Not applicable } \\
\hline & & & & & -0.5 & 0.5 & । & \\
\hline \multicolumn{9}{|c|}{ Favours experimental } \\
\hline
\end{tabular}

Parent training interventions for Attention Deficit Hyperactivity Disorder (ADHD) in children aged 5 to 18 years (Review)

Copyright ( 201 I The Cochrane Collaboration. Published by John Wiley \& Sons, Ltd. 


\section{Analysis I.4. Comparison I Parent training versus control, Outcome 47 Parenting stress - PSI - parent} domain.

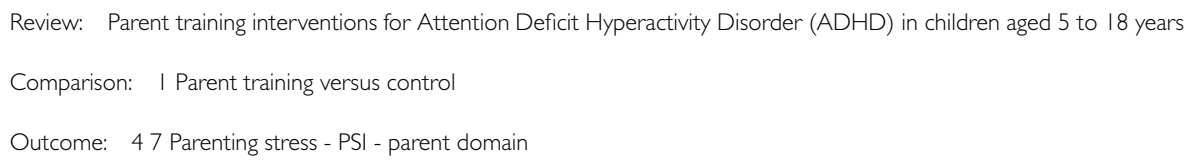

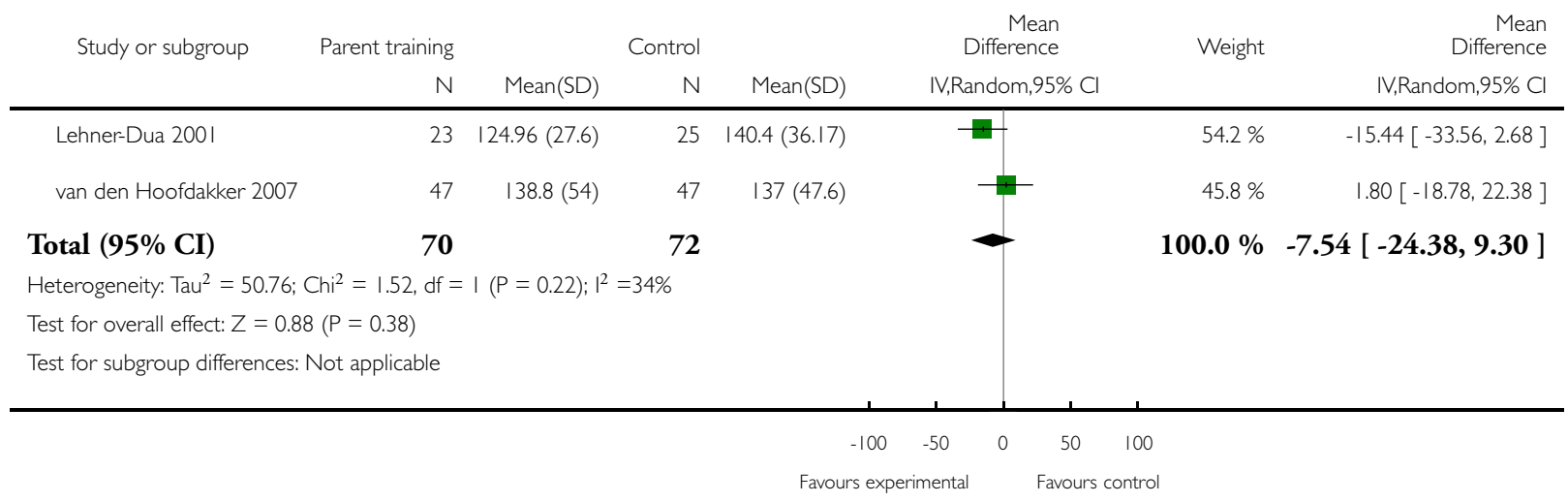




\section{Analysis I.5. Comparison I Parent training versus control, Outcome 57 Parenting stress PSI - child domain.}

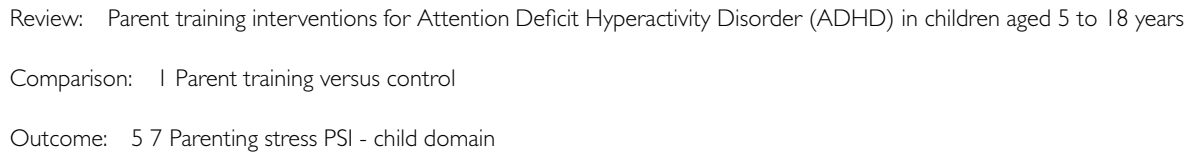

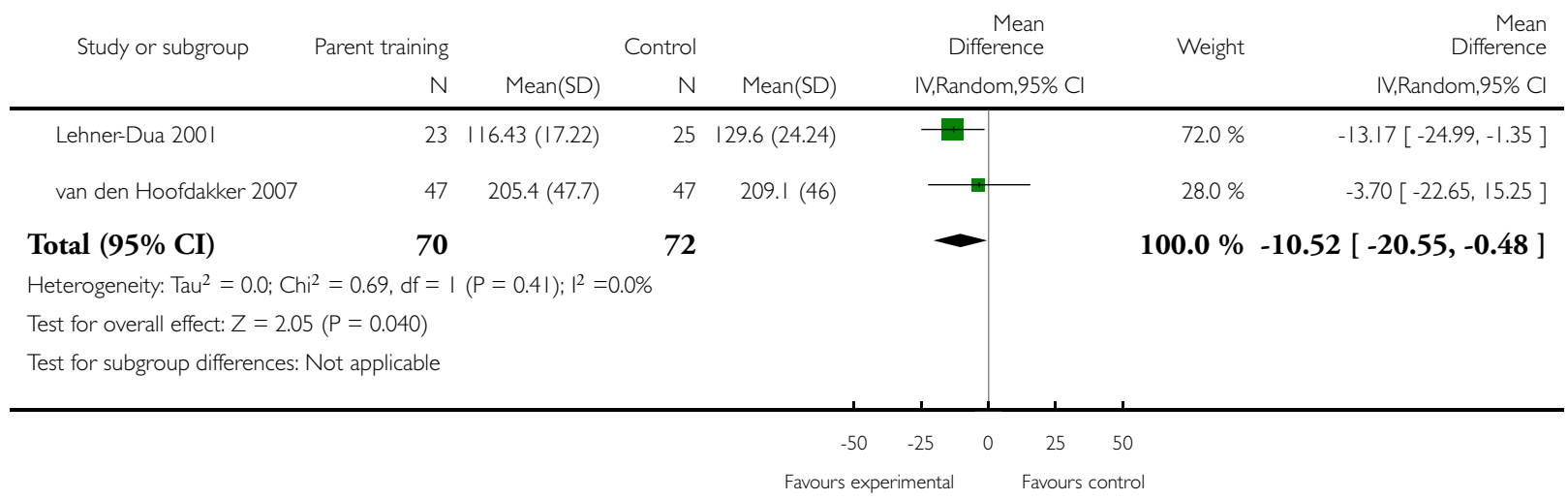

\section{A P P E N DICES}

\section{Appendix I. Search strategies 2002, 2004, 2006, 2008}

1. Cochrane Central Register of Controlled Trials (CENTRAL) (2002-2008) and the National Research Register (until 2007) \#1 MeSH descriptor Psychotherapy explode all trees

\#2 MeSH descriptor Family Relations explode all trees

\#3 (parent* or famil ${ }^{*}$ or father* or mother* or paternal* or maternal* or couple* or marital ${ }^{*}$ )

\#4 (psycho* therap*)

\#5 (behavio* near therap*)

\#6 behavio* $^{*}$ near intervention*

\#7 behavio* near treatment ${ }^{*}$

\#8 multimodal $^{*}$ or multi-modal*

\#9 (mta)

\#10 (parent* near program*)

\#11 parent* near train*

\#12 parent* $^{*}$ near educat ${ }^{*}$

\#13 parent* near promot*

\#14 parent-train*

\#15 parent-educat*

\#16 parent-promot*

\#17 parent* near therap* $^{*}$

\#18 (\#1 OR \#2 OR \#3 OR \#4 OR \#5 OR \#6 OR \#7 OR \#8 OR \#9 OR \#10 OR \#11 OR \#12 OR \#13 OR \#14 OR \#15 OR \#16 OR \#17) 
\#19 MeSH descriptor Attention Deficit and Disruptive Behavior Disorders explode all trees

\#20 attention near deficit

\#21 child* near attention

\#22 child* near inattention

\#23 child* near impulsiv*

\#24 child* near overactiv*

\#25 hyperkin*

\#26 hyper near activ*

\#27over near activ*

\#28 hyper near kin*

\#29 hyperactiv*

\#30 disruptiv*

\#31 adhd or addh

\#32 ad next hd

\#33 minimal next brain

\#34 brain and dysfunction

\#35(\#19 OR \#20 OR \#21 OR \#22 OR \#23 OR \#24 OR \#25 OR \#26 OR \#27 OR \#28 OR \#29 OR \#30 OR \#31 OR \#32 OR \#33

OR\#34)

\#36 (\#18 AND\#35)

2. Search strategy for MEDLINE, EMBASE and CINAHL (all via Ovid)

exp PSYCHOTHERAPY/

exp Family Relations/

(parent $\$$ or famil $\$$ or father $\$$ or mother $\$$ or paternal $\$$ or maternal $\$$ or couple $\$$ or marital $\$$ ).mp

(psycho\$ adj therap\$).mp

(behavio\$ adj5 therap\$).mp

(behavio\$ adj5 intervention\$).mp

(behavio\$ adj5 treatment\$).mp

(multimodal\$ or multi-modal\$).mp

MTA.mp.

(parent\$ adj3 program\$).mp

(parent $\$$ adj3 train $\$$ ).mp

(parent\$ adj3 educat\$).mp

(parent\$ adj3 promot\$).mp.

parent-train $\$ . m p$

parent-educat $\$ . m p$.

parent-promot\$.mp.

(parent $\$$ adj3 therap\$).mp.

1 or 2 or 3 or 4 or 5 or 6 or 7 or 8 or 9 or 10 or 11 or 12 or 13 or 14 or 15 or 16 or 17

exp Attention Deficit Disorder with Hyperactivity/

(attention adj3 deficit).mp.

(child $\$$ adj 3 attention).mp.

(child $\$$ adj3 inattention).mp.

(child\$ adj3 impulsiv\$).mp.

(child\$ adj3 overactiv\$).mp.

hyperkin \$.mp.

(hyper adj3 activ\$).mp.

(over adj3 activ\$).mp.

(hyper adj3 kin\$).mp.

hyperactiv\$.mp.

disruptiv\$.mp.

(adhd or addh).mp.

(ad hd or ad??hd).mp.

33 (minimal adj brain).mp.

Parent training interventions for Attention Deficit Hyperactivity Disorder (ADHD) in children aged 5 to 18 years (Review)

Copyright (c) 201 I The Cochrane Collaboration. Published by John Wiley \& Sons, Ltd. 
34 (brain and dysfunction).mp.

3519 or 20 or 21 or 22 or 23 or 24 or 25 or 26 or 27 or 28 or 29 or 30 or 31 or 32 or 33 or 34

$36 \quad 18$ and 35

Appropriate RCT filters were added for each database.

3. Search strategy for PsycINFO via SilverPlatter

((parent* near educat*) or (( parent* near program* $)$ or ( parent* near train* $)$ or ${ }^{*}$ parent* near promot* $\left.)\right)$ or ("Parent-Training" in MJ,MN) or (( parent-train*) or ( parent-educat* $)$ or ( parent-promot* $))$ and $((($ minimal brain*) or (hyperkinesis*) or (hyperactiv*) or (attention near3 deficit) or (adhd or addh) or ("Attention-Deficit-Disorder-with-Hyperactivity" in MJ,MN))

The results were then put into a Procite database and searched again using the terms:

(random* OR trial* OR crossover* OR blind* OR placebo*)

4. Search strategy for Dissertation Abstracts International searched via Dissertation Express

(adhd OR addh OR attention deficit OR minimal brain dysfunction OR hyperkinetic syndrome)

5. ClinicalTrial.gov (last searched 6 November 2006)

(adhd OR addh OR attention deficit OR minimal brain dysfunction OR hyperkinetic syndrome)

\section{Appendix 2. Search strategies used May 2009}

1. Cochrane Central Database of Controlled Trials (CENTRAL) 2009 Issue 2 (searched 20 May 2009)

\#1 MeSH descriptor Psychotherapy explode all trees

\#2 MeSH descriptor Family Relations explode all trees

\#3 (parent* or famil* or father* or mother* or paternal* or maternal* or couple* or marital*)

\#4 (psycho* therap*)

\#5 (behavio* near therap*) $^{*}$

\#6 behavio* near intervention*

\#7 behavio* near treatment*

\#8 multimodal ${ }^{*}$ or multi-modal*

\#9 (mta)

\#10 (parent* near program*)

\#11 parent* $^{*}$ near train*

\#12 parent* $^{*}$ near educat*

\#13 parent* near promot*

\#14 parent-train*

\#15 parent-educat*

\#16 parent-promot*

\#17 parent* near therap* $^{*}$

\#18 (\#1 OR \#2 OR \#3 OR \#4 OR \#5 OR \#6 OR \#7 OR \#8 OR \#9 OR \#10 OR \#11 OR \#12 OR \#13 OR \#14 OR \#15 OR \#16

OR \#17)

\#19 MeSH descriptor Attention Deficit and Disruptive Behavior Disorders explode all trees

$\# 20$ attention near deficit

\#21 child* near attention

\#22 child* near inattention

\#23 child* near impulsiv*

\#24 child* near overactiv*

\#25 hyperkin*

\#26 hyper near activ*

\#27over near activ*

\#28 hyper near kin*

\#29 hyperactiv*

\#30 disruptiv*

\#31 adhd or addh

\#32 ad next hd

\#33 minimal next brain

Parent training interventions for Attention Deficit Hyperactivity Disorder (ADHD) in children aged 5 to 18 years (Review)

Copyright (C) 201 I The Cochrane Collaboration. Published by John Wiley \& Sons, Ltd. 
\#34 brain and dysfunction

\#35(\#19 OR \#20 OR \#21 OR \#22 OR \#23 OR \#24 OR \#25 OR \#26 OR \#27 OR \#28 OR \#29 OR \#30 OR \#31 OR \#32 OR \#33

OR \#34)

\#36 (\#18 AND\#35)

2.CINAHL via Ovid (searched 20 May 2009)

Psychotherapy.mp. or exp PSYCHOTHERAPY/

2 family relations.mp. or exp Family Relations/

(parent $\$$ or famil $\$$ or father $\$$ or mother $\$$ or paternal $\$$ or maternal $\$$ or couple $\$$ or marital $\$$ ).mp.

(psycho\$ adj therap\$).mp.

(behavio \$ adj5 therap\$).mp.

(behavio\$ adj5 intervention\$).mp.

(behavio\$ adj5 treatment\$).mp.

(multimodal\$ or multi-modal\$).mp.

MTA.mp.

10 (parent\$ adj3 program\$).mp.

11 (parent $\$$ adj3 train $\$$ ).mp.

12 (parent $\$$ adj3 educat\$).mp. [mp=title, subject heading word, abstract, instrumentation] (4166)

13 (parent $\$$ adj3 promot\$).mp.

14 parent-train $\$ . m p$.

15 parent-educat\$.mp.

16 parent-promot\$.mp.

17 (parent\$ adj3 therap\$).mp.

181 or 2 or 3 or 4 or 5 or 6 or 7 or 8 or 9 or 10 or 11 or 12 or 13 or 14 or 15 or 16 or 17

$19 \exp$ Attention Deficit Disorder with Hyperactivity/

20 (attention adj3 deficit).mp.

21 (child $\$$ adj3 attention).mp.

22 (child $\$$ adj 3 inattention).mp.

23 (child\$ adj3 impulsiv\$).mp.

24 (child\$ adj3 overactiv\$).mp.

25 hyperkin \$.mp.

26 (hyper adj3 activ\$).mp.

27 (over adj3 activ\$).mp.

28 (hyper adj3 kin\$).mp.

29 hyperactiv\$.mp.

30 disruptiv\$.mp.

31 (adhd or addh).mp.

32 (ad hd or ad??hd).mp.

33 (minimal adj brain).mp.

34 (brain and dysfunction).mp.

3519 or 20 or 21 or 22 or 23 or 24 or 25 or 26 or 27 or 28 or 29 or 30 or 31 or 32 or 33 or 34

$36 \quad 18$ and 35

37 randomi\$.mp.

38 clin\$.mp.

39 trial\$.mp.

$40 \quad(\operatorname{clin} \$$ adj3 trial\$).mp.

41 singl\$.mp.

42 doubl\$.mp.

43 tripl\$.mp.

44 trebl\$.mp.

45 mask\$.mp.

46 blind $\$ . m p$.

47 (41 or 42 or 43 or 44$)$ and (45 or 46)

48 crossover.mp.

Parent training interventions for Attention Deficit Hyperactivity Disorder (ADHD) in children aged 5 to 18 years (Review)

Copyright $(2011$ The Cochrane Collaboration. Published by John Wiley \& Sons, Ltd. 


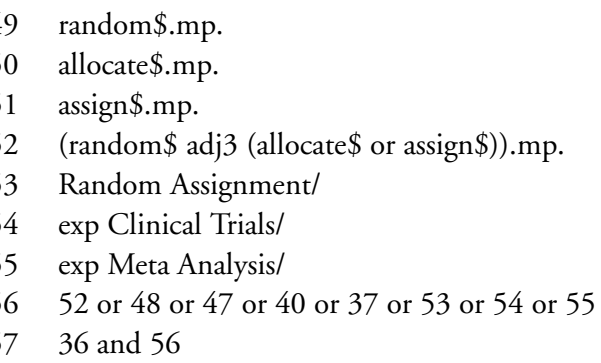

\section{Search Strategy for EMBASE (searched Week 22 2009)}

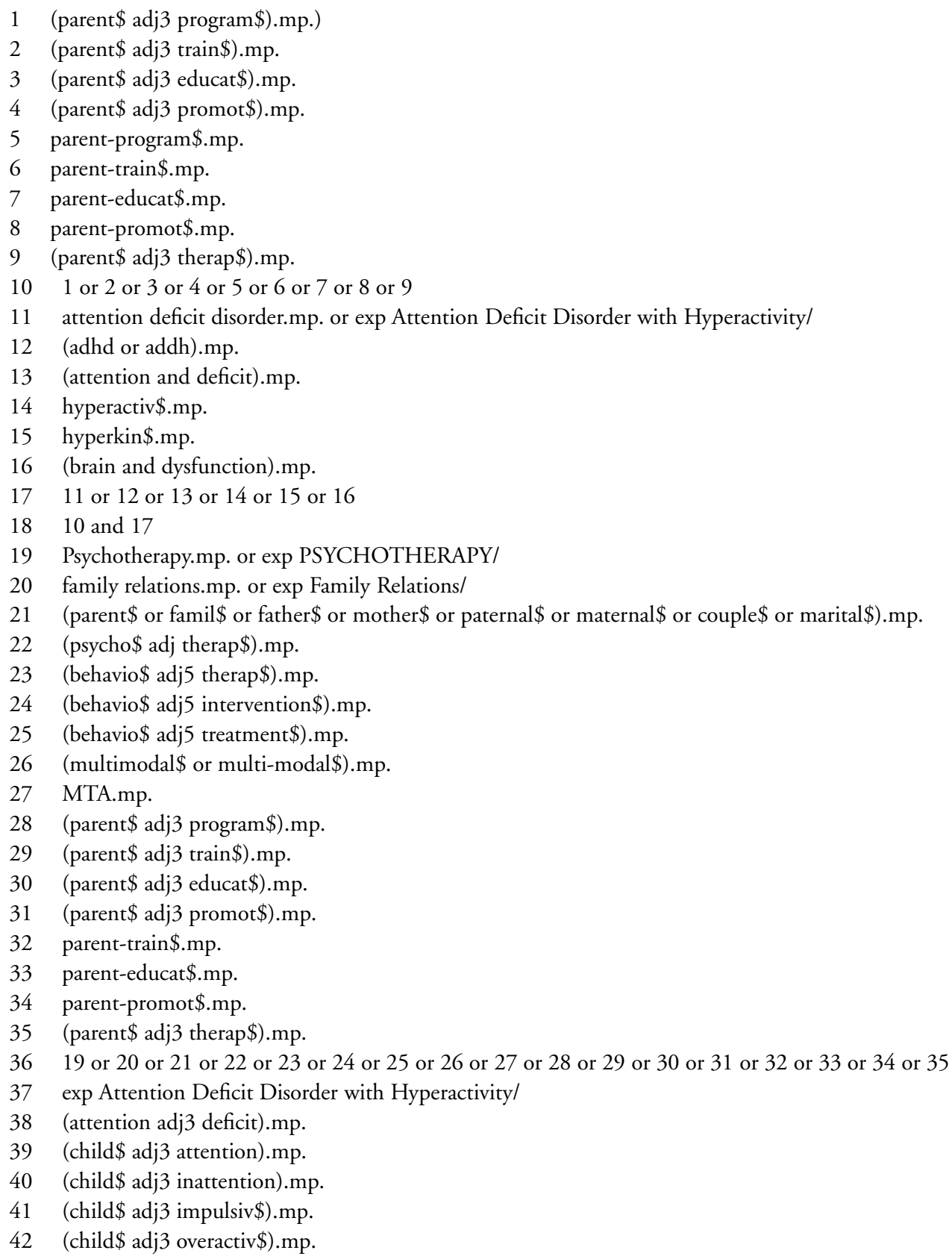

Parent training interventions for Attention Deficit Hyperactivity Disorder (ADHD) in children aged 5 to 18 years (Review) 


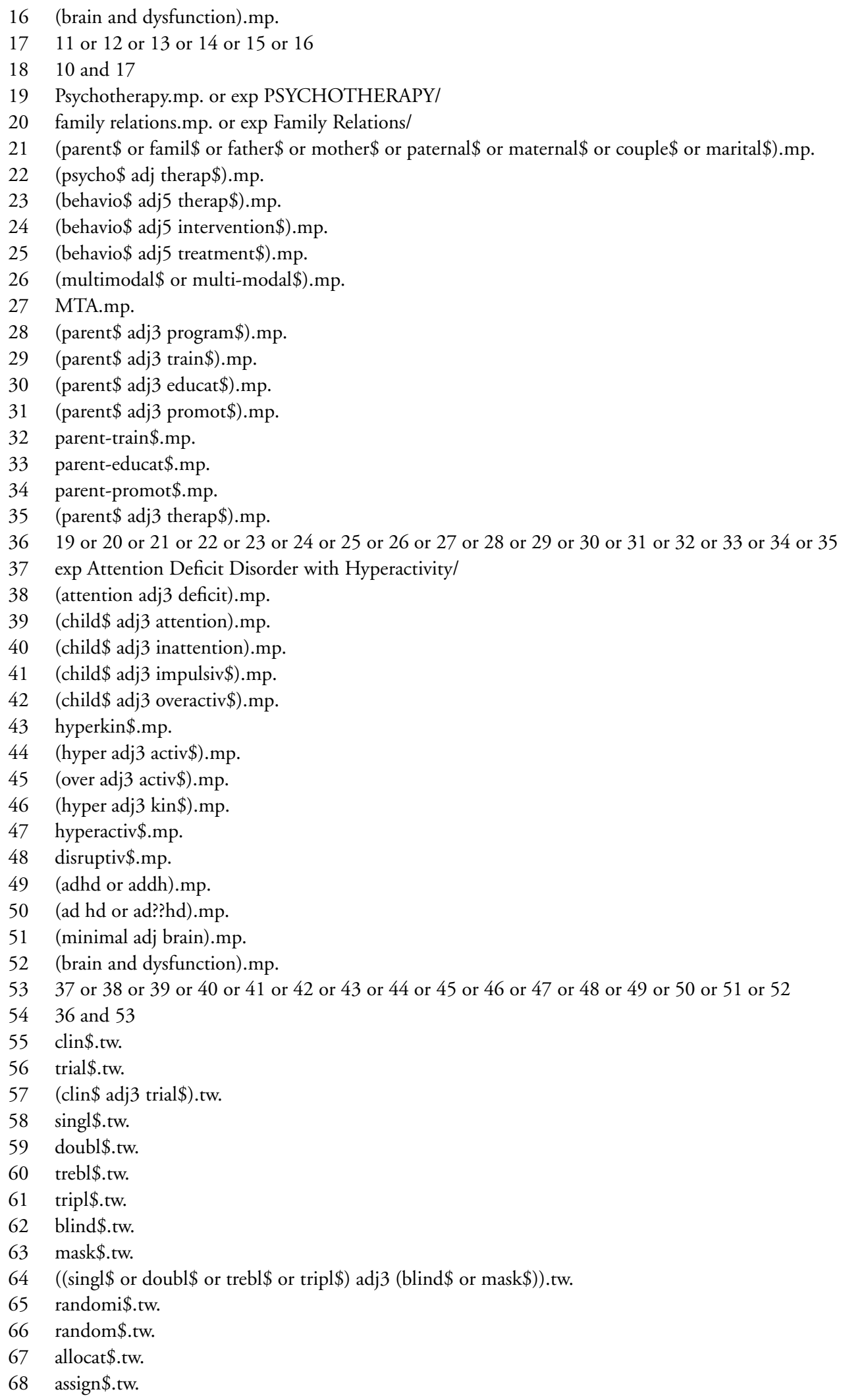

Parent training interventions for Attention Deficit Hyperactivity Disorder (ADHD) in children aged 5 to 18 years (Review) 
(random\$ adj3 (allocat\$ or assign\$)).tw.

crossover.tw.

70 or 69 or 65 or 64 or 57

exp Randomized Controlled Trial/

exp Double Blind Procedure/

exp Crossover Procedure/

exp Single Blind Procedure/

exp RANDOMIZATION/

72 or 73 or 74 or 75 or 76 or 71

54 and 77

\section{Search strategy for PsycINFO (last searched May Week 3 2009)}

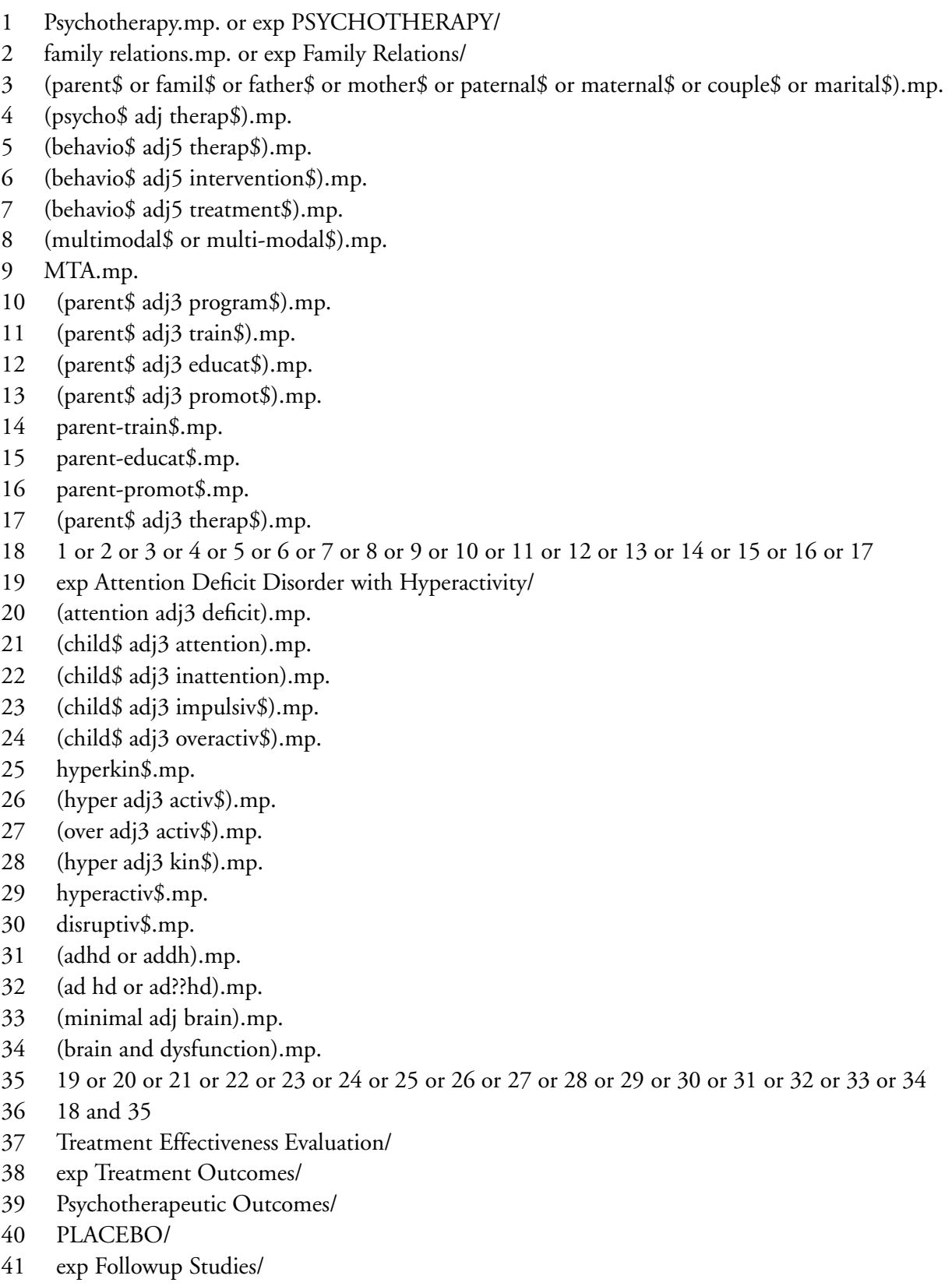

Parent training interventions for Attention Deficit Hyperactivity Disorder (ADHD) in children aged 5 to 18 years (Review)

Copyright (c) 201 I The Cochrane Collaboration. Published by John Wiley \& Sons, Ltd. 
42 placebo $\$ . t w$.

43 random\$.tw.

44 comparative stud\$.tw.

45 randomi\# ed controlled trial\$.tw.

46 (clinical adj3 trial\$).tw.

47 (research adj3 design).tw.

48 (evaluat\$ adj3 stud\$).tw.

49 (prospectiv\$ adj3 stud\$).tw.

50 ((singl\$ or doubl $\$$ or trebl $\$$ or tripl $\$$ ) adj3 (blind $\$$ or mask $\$)$ ).tw.

51 control\$.tw.

5251 or 43 or 41 or 49 or 48 or 44 or 37 or 42 or 38 or 50 or 46 or 40 or 39 or 47 or 45

$53 \quad 36$ and 52

6. Dissertation Abstracts International was searched through Dissertation Express (http://wwwlib.umi.com/dxweb/gateway) (adhd OR addh OR attention deficit OR minimal brain dysfunction OR hyperkinetic syndrome)

7. metaRegister

(adhd OR addh OR attention deficit OR minimal brain dysfunction OR hyperkinetic syndrome)

\section{Appendix 3. Search strategies used September 2010}

1. Cochrane Central Database of Controlled Trials (CENTRAL) 2010 Issue 3 (searched 14 September 2010)

\#1 MeSH descriptor Psychotherapy explode all trees

\#2 MeSH descriptor Family Relations explode all trees

\#3 (parent* or famil* or father* or mother* or paternal* or maternal* or couple* or marital*)

\#4 (psycho* therap*)

\#5 (behavio* near therap*)

\#6 behavio* near intervention*

\#7 behavio* near treatment*

\#8 multimodal $^{*}$ or multi-modal ${ }^{*}$

\#9 (mta)

\#10 (parent* near program*)

\#11 parent $^{*}$ near train*

\#12 parent* near educat $^{*}$

\#13 parent* near promot*

\#14 parent-train*

\#15 parent-educat*

\#16 parent-promot*

\#17 parent* near therap* $^{*}$

\#18 (\#1 OR \#2 OR \#3 OR \#4 OR \#5 OR \#6 OR \#7 OR \#8 OR \#9 OR \#10 OR \#11 OR \#12 OR \#13 OR \#14 OR \#15 OR \#16

OR\#17)

\#19 MeSH descriptor Attention Deficit and Disruptive Behavior Disorders explode all trees

\#20 attention near deficit

\#21 child* near attention

\#22 child* near inattention

\#23 child* near impulsiv*

\#24 child* near overactiv*

\#25 hyperkin*

\#26 hyper near activ*

\#27over near activ*

\#28 hyper near kin*

\#29 hyperactiv*

\#30 disruptiv*

\#31 adhd or addh

Parent training interventions for Attention Deficit Hyperactivity Disorder (ADHD) in children aged 5 to 18 years (Review)

Copyright (C) 20I I The Cochrane Collaboration. Published by John Wiley \& Sons, Ltd. 
\#32 ad next hd

\#33 minimal next brain

\#34 brain and dysfunction

\#35(\#19 OR \#20 OR \#21 OR \#22 OR \#23 OR \#24 OR \#25 OR \#26 OR \#27 OR \#28 OR \#29 OR \#30 OR \#31 OR \#32 OR\#33

OR \#34)

\#36 (\#18 AND \#35)

2. Search strategy for CINAHL via EBSCOhost (searched 13 September 2010)

S55 S37 and S54

S54 S38 or S39 OR S40 OR S41 OR S42 OR S43 OR S44 OR S45 OR S46 OR S47 OR S48 OR S49 OR S50 OR S51 OR S52

S53 S38 or S39 or $S 40$ or $S 41$ or $S 42$ or $S 43$ or $S 44$ or $S 45$ or $S 46$ or $S 47$ or

$\mathrm{S} 48$ or $\mathrm{S} 49$ or $\mathrm{S} 50$ or $\mathrm{S} 51$ or $\mathrm{S} 52$

S52 allocat* random*

S51 (MH "Quantitative Studies")

S50 (MH "Placebos")

S49 placebo*

S48 random* allocat*

S47 (MH "Random Assignment")

S46 (Randomi?ed control* trial $^{*}$ )

S45 (singl* mask*)

S44 (doubl* mask $\left.^{*}\right)$

S43 (tripl* ${ }^{*}$ mask $\left.^{*}\right)$

S42 (trebl* mask $\left.^{*}\right)$

S41 (trebl* blind* ${ }^{*}$ )

S40 (tripl* blind*)

S39 (doubl* blind*)

S38 (singl* blind*)

S37 S19 and S36 Limiters - Published Date from: 20090501-20100931

S36 S20 or S21 or S22 or S23 or S24 or S25 or S26 or S27 or S28 or S29 or S30 or S31 or S32 or S33 or S34 or S35

S35 (MH "Attention Deficit Hyperactivity Disorder")

S34 (brain and dysfunction)

S33 (minimal brain)

S32 (ad hd or ad??hd)

S31 (adhd or addh)

S30 disruptiv*

S29 hyperactiv*

S28 (hyper n3 kin*)

S27 (over n3 activ*)

S26 (hyper n3 activ*)

S25 hyperkin*

S23 (child* n3 impulsiv*)

S22 (child* $\mathrm{n} 3$ inattention)

S21 (child* $n 3$ attention)

$\mathrm{S} 20$ (attention $\mathrm{n} 3$ deficit)

S19 S18 or S17 or S16 or S15 or S14 or S13 or S12 or S11 or S10 or S9 or S8 or S7 or S6 or S5 or S4 or S3 or S2 or S1

S18 (parent* $^{*} 3$ therap*) $^{*}$

S17 parent-promot*

S16 parent-educat*

S15 parent-train*

S14 (parent* n3 promot*) $^{*}$

S13 (parent* 33 educat $\left.^{*}\right)$

S12 (parent* n3 train*)

S11 (parent* $\mathrm{n} 3$ program*)

S10 (multimodal* or multi-modal*)

Parent training interventions for Attention Deficit Hyperactivity Disorder (ADHD) in children aged 5 to 18 years (Review)

Copyright (C) 20I I The Cochrane Collaboration. Published by John Wiley \& Sons, Ltd. 
S9 (behavio* $\mathrm{n} 5$ treatment*

S8 (behavio* $\mathrm{n} 5$ intervention*

S7 (behavio* $n 5$ therap*)

S6 (psycho* N1 therap*)

S5 parent* or famil* or father* or mother* or paternal* or maternal* or couple* or marital*

S4 (MH "Family Relations+")

S3 family relation*

S2 (MH "Psychotherapy+")

S1 Psychotherapy

\section{Search Strategy for EMBASE: 1980 to 2010 Week 36 (searched 24 September 2010)}

1 (parent\$ adj3 program\$).mp.

2 (parent $\$$ adj3 train\$).mp.

(parent\$ adj3 educat\$).mp.

(parent $\$$ adj3 promot $\$$ ).mp.

parent-program $\$ . m p$.

parent-train $\$ . m p$.

parent-educat $\$ . m p$.

parent-promot\$.mp.

(parent $\$$ adj 3 therap\$).mp.

101 or 2 or 3 or 4 or 5 or 6 or 7 or 8 or 9

11 attention deficit disorder.mp. or exp Attention Deficit Disorder with Hyperactivity/

12 (adhd or addh).mp.

13 (attention and deficit).mp.

14 hyperactiv\$.mp.

15 hyperkin\$.mp.

16 (brain and dysfunction).mp.

1711 or 12 or 13 or 14 or 15 or 16

$18 \quad 10$ and 17

19 Psychotherapy.mp. or exp PSYCHOTHERAPY/

20 family relations.mp. or exp Family Relations/

21 (parent $\$$ or famil $\$$ or father $\$$ or mother $\$$ or paternal\$ or maternal $\$$ or couple $\$$ or marital $\$$ ).mp.

22 (psycho\$ adj therap\$).mp.

23 (behavio\$ adj5 therap\$).mp.

24 (behavio\$ adj5 intervention\$).mp.

25 (behavio\$ adj5 treatment\$).mp.

26 (multimodal\$ or multi-modal\$).mp.

27 MTA.mp.

28 (parent\$ adj3 program\$).mp.

29 (parent $\$$ adj3 train $\$$ ).mp.

30 (parent $\$$ adj 3 educat $\$$ ).mp.

31 (parent\$ adj3 promot\$).mp.)

32 parent-train $\$ . m p$.

33 parent-educat\$.mp.

34 parent-promot\$.mp.

35 (parent $\$$ adj 3 therap\$).mp.

3619 or 20 or 21 or 22 or 23 or 24 or 25 or 26 or 27 or 28 or 29 or 30 or 31 or 32 or 33 or 34 or 35

37 exp Attention Deficit Disorder with Hyperactivity/

38 (attention adj3 deficit).mp.

39 (child $\$$ adj3 attention).mp.

40 (child $\$$ adj3 inattention).mp.

41 (child\$ adj3 impulsiv\$).mp.

42 (child\$ adj3 overactiv\$).mp.

Parent training interventions for Attention Deficit Hyperactivity Disorder (ADHD) in children aged 5 to 18 years (Review)

Copyright (c) 201 I The Cochrane Collaboration. Published by John Wiley \& Sons, Ltd. 


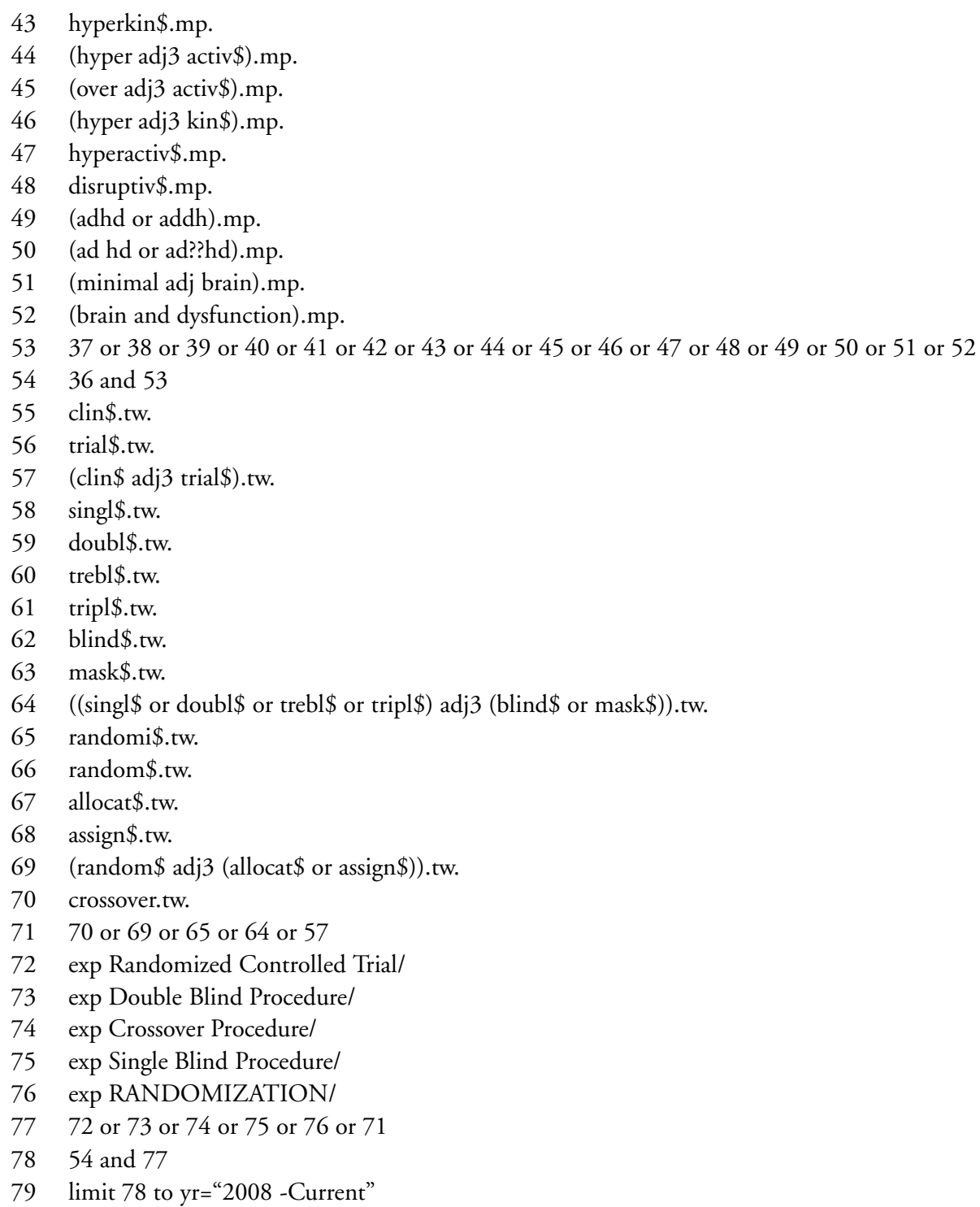

4. Search Strategy for MEDLINE 1950 to September 2010 (searched 14 September 2010)

1 Psychotherapy.mp. or exp PSYCHOTHERAPY/

2 family relations.mp. or exp Family Relations/

3 (parent $\$$ or famil $\$$ or father $\$$ or mother $\$$ or paternal $\$$ or maternal $\$$ or couple $\$$ or marital $\$$ ).mp.

4 (psycho\$ adj therap\$).mp.

5 (behavio\$ adj5 therap\$).mp.

6 (behavio\$ adj5 intervention\$).mp.

7 (behavio\$ adj5 treatment\$).mp.

8 (multimodal $\$$ or multi-modal\$).mp.

9 MTA.mp.

10 (parent $\$$ adj3 program $\$$ ).mp.

11 (parent\$ adj3 train\$).mp.

12 (parent\$ adj3 educat\$).mp.

13 (parent\$ adj3 promot\$).mp.

14 parent-train\$.mp.

15 parent-educat\$.mp.

Parent training interventions for Attention Deficit Hyperactivity Disorder (ADHD) in children aged 5 to 18 years (Review)

Copyright (C) 20I I The Cochrane Collaboration. Published by John Wiley \& Sons, Ltd. 


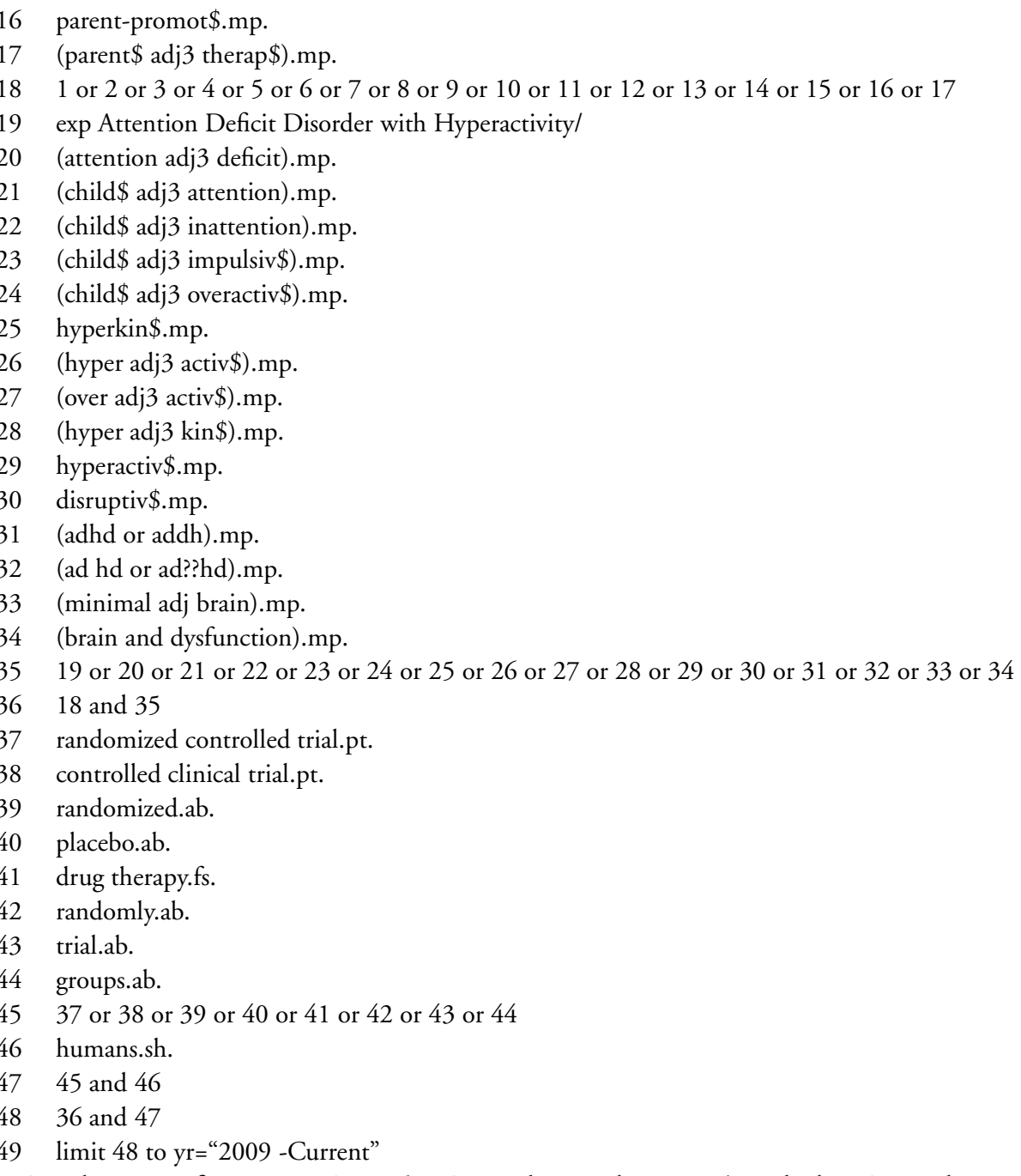

5. Search strategy for PsycINFO 1806 to September Week 12010 (searched 13 September 2010) Psychotherapy.mp. or exp PSYCHOTHERAPY/

family relations.mp. or exp Family Relations/

(parent $\$$ or famil $\$$ or father $\$$ or mother $\$$ or paternal\$ or maternal $\$$ or couple $\$$ or marital $\$$ ).mp.

(psycho\$ adj therap\$).mp.

(behavio\$ adj5 therap\$).mp.

(behavio\$ adj5 intervention\$).mp.

(behavio\$ adj5 treatment\$).mp.

(multimodal\$ or multi-modal\$).mp.

MTA.mp.

10 (parent\$ adj3 program\$).mp.)

11 (parent\$ adj3 train\$).mp.

12 (parent\$ adj3 educat\$).mp.

13 (parent\$ adj3 promot\$).mp.

14 parent-train $\$ . m p$.

15 parent-educat\$.mp.

16 parent-promot\$.mp.

17 (parent\$ adj3 therap\$).mp.

181 or 2 or 3 or 4 or 5 or 6 or 7 or 8 or 9 or 10 or 11 or 12 or 13 or 14 or 15 or 16 or 17

Parent training interventions for Attention Deficit Hyperactivity Disorder (ADHD) in children aged 5 to 18 years (Review) 
9 exp Attention Deficit Disorder with Hyperactivity/

20 (attention adj3 deficit).mp.

21 (child $\$$ adj3 attention).mp.

22 (child $\$$ adj 3 inattention).mp.

23 (child\$ adj3 impulsiv\$).mp.

24 (child\$ adj3 overactiv\$).mp.

25 hyperkin\$.mp.

26 (hyper adj3 activ\$).mp.

27 (over adj3 activ\$).mp.

28 (hyper adj3 kin\$).mp.

29 hyperactiv\$.mp.

30 disruptiv\$.mp.

31 (adhd or addh).mp.

32 (ad hd or ad??hd).mp. [mp=title, abstract, heading word, table of contents, key concepts]

33 (minimal adj brain).mp.

34 (brain and dysfunction).mp.

3519 or 20 or 21 or 22 or 23 or 24 or 25 or 26 or 27 or 28 or 29 or 30 or 31 or 32 or 33 or 34

$36 \quad 18$ and 35 (14495)

37 Treatment Effectiveness Evaluation/

38 exp Treatment Outcomes/

39 Psychotherapeutic Outcomes/

40 PLACEBO/

41 exp Followup Studies/

42 placebo\$.tw.

43 random\$.tw.

44 comparative stud\$.tw.

45 randomi\# ed controlled trial\$.tw.

46 (clinical adj3 trial\$).tw.

47 (research adj3 design).tw.

48 (evaluat\$ adj3 stud\$).tw.

49 (prospectiv\$ adj3 stud\$).tw.

50 ( (singl\$ or doubl\$ or trebl\$ or tripl\$) adj3 (blind $\$$ or mask $\$)$ ).tw.

51 control\$.tw.

5251 or 43 or 41 or 49 or 48 or 44 or 37 or 42 or 38 or 50 or 46 or 40 or 39 or 47 or 45

$53 \quad 36$ and 52

54 limit 53 to $\mathrm{yr}=$ "2009 -Current"

6. Dissertation Abstracts International was searched through Dissertation Express (searched 14 September 2010)

(adhd OR addh OR attention deficit OR minimal brain dysfunction OR hyperkinetic syndrome)

7. The metaRegister of Controlled Trials (mRCT) (searched 14 September 2010)

(adhd OR addh OR attention deficit OR minimal brain dysfunction OR hyperkinetic syndrome)

\section{Appendix 4. Additional methods for future updates}

\section{Measures of treatment effect}

\section{Binary data}

For dichotomous (binary) data, the odds ratio with a $95 \%$ confidence interval will be used to summarise results within each study. The odds ratio is chosen because it has statistical advantages relating to its sampling distribution and its suitability for modelling, and because it is a relative measure and so can be used to combine studies.

Parent training interventions for Attention Deficit Hyperactivity Disorder (ADHD) in children aged 5 to 18 years (Review) 


\section{Categorical data}

Where results are reported in short ordinal scales, the methods of Whitehead and Jones will be used to produce a single odds ratio from each trial (Whitehead 1994). If sufficient detail is not available we will consider analysing such scales as continuous data, after investigating skew and appropriateness.

\section{Unit of analysis issues}

\section{Cluster-randomised trials}

Where trials have used clustered randomisation, we anticipate that study investigators would have presented their results after appropriately controlling for clustering effects (robust standard errors or hierarchical linear models). If it is unclear whether a cluster-randomised trial has used appropriate controls for clustering, the study investigators will be contacted for further information. Where appropriate controls were not used, individual participant data will be requested and re-analysed using multilevel models which control for clustering. Following this, effect sizes and standard errors will be meta-analysed in RevMan using the generic inverse method (RevMan 2008). If appropriate controls were not used and individual participant data is not available, statistical guidance will be sought from the Cochrane Methods Group and external experts as to which method to apply to the published results in an attempt to control for clustering. Our preferred method (if there is insufficient information to control for clustering) will be to perform a sensitivity analysis assuming a variety of intraclass correlations (ICCs) from standard tables or using those from other similar studies in the field. As a last resort, outcome data may be entered into RevMan using individuals as the units of analysis, and then sensitivity analysis will be used to assess the potential biasing effects of inadequately controlled clustered trials (Donner 2001).

\section{Assessment of reporting biases}

To investigate the possibility of reporting biases, including publication bias, funnel plots will be drawn (Egger 1997; Sterne 2001; Deeks 2005;). In the event of asymmetry, the reviewers will seek input from methodologists, including the Cochrane and Campbell Collaboration Methods Groups, on appropriate analyses, given concerns raised in Chapter 10.4.2 of the Cochrane Handbook (Sterne 2008).

We plan also to address the issue of selective outcome reporting by checking against the included studies' protocols if possible (within trial registries, conference proceedings, etc.), and by internal evidence within the published studies.

\section{Data synthesis}

\section{Subgroup analysis and investigation of heterogeneity}

Large numbers of subgroups may lead to misleading conclusions and are best kept to a minimum (Yusuf 1991; Oxman 1992). If possible, this review will include separate effect estimates for the following subgroups:

- children receiving concomitant drug treatment;

- parent training programmes that are group based versus individual or couple based programmes;

- parent training programmes that involve a teacher component.

\section{Sensitivity analysis}

Sensitivity analyses will be carried out:

- to consider the impact of differing ratings on pre-specified 'Risk of bias' criteria, especially to assess whether results are sensitive to restricting the analyses to studies judged to be at low risk of bias with regard to: (a) selection bias (associated with sequence generation or allocation concealment); (b) only studies with low risk of attrition bias (associated with completeness of data);

- to analyse the differential impact of true versus 'quasi' randomised studies;

- to compare studies in which fidelity testing was undertaken compared to those where it was not done.

In addition, studies in which participants are analysed as members of the groups to which they were originally assigned (intention-totreat analysis), studies that include only those participants who were willing or able to provide data (available-case analysis), and studies

Parent training interventions for Attention Deficit Hyperactivity Disorder (ADHD) in children aged 5 to 18 years (Review) 
that analyse participants who adhered to the study's design (per-protocol analysis; Higgins 2005) will be analysed separately. Studies in which the reasons for excluding participants from analyses can not be determined from relevant reports or through contact with the authors will be considered with per-protocol analyses.

\section{H I S T O R Y}

Protocol first published: Issue 2, 2001

Review first published: Issue 12, 2011

\begin{tabular}{l|l|l}
\hline Date & Event & Description \\
\hline 1 May 2009 & New citation required and major changes & $\begin{array}{l}\text { This protocol has been substantially updated since first } \\
\text { publication in 2001 and has been co-registered within } \\
\text { the Campbell Collaboration }\end{array}$ \\
\hline 9 September 2008 & Amended & Hannah Jones added to authorline \\
\hline 9 September 2008 & Amended & Converted to new review format. \\
\hline 4 July 2007 & Amended & $\begin{array}{l}\text { Camilla Thorgaard added to authorline; Sima Pindoria } \\
\text { and Carol Joughin removed }\end{array}$ \\
\hline
\end{tabular}

\section{CONTRIBUTIONS OFAUTHORS}

Morris Zwi (MZ) conceived and designed the original review question and wrote the background of the protocol with assistance from Ann York (AY). Jane Dennis (JD), Hannah Jones (HJ) and Camilla Thorgaard (CT) contributed to the refining of the inclusion criteria. $\mathrm{MZ}, \mathrm{JD}$ and $\mathrm{HJ}$ wrote the Methods sections.

Results were vetted in pairs by MZ, JD, HJ and AY. Studies were assessed for eligibility and data were extracted and entered into RevMan 5.0 in pairs by MZ, JD, HJ and CT. The final review was written by MZ, JD, HJ and AY.

\section{DECLARATIONSOF INTEREST}

Morris Zwi - this research was facilitated by the receipt of a PPP Mid-Career Award which funded a locum 2.5 days per week for one year to allow completion of a diploma in Systematic Reviews methodology at the Systematic Reviews Training Unit, UCL. The

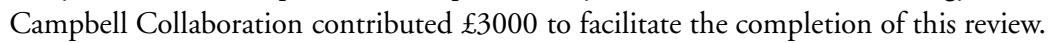

Hannah Jones - none known.

Camilla Thorgaard - none known.

Ann York - none known.

Jane A Dennis - none known. 


\section{SOURCES OF SUPPORT}

\section{Internal sources}

- University of Bristol, UK.

\section{External sources}

- PPP Healthcare Medical Trust "Mid-Career Awards" grant, UK.

- SFI Campbell, The Danish National Centre for Social Research, Denmark.

\section{DIFFERENCESBETWEEN PROTOCOLANDREVIEW}

- In response to feedback from an external peer referee, we acknowledge that parent training in ADHD is mainly aimed at reducing $\mathrm{ADHD}$ symptoms and associated behaviour problems, so academic achievement would generally be seen to be a secondary rather than a primary outcome. We have changed the list of primary and secondary outcomes to reflect this.

- We updated the form of the search flow diagram from the QUOROM to the PRISMA statement.

- We deleted a proposed subgroup analysis listed in our protocol, regarding 'children with disruptive behaviour disorders where ADHD is specified as a subgroup', because we realised that it was in direct conflict with one of our inclusion criteria, namely that ADHD should be the focus of any included study. See also 'Discussion' for why this decision was taken (namely that studies wherein ADHD is not the prime focus tend inevitably to have excluded 'typical' ADHD children).

- The section on 'Unit of analysis' issues (now published in Appendix 4, Additional methods for future updates) contains a slight alteration to plans for the analysis of cluster-randomised controlled trials.

- The section on 'Sensitivity analysis' issues (now published in Appendix 4, Additional methods for future updates) contains a plan to consider the impact of differing ratings on pre-specified 'Risk of bias' criteria within sensitivity analyses, and plans to assess whether results are sensitive to restricting the analyses to studies judged to be at low risk of bias with regard to: (a) selection bias (associated with sequence generation or allocation concealment); (b) only studies with low risk of attrition bias (associated with completeness of data).

\section{NOTES}

This review is co-registered within The Campbell Collaboration (www.campbellcollaboration.org).

\section{N D EX TERMS}

\section{Medical Subject Headings (MeSH)}

*Parenting; Attention Deficit Disorder with Hyperactivity [psychology; * rehabilitation]; Child Behavior Disorders [psychology; rehabilitation]; Parents [*education; psychology]; Randomized Controlled Trials as Topic; Stress, Psychological [therapy] 


\section{MeSH check words}

Adolescent; Child; Child, Preschool; Female; Humans; Male 